ADDENDUM

DOE/ER/40 444--4-Add.

DE9 2006451

\title{
Studies in Medium Energy Physics
}

\author{
Progress Report \\ and \\ Continuation Proposal
}

April 1, 1991 - March 31, 1992

\begin{abstract}
A. Green, G. W. Hoffmann, J. McDonough, M. J. Purcell, R. L. Ray, D. E. Read, and S. D. Worm

Department of Physics

The University of Texas at Austin

Austin, Texas 78712
\end{abstract}

December 1991

PREPARED FOR THE U.S. DEPARTMENT OF ENERGY

UNDER GRANT NUMBER DE-FG05-88ER40444

1

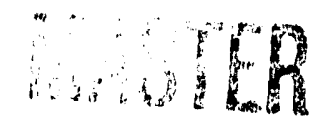

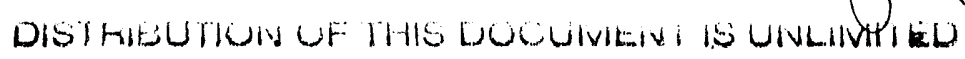


$\mathrm{DE} / \mathrm{ER} / 40444-4$

\title{
Studies in Medium Energy Physics
}

\author{
Progress Report \\ and \\ Continuation Proposal
}

April 1, 1991 - March 31, 1992

\author{
A. Green, G. W. Hoffmann, J. McDonough, \\ M. J. Purcell, R. L. Ray, D. E. Read, and S. D. Worm \\ Department of Physics \\ The University of Texas at Austin \\ Austin, Texas 78712
}

December 1991

PREPARED FOR THE U.S. DEPARTMENT OF ENERGY UNDER GRANT NUMBER DE-FG05-88ER40444 


\section{Preface}

This document constitutes the 1991 - 1992 technical progress report and continuation proposal for Special Research Grant DE-FG05-88ER40444 from the U. S. Department of Energy to support research in medium energy physics by researchers at The University of Texas at Austin.

The data, calculations, and other information presented here represent work in progress, are preliminary, and should not be quoted without the authors' permission.

Further information relating to the technical portions of this document may be obtained from Dr. G. W. Hoffmann, Department of Physics, The University of Texas at Austin, Austin, Texas 78712, telephone (512) 471-1769 or Bitnet: "HOFFMANN@UTAPHY".

\section{DISCLAIMER}

This report was prepared as an account of work sponsored by an agency of the United States Government. Neither the United States Government nor any agency thereof, nor any of their employees, makes any warranty, express or implied, or assumes any legal liability or responsibility for the accuracy, completeness, or usefulness of any information, apparatus, product, or process disclosed, or represents that its use would not infringe privately owned rights. Reference herein to any specific commercia! product, process, or service by trade name, trademark, manufacturer, or otherwise does not necessarily constitute or imply its endorsemcial, recommendation, or favoring by the United States Government or any agency thereof. The views and opinions of authors expressed herein do not necessarily state or reflect those of the United States Government or any agency thereof. 


\begin{abstract}
This document constitutes the (1991 - 1992) technical progress report and continuation proposal for the ongoing medium energy nuclear physics research program supported by the U. S. Department of Energy through special Research Grant DE-FG05-88ER40444.

The experiments discussed are conducted at the Los Alamos National Laboratory's (LANL) Clinton P. Anderson Meson Physics Facility (LAMPF) and the Alternating Gradient Synchrotron (AGS) facility of the Brookhaven National Laboratory (BNL).

The overall motivation for the work discussed in this document is driven by three main objectives: (1) provide hadron-nucleon and hadron-nucleus scattering data which serve to facilitate the study of effective two-body interactions, test (and possibly determine) nuclear structure, and help study reaction mechanisms and dynamics; (2) provide unique, first-of-a-kind "exploratory" hadron-nucleus scattering data in the hope that such data will lead to discovery of new phenomena and new physics; and (3) perform precision tests of fundamental interactions, such as rare decay searches, whose observation would imply fundamental new physics.
\end{abstract}




\section{Contents}

1 Introduction 5

2 Continuing Projects $\quad 7$

2.1 EXP955: Polarized Pruton Elastic Scattering from Polarized ${ }^{13} \mathrm{C}$ and ${ }^{1} \mathrm{H} \ldots \ldots \ldots 7 \ldots \ldots \ldots \ldots$

2.2 EXP1079: $p+$ A Precision Elastic Forward Angle Cross Sections . 9

2.3 EXP1131: Measurement of Polarization Transfer for $800 \mathrm{MeV}$ Inclusive Proton Scattering at the MRS . . . . . . . . . . . . . 14

2.4 Polarized Nuclear Target Project . . . . . . . . . . . . . . . 16

2.5 BNL EXP791/871: Search For Very Rare $K_{L}$ Decays . . . . . . . . 19

3 New Experimental Projects $4 \mathbf{4 1}$

3.1 EXP1040: Spin Variable Measurements of the $(p, n)$ Inclusive Reaction on Several Nuclei ......... . . . . . . . . . 41

3.2 EXP1097: Single Pion Production in $n p$ Scattering . . . . . . . . 47

3.3 EXP1145: Measurements of Complete Spin Observables in the Quasifree Region for Medium Energy $\left(p, p^{\prime}\right) \ldots \ldots 52$

3.4 EXP1178: Polarization Asymmetry Measurements for the ${ }^{1} \overrightarrow{\mathrm{H}}\left(\pi^{-}, \pi^{0}\right) n$ Reaction Between 45 and $190 \mathrm{MeV}$. . . . . . . . . . . . 54

3.5 EXP1205: Precision Measurement of $D_{N N}$ for ${ }^{13} \mathrm{C}(\vec{p}, \vec{p})$ at $500 \mathrm{MeV} 57$

3.6 EXP1206: Polarized Proton Elastic Scattering from :-olarized ${ }^{6} \mathrm{Li}$ at $500 \mathrm{MeV} \ldots \ldots . \ldots 63$

4 Theoretical Work

4.1 Review of Nonrelativistic and Relativistic Descriptions of ProtonNucleus Scattering . . . . . . . . . . . . . . . . 72

4.2 Nuclear Structure and Medium Effects in $\vec{p}+{ }^{13} \overrightarrow{\mathrm{C}}$ Elastic Scattering 82

4.3 Proton Elastic Scattering from Polarized ${ }^{3} \mathrm{He} \ldots \ldots . . . . . . .88$

4.4 New Theoretical Work ... . . . . . . . . . . . 88

5 Publications (1990 - 1991) 91

5.1 Journal and Conference Proceedings in Print . . . . . . . . . . . . 91

5.2 Journal Articles Submitted for Publication . . . . . . . . . . . . 93

5.3 EXP791/871 KL-Memos (Internal Publications) . . . . . . . . . 93

5.4 Abstracts ........................... 94 


\section{Introduction}

This document is a progress report for 1991 - 1992 and continuation proposal for research projects in medium energy nuclear physics (MEP) supported by the $U$. S. Department of Energy's Three-Year Grant No. DE-FG05-88ER40444 with the University of Texas at Austin for the 1991 - 1994 period. University of Texas personnel associated with these projects for the 1991-1992 period (not inclusive) are G. W. Hoffmann (Professor); R. L. Ray (Research Scientist), J. E. McDonough and A. Green (post-doctoral fellows); M. J. Purcell, D. E. Read, and S. D. Worm (Graduate Students).

Our research program in Medium Energy Physics (MEP) has three major areas of thrust, as described in the paragraphs below. The specific work carried out during 1991 - 1992 and to be done for the remainder of the 1991-1994 period, as discussed in detail in this document, fits into this overall scheme. The work to be done consists of continuing projects (started before 1991) as well as new initiatives which span 1991-1994 and beyond.

One of the sustained goals of our research program has been to provide precision medium energy hadron-nucleon and hadron-nucleus scattering data, as well as pragmatic, state-of-the-art analyses, which serve to facilitate the systematic study of effective two-body interactions, to test (and possibly determine) nuclear structure, and to help study reaction mechanisms and dynamics. Considerable and steady progress has been made towards accomplishing these goals. For the $p$ + nucleus case, it is fair to say that, today, reasonably quantitative descriptions of a vast amount of data for energies between 100-1000 MeV, and nuclei spanning the periodic table, are obtained using a variety of microscopic models [both relativistic and nonrelativistic] which are built up from first principles. We have recently completed a lengthy review article for Physics Reports which describes this research effort. This program of research has been very fruitful, and its success is due in part to the fact that over the years experiment and theory have merged to play very complementary roles in the program. We have provided a considerable quantity of the $500-800 \mathrm{MeV} p+$ nucleus and $p+$ nucleon data that are part of the data base used in the national program; these data consist of differential cross sections, analyzing powers, and spin-rotation-depolarization observables. We were also instrumental in formulating and developing the microscopic theoretical models used in the analyses. Some of the work done during the 1991-1992 period was the natural continuation of this very successful program. One example of an experiment in this area which we have made progress on this year is our precision (1\%) forward-angle proton-nucleus elastic scattering experiment which will provide cross section data in the Coulomb-nuclear interference region; the relativistic and nonrelativistic models give cross sections in this region whose differences are typically $15-25 \%$. 
Another fruitful part of our research program that has shown itself invaluable over the years to progress in medium energy hadron-nucleus physics consists of unique, first-of-a-kind, "exploratory" experiments which are done to search for new, unexpected phenomena and new physics, and which provide data to stimulate further theorntical work. A good example is our $500 \mathrm{MeV} \vec{p}+{ }^{13} \overrightarrow{\mathrm{C}}$ experiment which used, for the first time, polarized nuclear targets. Such experiments add a new spin degree of freedom to the scattering problem. Experiments involving polarized nuclear targets will continue to be an important part of our research program. We are presently constructing a state-of-the-art polarized target which uses a superconducting magnet and dilution refrigerator. We also have another approved experiment at LAMPF to do polarized proton elastic scattering from a polarized nuclear target.

Finally, concerning the overall picture of our program, we note that in more recent years, it has been realized that the study of fundamental interactions forms a very important and vital branch of research in medium energy physics. Measurements performed in the past few years make it clear that any possible incompleteness of our present understanding of fundamental interactions must originate with physics at very large mass scales. Rare decay physics probes some of these higher mass scales. Facilities such as The Los Alamos National Laboratory's Clinton P. Anderson Meson Physics Facility and the Brookhaven National Laboratory's Alternating Gradient Synchrotron (AGS) facility can produce copious quantities of a wide variety of unstable particles whose decay modes can be examined. Recently, we broadened our research program to include an experiment at the AGS which searched for the forbidden decay $K_{L}^{0} \rightarrow \mu e$ and the suppressed decay $K_{L}^{0} \rightarrow e e$. One result of this experiment is the smallest upper limit to date for these unobserved decays. A new experiment (with more than an order of magnitude improvement in sensitivity) has been initiated. Its purpose is to improve the single event sensitivity for $K_{L}^{0} \rightarrow \mu e$ to less than $1 \times 10^{-12}$, and if no events are observed, will set an upper limit of about $2 \times 10^{-12}$. The new experiment should also see a few $K_{L}^{0} \rightarrow e e$ if this process occurs at the expected level. The new experiment will also observe about $10,000 K_{L}^{0} \rightarrow \mu \mu$ decays.

Our resources are split roughly 50-50 between the efforts at LANL and BNL. We are heavily involved in the new $K_{L}^{0}$ rare decay experiment at BNL and typically carry out 1-2 experiments with Texas spokesmen per year at LAMPF. Work on the polarized target/test facility project is also being vigorously pursued. We also contribute to other LAMPF experiments for which we are not spokesmen, but participants, as time permits.

Our group has authored 12 refereed journal articles, 4 published conference proceedings, 7 BNL technical reports, and 5 abstracts during the 1991-1992 research period.

The material contained in this document summarizes the highlights of our 1991-1992 research program. 


\section{Continuing Projects}

All projects in this section, apart from LAMPF EXP1131, have been active this year and will continue to be so during the coming year and beyond. EXP1131, although initiated in the Summer of 1990, saw no experimental activity during 19911992, owing to the shutdown of MRS due to radiation safety concerns. The data taking phase of EXP955 was completed some time ago; yet important $\vec{p}+\vec{p}$ elastic $A_{\text {oonn }}$ data taken during that experiment remain to be finalized and published. EXP1079, the Polarized Target Project, and design-development-preparation for BNL E871 received most of our experimental effort this year and are projected to constitute a large fraction of our effort next year.

\subsection{EXP955: Polarized Proton Elastic Scattering from Po- larized ${ }^{13} \mathrm{C}$ and ${ }^{1} \mathrm{H}$}

(G. W. Hoffmann, M. L. Barlett, W. Kielhorn, G. Pauletta, M. Purcell, L. Ray; LANL; NMSU; Minnesota; ASU; OSU; IBM;

G. W. Hoffmann, R. L. Ray, M. L. Barlett, J. Jarmer, spokesmen)

Summary: $500 \mathrm{MeV} \vec{p}+\vec{p}$ elastic $A_{\text {oonn }}$ data were taken during the $\vec{p}+{ }^{13} \overrightarrow{\mathrm{C}}$ experiment. These very high precision, forward-angle data remain to be reduced to final form and published.

The main objective of this experiment was to provide the first $A_{\text {ooon }}$ and $A_{\text {oonn }}$ data for polarized proton scattering from a polarized nuclear target. The results for $\vec{p}+{ }^{13} \overrightarrow{\mathrm{C}}$ elastic scattering at $500 \mathrm{MeV}$ have now been published. ${ }^{1}$

The target material was ethylene glycol, ${ }^{13} \mathrm{C}_{2} \mathrm{H}_{6} \mathrm{O}_{2}$. Since the ${ }^{1} \mathrm{H}$ in the target was also polarized, at each angle setting where $\vec{p}+{ }^{13} \overrightarrow{\mathrm{C}}$ elastic data were taken, the High Resolution Spectrometer (HRS) fields were lowered and data were taken for $\vec{p}+\vec{p}$ elastic scattering. The intent was that these nucleon-nucleon data would serve to verify the procedures used to extract $A_{\text {oonn }}$ and $A_{\text {ooon }}$ in the polarized nucleus experiment.

Surprisingly, however, we find that there are no high quality measurements of $A_{\text {oonn }}$ for $\vec{p}+\vec{p}$ elastic scattering at energies near $500 \mathrm{MeV}$. An examination of Arndt's data base reveals that errors in the experimental data are typically in excess of \pm 0.06 for center-of-momentum scattering angles less than 45 degrees. Our preliminary data are shcwn in Fig. 1. The errors are typically $\pm 0.01-0.02$. The curve is from Arndt's phase shift solution SP89. It is seen that the data and the SP89 solution do not agree quantitatively, both in magnitude and in position of the maximum in $A_{\text {oonn }}$. Our new, high quality data should serve useful for fine 
tuning this phase shift solution.

We hope to finalize and publish these new nucleon-nucleon elastic data in the coming year.

\section{References}

[1] G. W. Hoffmann, et al., Phys. Rev. Lett. 65, 3096 (1990).

\section{Figure Caption}

Figure 1: $A_{\text {oonn }}$ for $\vec{p}+\vec{p}$ elastic scattering at $500 \mathrm{MeV}$. The curve is from phase shift solution SP89 (Arndt).

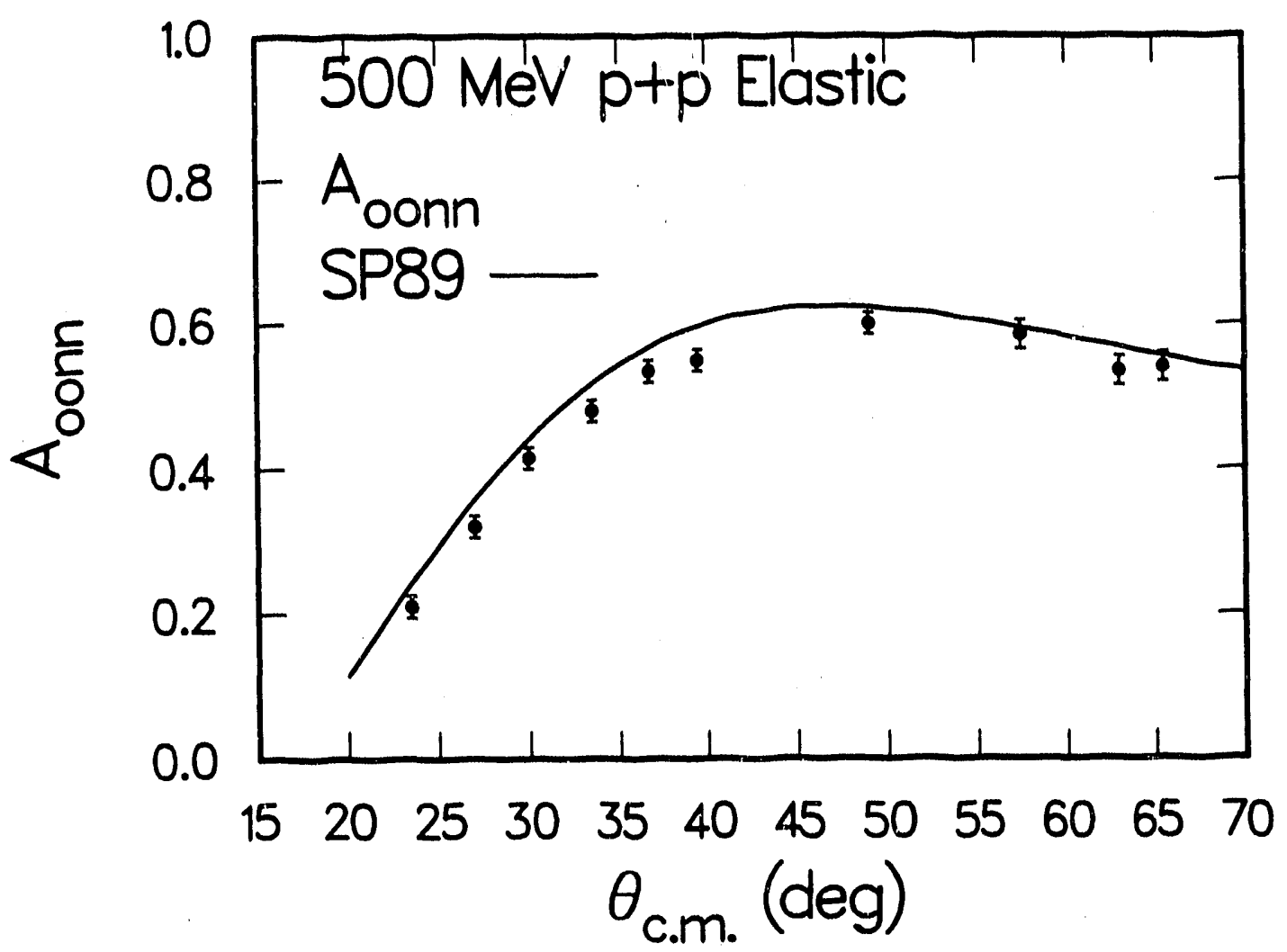




\title{
2.2 EXP1079: $p+$ A Precision Elastic Forward Angle Cross Sections
}

\author{
(A. Green, G. W. Hoffmann, L. Ray, D. Read, S. Worm; LANL; \\ G. W. Hoffmann, J. F. Amann, spokesmen)
}

Summary: The development and apparatus construction phase of this $1 \%$ absolute differential cross section experiment for $p+A$ elastic scattering is nearly complete, and data will be taken in Summer 1992.

A comparison of observables predicted by scattering models which use either relativistic (RIA) or nonrelativistic (NRIA) dynamics indicates ${ }^{1}$ significant differences in the magnitudes of the $500-800 \mathrm{MeV} \mathrm{p}+{ }^{16} \mathrm{O},{ }^{40} \mathrm{Ca},{ }^{208} \mathrm{~Pb}$ differential cross sections in the forward-angle, Coulomb-nuclear interference region (see Fig. 1). At $500 \mathrm{MeV}$ some of the differences are $15-25 \%$. This result is stable with regard to uncertainties in the theoretical calculations $( \pm 2-6 \%)$ including medium effects, and suggests that techniques be developed to perform the required experiment to test the various models. It has also been pointed out that precise cross section data in the Coulomb-nuclear region would set important constraints on relativistic models of the virtual pair process and the scalar densiities of the target nucleus. Once developed, the techniques could also be used to renormalize to the level of $1 \%$ the vast quantity of $p+A$ elastic scattering data that has been taken at LAMPF over the past 15 years.

The first phase of EXP1079 has been to develop the techniques required for a $1 \%$ experiment. Of particular importance are accurate integrated beam flux determination $(0.5 \%)$, precise polar scattering angle determination $\left(0.001^{\circ}\right)$, and accurate solid angle determination $(0.1 \%)$.

During our first two development runs we commissioned the new HRS Faraday cup, a high gain (xenon-ethane $600 \mathrm{~mm} \mathrm{Hg}$ ) ion chamber, a beam counting scintillator telescope, and the new Line C inductive pickup coil (LCCM). We demonstrated that with care there is sufficient overlap in the beam intensity regions over which these devices operate that together they can redundantly determine beam current over the dynamic range of $10^{4} / \mathrm{s}$ to several $\mathrm{nA}$ with better than $1 \%$ absolute accuracy.

We demonstrated that the solid angle can be determined to the $0.1 \%$ level using slits of aperture $10 \%$ of the HRS acceptance. Slit edge scattering was demonstrated to have negligible effect on the solid angle defined by the aperture (note that medium energy protons that go through the slit material loose about $10 \mathrm{MeV}$ so that they are shifted in the missing-mass spectrum generated by the HRS; nominal HRS resolution is $100 \mathrm{keV}$ ).

We designed beam scanning scintillators which step through the beam to accurately provide the integral $\int_{0}^{x} I(x) d x$, where $I(x)$ is the beam intensity at transverse 
position $x$. These scintillators are $1 \mathrm{in}$. by $1 \mathrm{in}$. by $1 \mathrm{~mm}$, and are coupled to EMI9813B photomultipliers. Specially designed tube bases which operate in vacuum allow these tubes to operate between $200 \mathrm{~V}$ and $2000 \mathrm{~V}$, providing an effective gain dynamic range of $10^{9}$. The system is designed for beams of a few $\mathrm{kHz}$ up to about $10 \mathrm{GHz}$. The beam scanners are mounted on linear slides and the positions are determined to 0.0002 in. using Heidenhain optical encoders. These linear encoders measure light that is transmitted through a glass diffraction grating (see Fig. 2). When the grating is moved relative to the scanning unit, the lines and spaces of the scale alternately coincide with those of the index gratings. The corresponding fluctuations of the light are sensed by photocells, which generate two sinusoidal output signals, which are phase-shifted 90 degrees relative to each other. These signals are then interpolated and converted to square waves with a Schmitt trigger. The 90 degree phase shift between the signals allows for four signal edges which can be used to determine the direction of the motion.

A test run was made in Summer 1991 to evaluate the performance of the beam scanners. A Line C (HRS) pencil beam was prepared using the Line $\mathrm{C}$ collimators. Fig. 3 shows the results of a typical beam scan made with a horizontal scanner in the HRS scattering chamber. The top curve is the integrated light output (relative) from a horizontal scanner as it is stepped through the beam. The bottom curve is the derivative of the top curve. In both top and bottom figures the horizontal scale is in inches. For the particular scan shown here, the FWHM of the beam is about $0.010 \mathrm{in}$. and the centroid position was determined to $0.001 \mathrm{in}$. This beam profile is typical of that required for the forward angle part of the exeriment.

A considerable amount of effort this year went into the design and mechanical construction of the actual EXP1079 apparatus to be used for the production data. One man-year of machine shop work has been provided for this project by the Physics Department machine shop. The system, whose components are all remotely moveable via computer control, are each mounted on an aluminum tooling plate that fits inside the HRS scattering chamber. The components consist of 4 beam scanners (an $x y$ pair before and after the target, separated by about 30 in.), 2 high gain $(\times 1000)$ ion chambers, 3 beam counting scintillators (for low intensity beams), a new target ladder, and a moveable, precision slit system made of tool steel with tapered rectangular apertures. The reference positions of the beam scanners, the target, and the slit apertures are to be determined using an elaborate aligning procedure to an accuracy of about $0.001 \mathrm{in}$. The optical encoders then will give relative positions to an accuracy of $0.0002 \mathrm{in}$. Much of this apparatus was commissioned during the Summer 1991 development run.

A final set of modifications and refinements is currently underway. We expect to commission the entire apparatus in Summer 1992 and take the initial production data at the same time. 


\section{References}

[1] L. Ray, G. W. Hoffmann, M. L. Barlett, and N. Ottenstein, Phys. Rev. C 37, 224 (1988).

\section{Figure Captions}

Figure 1: Uncertainty bands for the forward angle differential cross sections for $500 \mathrm{MeV} p+{ }^{40} \mathrm{Ca}$ and ${ }^{208} \mathrm{~Pb}$ from the standard NRIA (shaded bands between dashed curves) and RIA (shaded bands between the solid curves) models (from Ref. 1).

Figure 2: Schematic of Heidenhain optical encoder.

Figure 3: Horizontal profile of LAMPF Line $\mathrm{C}$ pencil beam at HRS scattering chamber as determined using EXP1079 beam scanners.
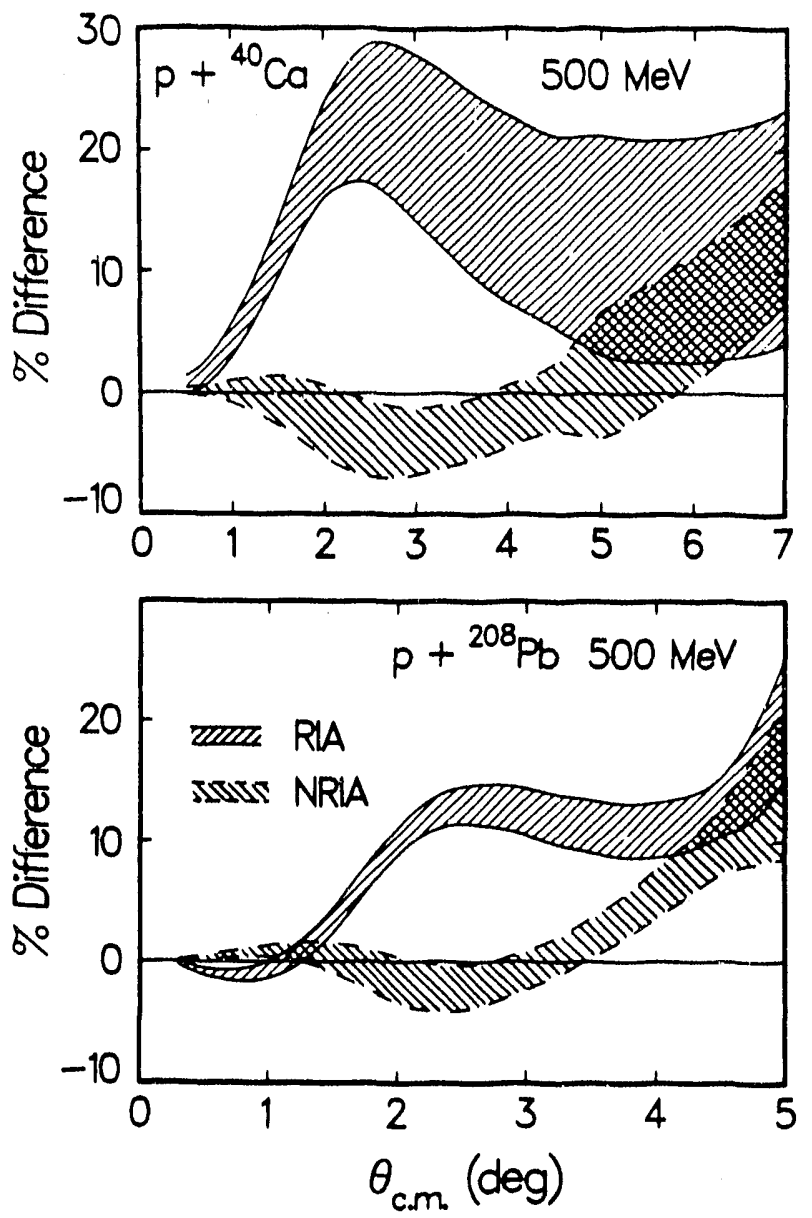

Figure 1 


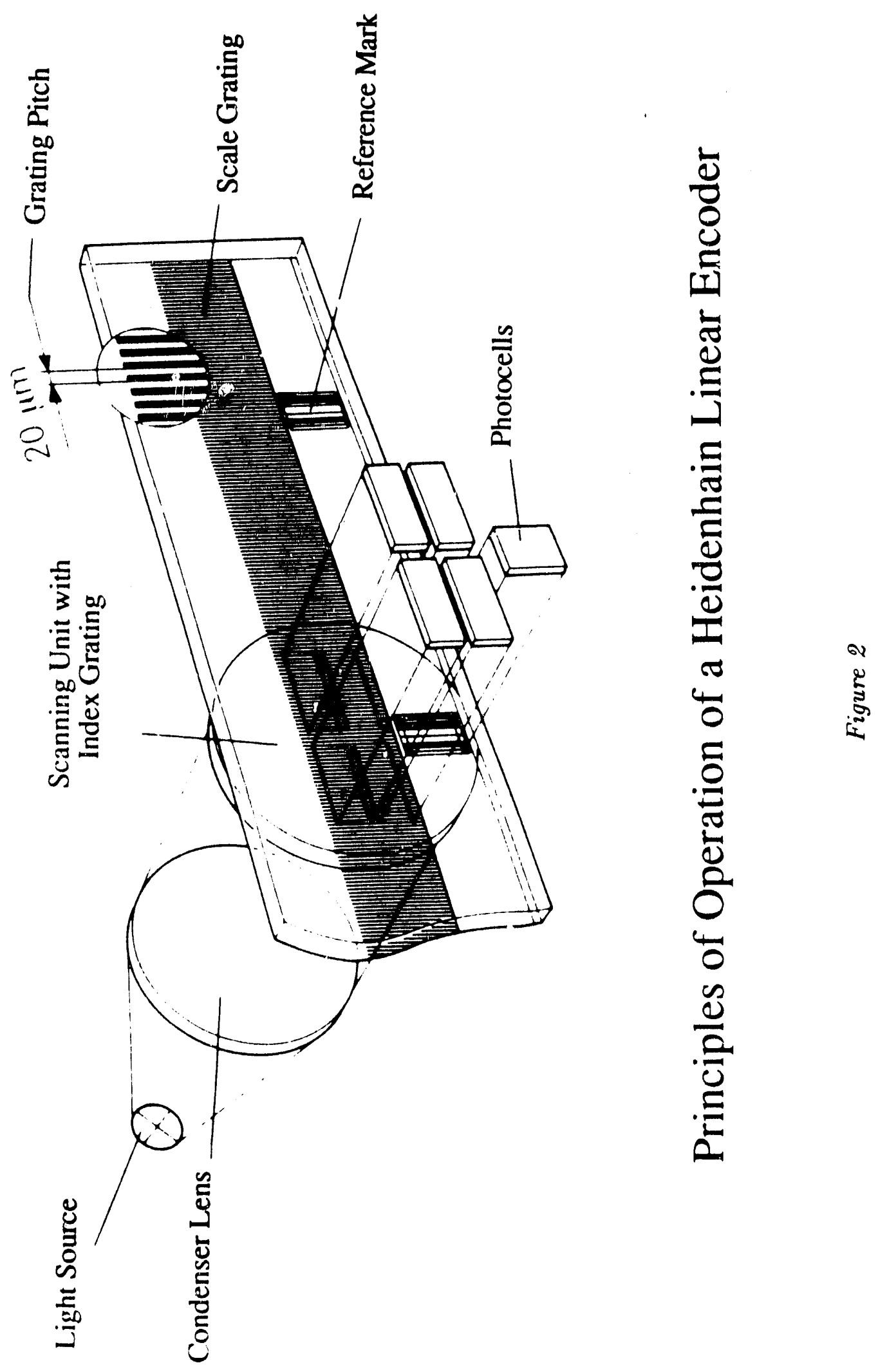



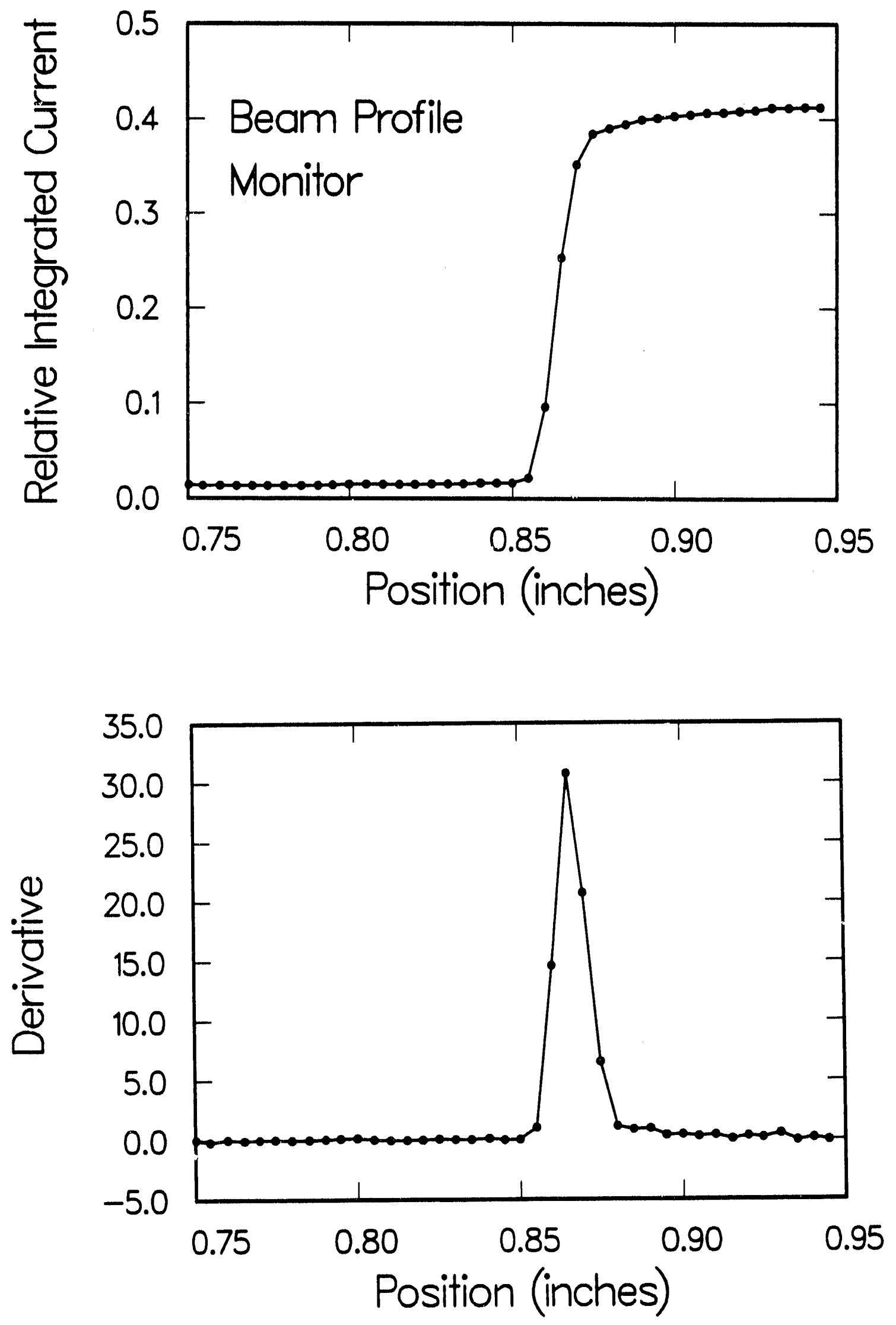

Figure 3 


\title{
2.3 EXP1131: Measurement of Polarization Transfer for $800 \mathrm{MeV}$ Inclusive Proton Scattering at the MRS
}

\author{
(G. Hoffmann, D. Read, S. Worm; Rutgers; LANL; Georgia; Orsay; \\ C. Glashausser, G. W. Hoffmann, K. Jones, spokesmen)
}

Summary: This experiment measures $D_{i j}$ spin-observables for $800 \mathrm{MeV}\left(p, p^{\prime}\right)$ in the $\Delta(1232)$ region. The experiment was used to commission the MRS and is currently on hold pending radiation safety concerns at LAMPF.

The high-excitation energy response of the nucleus to medium energy probes has been the subject of considerable interest. A fundamental question is whether the nuclear response in this region is close to that of a noniriteracting gas of fermions or whether there are specific many-body effects that are important even at highexcitation energies. If a Fermi gas picture is valid and distortions and Fermimotion effects are small, then spin-observables such as the analyzing power $A_{y}$ or the polarization-transfer coefficients $D_{i j}$ for inelastic proton scattering should be very close to the values for nucleon-nucleon $(N N)$ scattering. Deviations from the Fermi gas predictions could result, for example, from nuclear structure effects such as dynamical correlations which persist even at high-excitation energy. Another medium effect is suggested by applications of the Dirac equation to the description of nuclear structure and reactions. Calculations indicate that many of these effects are most easily seen in the behavior of spin observables in scattering experiments.

EXP626, "Polarization Transfer for Inclusive Proton Nucleus Inelastic Scattering at $800 \mathrm{MeV}$," was done with the high resolution spectrometer (HRS). That experiment ${ }^{1}$ measured some of the spin-observables for the high-excitation $(\Delta)$ region. However, because of time limitations and intrinsic constraints from spin precession in the HRS dipoles, the data have relatively large statistical errors and have a number of gaps for various observables at different outgoing proton energies. These data do not have the statistical accuracy to distinguish small but possibly significant effects of the nuclear medium on various spin observables, and the gaps and poor statistics in the data for some observables in some energy-loss regions make it difficult to discuss trends. The fact that each data point represents a particular magnetic field setting also might contribute to small systematic errors from one setting to the next.

The design of the new medium energy resolution spectrometer (MRS) at LAMPF (Fig. 1) suggests that this instrument is ideal for nuclear response measurements in the continuum. We have initiated an experimental program using the MRS, whose purpose is to both commission and test the spectrometer over a wide energy range, and yield new physics through a measurement of a complete set of polarization transfer data for ${ }^{1} \mathrm{H},{ }^{2} \mathrm{H},{ }^{12} \mathrm{C}$, and ${ }^{40} \mathrm{Ca}$ at $20^{\circ}$ with energy losses extending to about $600 \mathrm{MeV}$. The entire energy loss region can be spanned using 
4 MRS magnetic field settings, and at $20^{\circ}$ the quasielastic peak is particularly well-defined.

The initial shakedown run for this experiment was done during Summer 1990, and considerably more than anticipated time was devoted to installing and commissioning the newly constructed MRS focal plane polarimeter (FPP) and understanding the optics of the beam line as well as those of the MRS itself. Although in principle the MRS-FPP is essentially the same as the HRS-FPP in theory of operation, in practice the MRS-FPP system is in many ways much more complex than that for the HRS-FPP system owing to the large momentum acceptance of the MRS (30\%) compared to the HRS (2\%). Thus, although it was possible to understand and operate the MRS system over a restricted region of the focal plane, the task of understanding it over the full momentum acceptance (30\%) was a time-consuming and difficult task. Off-line analysis of the data taken during this run provided a reasonable calibration and understanding of the MRS-FPP system, and it was anticipated that production data would be taken during Summer 1991. However, MRS safety concerns prohibited conducting the experiment during that time period. We expect that the experiment will be initiated once the shielding question is settled at the MRS.

\section{References}

[1] R. Fergerson et al., Phys. Rev. C 38, 2193 (1988).

\section{Figure Captions}

Figure 1: Schematic drawing of the medium resolution spectrometer (MRS) at LAMPF.

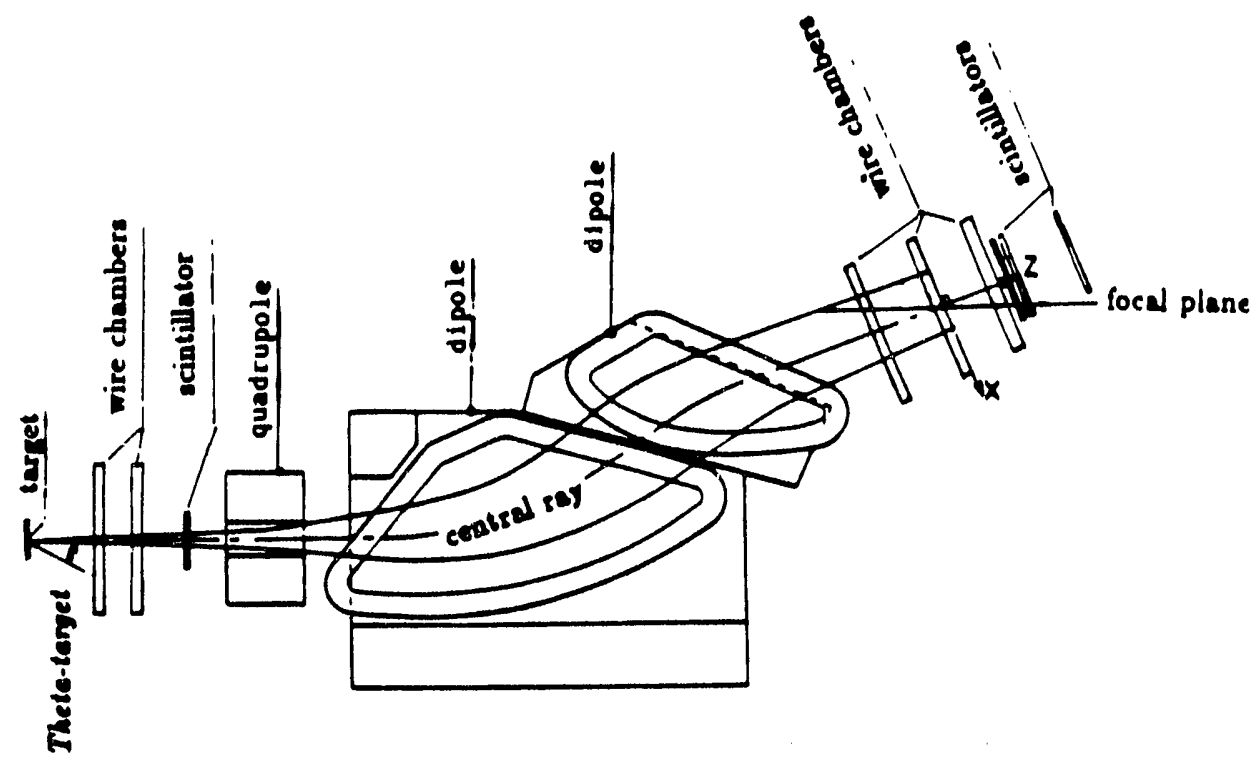




\title{
2.4 Polarized Nuclear Target Project
}

\author{
(G. Hoffmann, S. Worm, D. Read; LANL; ANL)
}

Summary: A copy of the PSI polarized nuclear target refrigeration system is being made at The University of Texas at Austin for use in medium energy hadronpolarized-nucleus scattering experiments at LAMPF and elsewhere. The construction phase of this project is nearing completion, and a working system is expected by mid-1992.

For some time, our group has had interest in exploring what new physics can be learned through use of polarized nuclear targets in medium energy scattering experiments. As an initial step in the realization of such experiments, we polarized ${ }^{13} \mathrm{C}$ and ${ }^{19} \mathrm{~F}$ using dynamic nuclear polarization technique: (DNP). ${ }^{1,2}$ The ${ }^{13} \mathrm{C}$ work lead to the development of the polarized target constructed at LAMPF ${ }^{3}$ and used for experiments 955,1023 , and 1025. This target was fabricated from one of the LAMPF setups originally designed to provide a polarized proton target for the LAMPF nucleon-nucleon program. Physically this setup (cryostat, plus supporting equipment such as vacuum pumps, dewars, NMR hardware, microwave hardware, etc.) was large, and considerable manpower and overhead was required in its use for $p+$ nucleus and $\pi+$ nucleus scattering experiments (several months setup/checkout time).

For the past 15 years the PSI group in Switzerland headed by Mango has been developing compact ${ }^{3} \mathrm{He}$ evaporation $/{ }^{3} \mathrm{He}-{ }^{4} \mathrm{He}$ dilution refrigerators for use with the polarized proton/deuteron experiments performed at PSI. Such compact setups would be ideal for the programs that seem to be starting up at LAMPF with polarized nuclear targets. Because of our longer term interest in performing medium energy experiments which utilize polarized nuclear targets, several years ago we decided to make a copy of the PSI refrigerator to replace the LAMPF designed system. Schematic diagrams of the cryostat, the ${ }^{3} \mathrm{He}$ evaporation unit, and the ${ }^{3} \mathrm{He}^{4} \mathrm{He}$ dilution refrigerator unit are shown in Figs. 1-3. PSI provided us with a complete set of engineering drawings for the system. They are also consulting with us concerning this technically difficult project. MP-7 at LAMPF and ANL are collaborating on this project.

The cryostat construction is complete, and approximately $85 \%$ of the ${ }^{3} \mathrm{He}$ evaporation unit has been made at this time. All components for the $70 \mathrm{GHz}$ microwave system required for DNP (at a magnetic field of $2.5 \mathrm{~T}$ ) have been acquired. We have also acquired most of the components required for the PIMR system. The microvax-CAMAC controlled NMR system developed for EXP955 will be updated and used for the new system. A $2.5 \mathrm{~T}$ superconducting split-coil magnet has recently been delivered by American Magnetics, Inc. The ${ }^{3} \mathrm{He}$ recirculation-storagesystem is currently in the assembly stage. The large pumps for the ${ }^{3} \mathrm{He}$ main 
pumping system have been purchased, and the pumping package (stands, valves, plumbing, etc., ) is presently being designed. Also, construction of the ${ }^{4} \mathrm{He}$ transfer system was recently completed.

The intention is that the setup will serve to test new materials for polarization feasibility studies as well as serve as the actual target for experiments at LAMPF and elsewhere. Its first use is anticipated to be for LAMPF EXP1206, "Polarized Proton Elastic Scattering from Polarized ${ }^{6} \mathrm{Li}$ at $500 \mathrm{MeV}$."

The target is designed to be service and user friendly. The sample holding device can be removed or inserted into the cryostat at $4 \mathrm{~K}$. PSI experience is that the target can be installed in the time period of one day. The comparable time period for the LAMPF setup is one month. The availability of the new PSI designed target, will greatly benefit future polarized nuclear target experiments at LAMPF. Shop work on this project competes with work on the EXP1079 project (see section 2.2) which presently has higher priority owing to the upcoming run anticipated this summer. We expect the EXP1079 work to be essentially complete by March 1992. Under this scenario, construction for the refrigerator project might be complete by Summer 1992, and commissioning would begin at that time. It is reasonable to expect that a working target would be available by late 1992 .

\section{References}

[1] J. J. Jarmer et al., Nucl. Inst. and Methds. A250, 576 (1986).

[2] D. Hill et al., Nucl. Inst. and Methds. A 277, 319 (1989).

[3] S. I. Penttilä et al., Eighth Int. Symp. of High-Energy Spin Physics, American Institute of Physics Conf. Proc. No. 187, New York, 1989, p. 1281.

[4] B. van den Brandt, J. A. Konter, and S. Mango, Nucl. Inst. and Methds. A289, 526 (1990).

\section{Figure Captions}

Figure 1: $\quad$ Schematic of PSI siandard cryostat with central tube for ${ }^{3} \mathrm{He}$ evaporation insert (Fig. 2) or dilution unit (Fig. 3).

Figure 2: $\quad$ Schematic of PSI ${ }^{3} \mathrm{He}$ evaporation unit and sample holding device.

Figure 3: $\quad$ Schematic of $\mathrm{PSI}{ }^{3} \mathrm{He}-{ }^{4} \mathrm{He}$ dilution refrigerator unit with corresponding eample holding device. 

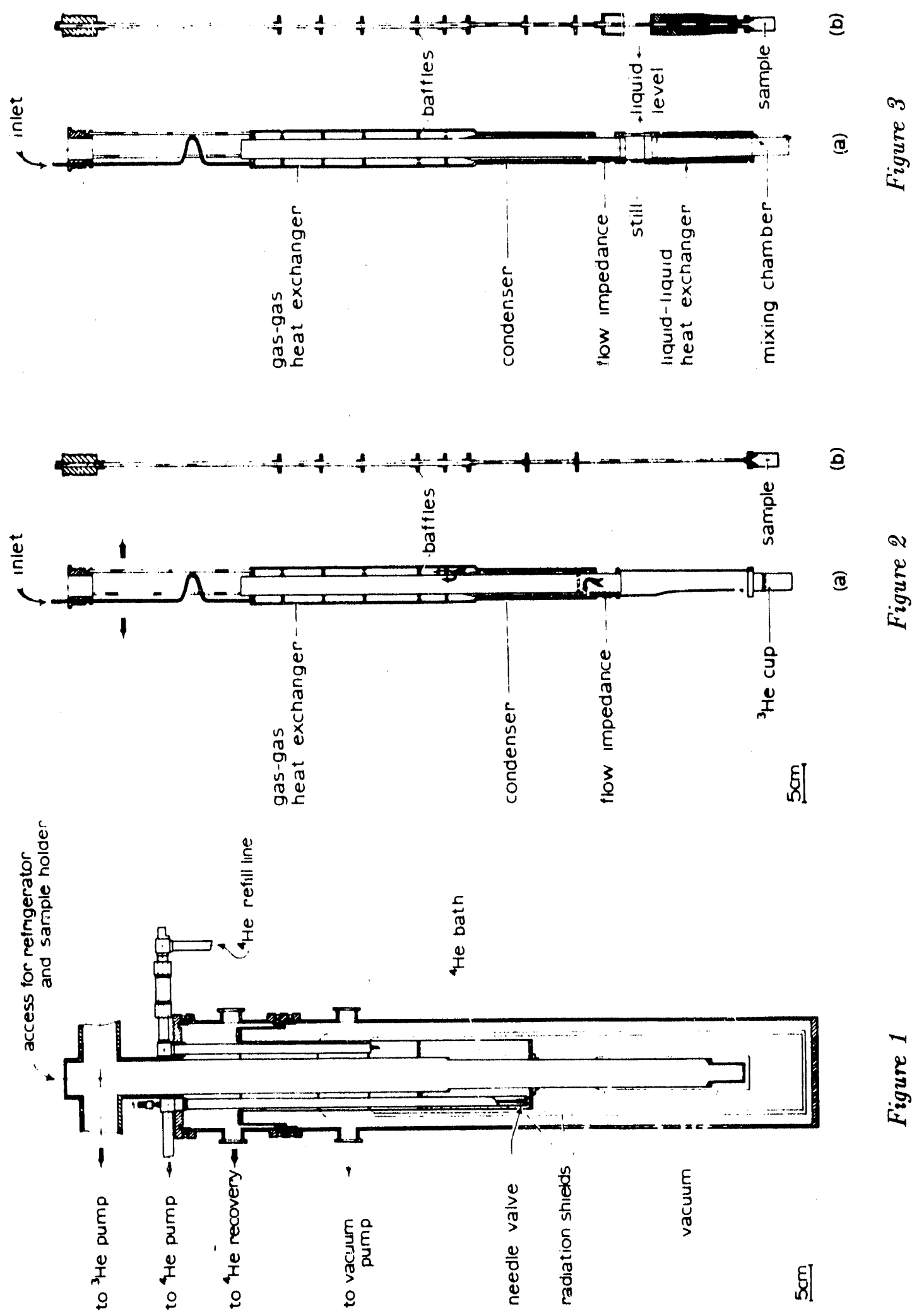


\subsection{BNL EXP791/871: Search For Very Rare $K_{L}$ Decays}

(G. W. Hoffmann, J. McDonough, S. Worm;

UCI; Stanford; Temple; Texas; William and Mary;

W. R. Molzon, J. L. Ritchie, S. G. Wojcicki, spokesmen)

Summary: In EXP871 a new search for the decays $K_{L} \rightarrow \mu e$ and $K_{L} \rightarrow e e$ will be made at the AGS. A single event sensitivity for $K_{L} \rightarrow \mu e$ below $10^{-12}$ will be reached and if no events are observed, an upper limit of about $2 \times 10^{-12}$ will be set. A few $K_{L} \rightarrow$ ee events should be observed, if this decay occurs at the rate predicted by the Standard Model, or more if new mechanisms contribute. About $10^{4} K_{L} \rightarrow \mu \mu$ decays will also be observed, thus improving considerably the accuracy of this branching ratio (BR). The principal improvements over the previous experiment (EXP791) are: increased beam (about a factor of 4) made possible by the Booster upgrade at the AGS, increased acceptance (more than a factor of 2), and improved efficiencies (more than a factor of 2). The most critical feature of the new experiment, the beam-plug, was extensively tested during the past year. The results from these tests confirmed the feasibility of the experiment and provided important data on the detector rates in the new experiment. We will construct an instrumented muon filter for the new experiment that will be used to limit trigger rates and to reduce backgrounds from $\pi-\mu$ misidentification.

\section{Physics Motivation}

Even though the Standard Model is extremely successful, it is considered to be incomplete. The reasons for this view can be traced to several problems: (1) there are nineteen free parameters in the theory, (2) there is no explanation of why quarks and leptons appear in three generations, or why there should not be more, (3) there is no explanation of how quarks and leptons are related, (4) there is a technical difficulty known as the "gauge hierarchy problem," which refers to the need for unnatural fine-tuning of parameters, and (5) there is no unification of the strong and electroweak interactions (and gravity is not in the picture at all). A number of proposed extensions of the Standard Model have been suggested including supersymmetry, ${ }^{1}$ technicolor, ${ }^{2}$ right,-left symmetry, ${ }^{3}$ horizontal gauge symmetry, ${ }^{\mathbf{4}, 5}$ compositeness, ${ }^{6}$ and some superstring inspired models. ${ }^{7}$ Typically, conservation of lepton number is violated in these extensions.

It is appropriate to consider alternative searches for lepton number violation and related physics. These include: (1) $\mu \rightarrow e \gamma$, (2) $\mu \rightarrow e e e,(3) \mu A \rightarrow e A$ (muon conversion on a nucleus), and (4) $\Delta m_{K}$ (the $K_{L}-K_{S}$ mass difference). Processes such as $\mu \rightarrow e \gamma$ and $\mu \rightarrow e e e$ only involve leptons (no quarks) in the initial or final state, so that any interaction depending upon the participation of quarks (e.g., generation number conserving interactions, composite models) are not probed at all. The process $\mu A \rightarrow e A$ also does not involve a generation-change in the quark 
sector. The small value of $\Delta m_{K}\left(3.5 \times 10^{-15} \mathrm{GeV}\right)$ provides a serious constraint in a broad range of models. However, a sensitivity of $10^{-12}$ for $K_{L} \rightarrow \mu e$ is beginning to be competitive with $\Delta m_{K}$. Also, leptoquark exchange is not constrained at all by $\Delta m_{K}$.

To quantify this discussion we use the model of Cahn and Harari ${ }^{5}$ for generationchanging gauge hosons to illustrate the mass scales probed by each of these processes. Table I lists the best present limit for each process and the mass scale probed. The limit given for $K_{L} \rightarrow \mu e$ is the lowest limit yet reported from EXP791 (Ref. 8), but is not the final EXP791 result from the full (three years) data set. The bottom row shows the mass reach of the new experiment. The mixing angles $(\beta)$ characterize the generation mixing in the model and are unconstrained. If generation number is approximately conserved, then the mixing angles would be small. The mixing angles do not appear in the expressions for the mass reach for the two kaon decays. It is easy to conclude from these considerations that a search for $K_{L} \rightarrow \mu e$ approaching the $10^{-12}$ level is important and that the prospects for observing $K_{L} \rightarrow \mu e$ in this new search are not ruled out by previous limits.

\begin{tabular}{|c|c|}
\hline Process and Present Value & Mass Reach \\
\hline$\Delta m_{K}\left(3.5 \times 10^{-15} \mathrm{GeV}\right)$ & $400 \mathrm{TeV} \sin \beta_{D}$ \\
\hline$B r(\mu \rightarrow e \gamma)<1.7 \times 10^{-11}$ & $20 \mathrm{TeV}\left|\sin \beta_{L} \cos \beta_{L}\right|^{1 / 2}$ \\
\hline$B r(\mu \rightarrow e e e)<1 \times 10^{-12}$ & $80 \mathrm{TeV}\left|\sin \beta_{L} \cos \beta_{L}\right|^{1 / 2}$ \\
\hline$\Gamma(\mu \cdot A \rightarrow e A) / \Gamma(\mu A \rightarrow \nu A)<4.6 \times 10^{-12}$ & $190 \mathrm{TeV}\left|\sin { }^{\circ} L U-\sin \beta_{L D}\right|^{1 / 2}$ \\
\hline$B r\left(K^{+} \rightarrow \pi^{+} e^{-} \mu^{+}\right)<2.1 \times 10^{-10}$ & $40 \mathrm{TeV}$ \\
\hline$B r\left(K_{L} \rightarrow \mu e\right)<8.4 \times 10^{-11}$ & $70 \mathrm{TeV}$ \\
\hline \hline$B r\left(K_{L} \rightarrow \mu e\right)<2.0 \times 10^{-12}$ (this proposal $)$ & $180 \mathrm{TeV}$ \\
\hline
\end{tabular}

Table I: Mass reach of separate lepton number violating processes in the Cahn and Harari model. The $\beta$ 's are generation mixing angles and are unconstrained.

Finally, while the theoretical considerations are encouraging, the ultimate justification for an improved $K_{L} \rightarrow \mu e$ search is simply to test the rule of separate lepton number conservation to the highest precision possible with existing accelerators and detectors.

The allowed, but very rare decay, $K_{L} \rightarrow e e$, is expected ${ }^{9}$ to occur at a branching ratio of about $3 \times 10^{-12}$; the present limit from EXP791 is $<1.1 \times 10^{-10}$. The range between these values is a window for observing a possible new flavor changing neutral current interaction.

\section{Results of EXP791}

The final data set, taken in 1990 , is in the final stages of analysis. Papers are in preparation to report not only the 1990 results but also the combined 1988-1990 results. Preliminary results will be given here. 
The EXP791 detector layout is shown in Fig. 1. The $K_{L}$ beam is produced from $24 \mathrm{GeV}$ protons striking a $\mathrm{Cu}$ target. Photons are converted in $\mathrm{Pb}$ foils and charged particles are swept away by a series of magnets. The neutral particles that remain $\left(95 \%\right.$ neutrons, only $5 \% K_{L}$ ) are collimated into a $5 \mathrm{mrad} \times 20 \mathrm{mrad}$ beam with a central angle that is 2.75 degrees above the plane of the proton beam. Approximately $3 \%$ of the $K_{L}$ 's decay in the vacuum decay region. The decay products are tracked, using five sets of low-mass drift chambers, through a doublearm, two magnet spectrometer. The magnetic fields are set to provide opposite $300 \mathrm{MeV} / \mathrm{c}$ momentum kicks. Downstream of the last drift chamber are a pair of scintillation counters that are used in the trigger. Between the pair of trigger counters is a gas Cerenkov that is used to identify electrons. Also used for electron identification is a $\mathrm{Pb}$ glass array. Muons are identified by a scintillation counter behind $91 \mathrm{~cm}$ of $\mathrm{Fe}$ and by use of the muon rangefinder which consists of 13 planes of gas counters placed within a 13 meter marble range-stack.

The lowest-level trigger is formed by a coincidence requiring signals, on both the left and right side, from both the upstream and downstream trigger scintillation counters. An overlap of these coincidences with signals from the upstream drift chambers is made to ensure that the tracks originate in the decay region rather than from $K_{L}$ decays that occur within the spectrometer. The next trigger level requires signals from the lepton identification counters; the Cerenkov counter and the muon scintillator hodoscope. These events are then processed on-line by eight $3081 \mathrm{E}$ processors where tracks are reconstructed. Events that satisfy invariant mass requirements and have a reconstructed momentum vector that is consistent with a $K_{L}$ decay are written to tape for off-line processing.

The best published limits ${ }^{10}$ on $K_{L} \rightarrow \mu e$ and $K_{L} \rightarrow e e$ branching ratio measurement at the $90 \%$ confidence level are $9.4 \times 10^{-11}$ and $1.6 \times 10^{-10}$, respectively. Analysis of the 1989 data is complete and will be published with the 1990 data. No $K_{L} \rightarrow \mu e$ or $K_{L} \rightarrow e e$ candidates were found. The preliminary results are:

\begin{tabular}{|l|c|c|}
\hline Year & $K_{L} \rightarrow \mu e$ & $K_{L} \rightarrow e e$ \\
\hline 1990 & $7.1 \times 10^{-11}$ & $8.0 \times 10^{-11}$ \\
\hline $1988-1990$ combined & $3.3 \times 10^{-11}$ & $4.1 \times 10^{-11}$ \\
\hline
\end{tabular}

Table II: $90 \%$ confidence level limits for $K_{L}$ branching ratios from 1990 data and the combined 1988-1990 data.

The most precise published ${ }^{11}$ branching ratio measurement of $K_{L} \rightarrow \mu \mu$ is from the 1989 data set. The 1989 result was:

$$
B R\left(K_{L} \rightarrow \mu \mu\right)=(7.6 \pm 0.5 \text { (stat.) } \pm 0.4 \text { (syst.) }) \times 10^{-9} .
$$

The preliminary 1990 result is:

$$
\left.B R\left(K_{L} \rightarrow \mu \mu\right)=(6.96 \pm 0.40 \text { (stat. }) \pm 0.22(\text { syst. })\right) \times 10^{-9} .
$$


Three experiments have been active over the last few years in the search for these rare modes: BNL EXP780, KEK EXP137, and BNL EXP791. In general, the sensitivity to $K_{L} \rightarrow \mu e$ and $K_{L} \rightarrow e e$ events in EXP791 is about an order of magnitude better than that of BNL EXP780 and over a factor of two better than the KEK experiment. The number of $K_{L} \rightarrow \mu \mu$ events are about 8 and 200 in BNL EXP780 and KEK EXP137, respectively, in comparison with about 700 in EXP791 (1988 - 1990 three year tolal).

\section{Overview of the New Experiment}

The Program Advisory Committee at BNL has approved our request for an engineering run in 1993 followed by two years of physics data. The experiment will improve upon EXP791 by more than an order of magnitude in sensitivity and set a limit on $K_{L} \rightarrow \mu e$ below $2 \times 10^{-12}$ if no signal is observed. At this sensitivity, we should observe several $K_{L} \rightarrow e e$ events. The key features of the new experiment are:

- The decay volume will be lengthened.

- The tracking detectors will be modified to be efficient at high rates.

- Detector apertures will be increased by using a new magnet configuration. The two dipoles will be operated with opposite fields, but tuned to provide a net $p_{T}$-kick of about $220 \mathrm{MeV}$, which will make daughters from 2-body $K_{L}$ decays parallel to the beam direction. (This parallelism will be used in forming the trigger.) The events that will be accepted are the so-called "in-bends", in which both charged particles bend toward the beam in the upstream dipole. The "out-bends", which first bend away from the beam, will not be accepted in this magnet configuration.

- A beam stop, or plug, will be inserted in the upstream dipole magnet (96D40) to stop the neutral beam.

- A new Čerenkov counter and an instrumented iron muon filter will be built.

- The muon rangefinder will be reconfigured and reused.

- The triggers will be improved.

- The acceptance will be increased.

The layout of the new experiment is shown in Fig. 2. In the following several of these improvements are discussed.

Precise tracking is essential in this experiment since background rejection relies on precise kinematic reconstruction. The EXP871 drift chambers will present difficulties largely in the module immediately upstream of the plug in the new geometry. Neglecting any increased rate from neutrons or photons originating in 
the plug, the instantaneous intensity will increase by a factor between 4 and 6 due to the increased beam flux and longer vacuum decay volume. The chambers ran at rates in excess of $10 \mathrm{MHz}$ in EXP791 and drew about $50 \mu$ amps in current. The two detectors upstream of the beam-plug will consist of $0.5 \mathrm{~cm}$ straw tube drift chambers. We may place the most-upstream of these detectors in vacuum to obtain the best possible vertex resolution but no one has done this before so there are technical challenges that must be overcome. Gas with a high drift velocity will be used in all drift chambers. The combination of smaller cell size and faster gas will permit the tracking detectors to operate efficiently with eight times higher rate as in the EXP791 drift chambers.

The use of two sequential magnets in a $K_{L} \rightarrow \mu e$ search is critical, since the decay in flight of a $\pi$ (from $K_{e 3}$ ) inside the magnet of a single magnet spectrometer could simulate false $K_{L} \rightarrow \mu e$ events. The second magnet permits an independent momentum measurement, which is very powerful in rejecting such events. In the new geometry, the sequential magnets will be tuned to provide a net $p_{T}$-kick of $220 \mathrm{MeV}$, which will cause accepted $K_{L} \rightarrow \mu e$ events to have both charged tracks parallel to the beam direction downstream of the second dipole. However, rather than have two relatively weak fields providing a total $p_{T}$-kick of $220 \mathrm{MeV}$, the first dipole will "over-bend" $\left(\Delta p_{T}=440 \mathrm{MeV}\right)$ and be corrected in the second dipole $\left(\Delta p_{T}=-220 \mathrm{MeV}\right)$, so that tracks will traverse a much larger total $\int \mathbf{B} \cdot \mathbf{d} \ell$. This permits a much better determination of track momentum. The optics of the system of dipole magnets will enhance the acceptance of the spectrometer. We plan to reuse the larger of the two dipole magnets used in EXP791 (96D40) as the upstream dipole in the new experiment. A second large dipole magnet will be needed. We believe that a magnet at FermiLab satisfying our needs will be available so it should not be necessary to build a new magnet.

The most novel feature of the new experiment involves placing a beam dump, or plug, inside the spectrometer to stop the neutral beam and the bulk of secondary particles that are created within it. This will reduce the rates due to $K_{L}$ decays and neutron interactions which would otherwise occur in the detectors far downstream of the beam plug position. The advantages of this new approach are: (1) the acceptance is improved a factor of 2.1 because there is no longer a need for the downstream detectors to have an opening between them through which the neutral beam passes, (2) the beam plug will reduce the rates in the downstream detectors, (3) particle identification should be cleaner, owing to the much lower rates in the lead glass and muon detectors, (4) the parallelism of tracks from 2-body decays will provide a large suppression in the Level 1 trigger rates (allowing more time per event for the online processor) and make it easier to group signals from trigger counters, the Čerenkov counter, and the muon system, (5) the radiation dose on the lead glass will be reduced by more than an order of magnitude, and (6) making detectors continuous across the beam centerline will eliminate the degradation of performance that typically occurs near detector edges (e.g., side leakage of showers in the lead glass, multiple scattering of muons into the beam region in the muon 
steel or rangefinder). A great deal of effort has gone into designing and testing the beam-plug. This will be discussed in the next section.

Good particle identification is critical in a $K_{L} \rightarrow \mu e$ search, because the major backgrounds involve misidentifying either one or both of the charged daughters in the copious $K_{L} \rightarrow \pi e \nu$ decay. In EXP791, electron identification was accomplished with a threshold Cerenkov counter and a lead glass array, where muon identification was done with a scintillator hodoscope behind an iron wall and a muon rangefinder (in which muons below about $6 \mathrm{GeV}$ were stopped, allowing a comparison between measured upstream momentum and range). In the new experiment, each of these devices will be upgraded, rebuilt, or reconfigured to improve performance.

The Cerenkov counter will be of the atmospheric-pressure, gas threshold type, to separate electrons from pions and muons. It will cover an area of about 4 square meters, centered on the beam line, and have low mass, so that multiple scattering is small. To reduce accidental coincidences, signals from the fast photomultiplier tubes will be used in the trigger which will require the $\mathrm{x}$-positions of the hits in the Čerenkov counter and trigger counters be consistent.

The new Cerenkov counter is expected to have higher efficiency than the EXP791 counter (98\% versus $89-90 \%)$ due to careful attention to optical design and execution, mirror quality, light funnel shape and reflectivity, selection of phototubes and wavelength shifter. It will also have more segmentation (32 versus 16 cells) and lower knock-on probability (mainly due to less mass in the upstream detectors). The beam-plug test showed that special attention must be paid to shielding the tubes from the ambient flux of neutrons and photons to avoid excess rates in the Čerenkov counter.

The lowest momentum at which muons were accepted in EXP791 was 1.5 $\mathrm{GeV} / \mathrm{c}$. In the new experiment, we plan to instrument the meter of steel which follows the lead glass array with layers of scintillation counters. This will permit a lower $(1 \mathrm{GeV} / \mathrm{c})$ muon momentum threshold which will increase the acceptance by about $10 \%$. The instrumented muon filter will also improve our ability to trigger on muons and provide good timing information on muon tracks that project into the muon rangefinder (which provides effectively no time information because drift times are long and the hits are latched). The transverse coverage of this device will be roughly $2 \mathrm{~m}$ by $2 \mathrm{~m}$, centered on the beam in both $x$ and $y$. Our group will bear the main responsibility for this device. The details of the design are given in a later section.

The muon rangefinder ${ }^{12}$ in EXP791 consisted of two separate detectors on either side of the beam. They each consisted of marble (and some aluminum) slabs ( $2.25 \mathrm{~m}$ wide and $3 \mathrm{~m}$ vertically) with extruded aluminum drift tube chambers interspersed at intervals that provided a constant $\Delta p / p$ resolution of $10 \%$ for stopping muons. There were 13 pairs of $x$ and $y$ planes of these chambers on each side of the beam. Comparing the measured muon range to that expected from the momentum measured in the magnetic spectrometer upstream provides rejection against pion decay downstream of the magnets. 
In the new geometry, the muons from accepted $K_{L} \rightarrow \mu e$ and $K_{L} \rightarrow \mu \mu$ decays will enter the muon identification detectors parallel to the beam direction so that the transverse extent of the muon coverage need not be as large as in EXP791 so that only one side of the muon rangefinder is required. This will free 13 detector planes which can be used to increase the segmentation, so that roughly $5 \%$ resolution in $\Delta p / p$ can be obtained. We will also use some of these chambers to extend the range of momentum covered to $\geq 8 \mathrm{GeV}$.

One of the clearest strengths of this experiment over EXP791 is the improvement in the triggering. Briefly, the important features are: (1) The parallelism condition will provide a large suppression of semi-leptonic decays and will be relatively insensitive to accidentals and noise triggers. (2) Due to the parallel trajectories of triggering tracks, it will be easy to impose tight constraints on associated particle identification (Čerenkov and muon filter) signals. (3) Rates in the downstream trigger planes and particle identification detectors from genuine charged tracks will be considerably suppressed by the plug. In addition, singles rates will be lower in the muon counters even though the beam intensity increases by a factor of four.

The acceptance of the new detector has been calculated using Monte Carlo. Overall a factor of two improvement over EXP791 is expected. This arises from a number of sources including the larger decay volume, the new magnets and parallel-to-beam trajectories for the $K_{L} \rightarrow \mu e$ tracks, and the use of the central regions of the detectors (with beam plug). We have investigated the impact of a wider beam plug on the overall acceptance and find that only a $5 \%$ change in acceptance results from a $13 \%$ change in plug width.

\section{Sensitivities and Backgrounds}

The sensitivities for the new experiment are scaled from a well-understood (1989) data set obtained in EXP791. The new experiment depends on increased acceptance, running at higher beam intensity, and on a variety of improvements to individual systems which will improve efficiencies, reduce deadtimes, etc. The separate factors are listed in Table III.

The decays $K_{L} \rightarrow \mu e, K_{L} \rightarrow e e$, and $K_{L} \rightarrow \mu \mu$ have different acceptances and are affected differently by changes in particle identification efficiencies. The relative improvements in the sensitivities for each decay channel are listed in Table IV, these results being derived from Table III. The expected sensitivities for the new experiment are also shown.

In a high sensitivity experiment background rejection is of paramount concern. For example, the copious $K_{L} \rightarrow \pi e \nu$ followed by $\pi \rightarrow \mu \nu$, provides an abundant source of $K_{L} \rightarrow \mu e$-like events. These are rejected by requiring no missing transverse momentum, eliminating trajectories with kinks (i.e., $\pi$ decay), and determination of invariant mass which is a maximum of $489 \mathrm{MeV}$ for the false event mentioned here. Another background source is misidentification of the $\pi$ as an $e$ and the $e$ as a $\mu$ in the $K_{L} \rightarrow \pi e \nu$ decay. Therefore precise kinematic event 


\begin{tabular}{|l|c|c|c|}
\hline & EXP791 1989 Data & New Experiment & Increase \\
\hline$K_{L} \rightarrow \mu . e$ acceptance & 0.0113 & 0.0242 & 2.14 \\
\hline$K_{L} \rightarrow e e$ acceptance & 0.00873 & 0.0224 & 2.57 \\
\hline$K_{L} \rightarrow \mu \mu$ acceptance & 0.0149 & 0.0283 & 1.90 \\
\hline Protons on target & $3.8 \mathrm{Tp}$ & $15 \mathrm{Tp}$ & 3.95 \\
\hline L1 trigger efficiency & 0.72 & 0.95 & 1.32 \\
\hline L3 trigger efficiency & 0.64 & 0.95 & 1.48 \\
\hline Ćrenkov eíectron efficiency & 0.89 & 0.96 & 1.08 \\
\hline PBG electron efficiency & 0.95 & 0.97 & 1.02 \\
\hline Running efficiency & 0.875 & 0.92 & 1.05 \\
\hline Running time & 17 weeks & 38 weeks (2 years) & 2.24 \\
\hline
\end{tabular}

Table III: Improvement factors of the new experiment over the 1989 EXP791 run.

\begin{tabular}{|l|c|c|c|}
\hline & EXP791 1989 Data & Improvement & Proposal \\
\hline$K_{L} \rightarrow \mu e$ sensitivity (single event) & $3.6 \times 10^{-11}$ & 43 & $8.4 \times 10^{-13}$ \\
\hline$K_{L} \rightarrow e e$ sensitivity (single event) & $4.8 \times 10^{-11}$ & 56 & $8.5 \times 10^{-13}$ \\
\hline$K_{L} \rightarrow \mu \mu$ observed & 284 events & 34.5 & 9800 events \\
\hline
\end{tabular}

Table IV: Sensitivity of the new experiment.

reconstruction and particle identification is essential. Monte Carlo simulations for the new detector with tracking in the decay volume, redesigned drift chambers and new magnets indicate that some improvement in resolution will result. In addition to improved resolution, other reasons why background rejection in the new experiment should be better than in EXP791 include the following: (1) The larger magnetic field in the upstream dipole will minimize the effect from pion decay in the downstream dipole. (2) The drift chamber located downstream of the Čerenkov counter will provide additional rejection of pions which decay downstream of the magnets. (3) Finer rangefinder segmentation $(\Delta p / p \simeq 5 \%)$ will provide better discrimination against downstream pion decays. (4) Better discrimination of pions against electrons should be possible with both the Cerenkov counter, which will have a lower knock-on probability and higher segmentation, and the lead glass, which will have improved resolution. (5) The lower rates in the muon systems with the beam plug will considerably reduce the probability of mistaking an ele:tron for a muon. We are therefore reasonably optimistic that backgrounds can be suppressed to necessary levels. 


\section{The EXP871 Beam Plug}

The critical feature of the new experiment is the beam-plug that will be placed inside the first spectrometer magnet. It must stop the neutral beam; photons, neutrons and undecayed $K_{L}$ 's, which will reduce the rates in the downstream particle identification detectors. Since drift chambers will be placed very close to the beam-plug, the plug must prevent the secondary particles, produced from the high-energy neutral beam particles, from escaping and causing excess rate in the chambers. The plug must accomplish all this with a minimal transverse extent; any increase in the plug width beyond $40 \mathrm{~cm}$ causes a loss of acceptance.

The design and testing of the beam-plug was the major effort for our group during the last year. Optimization of the design was performed using a simulation program, called CALOR89, from Oak Ridge National Laboratory. The beamplug was extensively tested at Brookhaven during the 1991 AGS proton cycle. In addition to rate measurements using the EXP791 detectors, we built several special detectors to measure the number and energy-spectrum of the neutrons that were produced by the plug. These detectors; Bonner spheres, liquid scintillators, and ${ }^{3} \mathrm{He}$ counters were used both to identify the source of drift chamber rates and also as a test of the Monte Carlo program. Our group was responsible for these special detectors and the acquisition and analysis of the data provided by them. The beam-plug design and test are described below.

\section{Beam-plug Design}

The ideal material for the central part of the beam-plug is tungsten. It has an extremely short hadronic interaction length, $9.6 \mathrm{~cm}$, which is surpassed only by very expensive materials such as platinum. In addition to its short hadronic interaction length, tungsten has two other very valuable properties. First, it has a large inelastic cross-section for neutrons down to energies of $1 \mathrm{MeV}$. Since, for each incident high-energy hadron, hundreds of "boil-off" neutrons are produced with energies less than $10 \mathrm{MeV}$ it is important to attenuate them before they interact in the drift chambers via $(n, p)$ scattering in the Ar-ethane gas. A second useful property of tungsten is its short electromagnetic interaction length. Although, with a $\mathrm{Z}=74$, it is not as effective per electron as $\mathrm{Pb}(\mathrm{Z}=82)$; the higher density of tungsten causes it to have a shorter attenuation length than $\mathrm{Pb}$.

For neutrons with energy below $1 \mathrm{MeV}$, elastic scattering from hydrogen is the most effective method for moderating the neutron energy. Materials with a high density of hydrogen, such as polyethylene or water, are usually doped with small amounts of boron or lithium because of the high absorption cross-section of these elements for thermal neutrons. One disadvantage of boron, however, is that a 477 $\mathrm{keV}$ photon is usually produced in the neutron capture process. These photons can be attenuated by surrounding the neutron-capture material with $\mathrm{Pb}$.

The beam-plug can be crudely designed using these materials; a core of tungsten surrounded by a hydrogen-dense material, doped with an element with a large 
capture cross-section for thermal neutrons, which is itself covered with a layer of $\mathrm{Pb}$ to attenuate photons. The exact design is much more difficult because the transverse width that can be used is limited to less than $40 \mathrm{~cm}$. Therefore, we must balance the amounts of each material so that the induced drift chamber rates are minimized. The specific questions that must be answered are:

- How much tungsten should be used? Tungsten very efficiently attenuates charged particles, photons, and neutrons down to $1 \mathrm{MeV}$; but a beam-plug of pure tungsten would emit an enormous number of low-energy neutrons which have large cross-sections for scattering in the drift chamber gas.

- What type of hydrogen-dense material should be used? Pure polyethylene has the highest hydrogen density commercially available and would be the most effective to moderate neutrons but would allow many thermal neutrons to escape. Doping the polyethylene will prevent the escape of the thermal neutrons but at the expense of lower hydrogen density.

- Should the doping element be boron or lithium? Boron-doped polyethylene has a higher hydrogen density than lithium-doped polyethylene but also produces a photon which is not the case for lithium.

- How much $\mathrm{Pb}$ should be used? $\mathrm{As}$ more $\mathrm{Pb}$ is added the photon energy spectrum becomes harder so every additional layer of $\mathrm{Pb}$ will be much less effective than the one before it.

- Where along the beam direction should the beam-plug begin? Moving the beam-plug downstream will increase the drift chamber rates there but will decrease the upstream drift chamber rates.

These specific questions can only be answered using a simulation program. The best one for our purposes is CALOR89, which consists of four different parts. The hadronic interactions are simulated with FLUKA at high energy and by HETC at lower energy $(<5 \mathrm{GeV})$. Photons and low-energy neutrons produced in these interactions are stored. The neutrons are then transported, using MORSE, down to thermal energies. Photons that are produced in this code are added to the photons from the hadronic interactions and are transported using EGS4. The probabilities for the particles that escape the beam-plug to interact in the drift chambers is then calculated which permits us to quantify the effects of varying beam-plug parameters.

The ratio of neutrons: $K_{L}$ in the neutral beam for a production angle of 2.75 degrees is approximately 20:1. In addition, the neutrons produce more secondary particles since they tend to be more energetic than the $K_{L}$ 's. The input to the Monte Carlo simulations therefore includes only the neutrons. The incident neutron energy spectrum was generated using a GEANT simulations and had an average energy of $12 \mathrm{GeV}$. 
The design of the optimized beam-plug is shown in Fig. 3. It was installed at BNL during a 10 week test run from April-June, 1991.

\section{Beam Plug Test Results}

The major goal of the test was to measure the rates in nearby drift chambers for a well-designed beam-plug. With this information we are able to determine that EXP871 is feasible and we use the data obtained to design the tracking detectors. If, in addition to the drift chamber rates, we acquire data that identifies the particles that escape from the beam-plug then we can use that information to improve the beam-plug. The special detectors that we assembled at Texas provided us with that additional information.

Most of the detectors that were used were intended to measure slow neutrons. The Bonner spheres, several sizes of polyethylene spheres that moderate neutrons with energy-dependent efficiencies, provided a measure of the fluence and energy spectrum of neutrons between thermal and $26 \mathrm{MeV}$. The ${ }^{3} \mathrm{He}$ counters, which detect the decay products from the reaction ${ }^{3} \mathrm{He}(\mathrm{n}, \alpha)^{3} \mathrm{H}$, were used to test the low-energy Bonner sphere results. The liquid scintillators tested the higher-energy Bonner sphere results since they were able to detect neutrons above $1 \mathrm{MeV}$ through $(\mathrm{n}, \mathrm{p}$ ) elastic scattering and identify them because the slow protons ionize much more than minimum ionizing particles causing different pulse shapes for these events. Typically the different neutron flux measurements agreed to better than $50 \%$ so we believed the Bonner sphere results to that level.

The liquid scintillators also provided the photon and charged particle measurements. Charged particles were identified using thin plastic scintillators in coincidence with the liquid scintillator. Photons were defined to be those particles with no plastic scintillator in coincidence and with a pulse-shape consistent with a minimum ionizing particle.

Once the energy spectrum of the neutrons and photons was known, the induced rates on the drift chambers could be calculated by multiplying the number in a given energy bin times the interaction probability for a neutron o: photon of that, energy. For the charged particles only the total number was necessary since the probability for a charged particle to interact in the drift chamber was unity.

Table V contains, for two different beam-plug configurations, the actual drift chamber rates, the calculated rates from the Bonner spheres and liquid scintillators, and the predicted rates from the CALOR89 Monte Carlo simulations. The simulation results agree well with the results from the neutron, photon, and charged particle measurements. However, both of these results seem to explain only half of the total drift chamber rates. Part of the cause of this discrepancy is due to the low efficiency for the charged particle definition but the possibility that we have overlooked some interaction process is still being investigated. The simulations and our measurements indicate that charged particles are the major cause of drift chamber rates.

The real measure of the feasibility of the beam-plug is the rates in the EXP871 


\begin{tabular}{crr}
\hline Upstream position & & \\
& metal plug & shielded plug \\
\hline Actual measured rate & $\mathbf{3 9 4 2}$ & $\mathbf{1 3 9 9}$ \\
\hline Calculated from special & & \\
detectors (total) & $\mathbf{1 1 5 1}$ & $\mathbf{6 3 4}$ \\
(from neutrons) & 260 & 55 \\
(from photons) & 146 & 76 \\
(from charged) & 745 & 503 \\
\hline Estimated from & & \\
Monte Carlo (total) & $\mathbf{1 4 5 5 - 2 9 1 0}$ & $\mathbf{3 2 0 - 6 4 0}$ \\
(from neutrons) & $175-350$ & $25-50$ \\
(from photons) & $30-60$ & $45-90$ \\
(from charged) & $1250-2500$ & $250-500$ \\
\hline
\end{tabular}

Downstream position

\begin{tabular}{crr} 
& metal plug & shielded plug \\
\hline Actual measured rate & $\mathbf{2 1 5 0}$ & $\mathbf{8 6 5}$ \\
\hline Calculated from special & & \\
detectors (total) & $\mathbf{5 9 7}$ & $\mathbf{4 5 3}$ \\
(from neutrons) & 118 & 17 \\
(from photons) & 79 & 38 \\
(from charged) & 597 & 398 \\
\hline Estimated from & & \\
Monte Carlo (total) & $\mathbf{1 8 0 - 3 6 0}$ & $\mathbf{1 3 5 - 2 7 0}$ \\
(from neutrons) & $50-100$ & $15-30$ \\
(from photons) & $5-10$ & $20-40$ \\
(from charged) & $125-250$ & $100-200$ \\
\hline
\end{tabular}

Table V: The actual rates for drift chambers near the plug for two different plug configurations; tungsten without polyethylene and tungsten with polyethylene and $\mathrm{Pb}$. The rates due to neutrons, photons and charged particles are given (a) calculated from data taken with Bonner spheres and liquid scintillators and (b) estimated from the results of the Monte Carlo simulations. The units are (drift chamber clusters)/B5T1 where B5T1 is a beam counter telescope. The normalization to the Monte Carlo input is not well known so a range is given. 
detectors. These detectors do not exist yet so the rates in the EXP791 detectors were measured during the test. As can be seen in Table VI, the particle identification counters generally had much lower rates after the beam-plug was installed. The only exception was the Cerenkov counter which had a marginal increase in rate. The rates doubled for drift chambers near the beam-plug which, when the beam intensity increases by a factor of four, means that the new tracking chambers will have to handle eight times the rate are the EXP791 drift chambers. This will be accomplished by using straw detectors with diameters half as large as the cell size in the EXP871 detectors and by using gas with a faster drift velocity than Ar-ethane.

\begin{tabular}{|l|c|}
\hline Detector & Ratio (with plug/without plug) \\
\hline Drift Chamber 2 & 1.5 \\
\hline Drift Chamber 3 & 1.9 \\
\hline Drift Chamber 4 & 2.2 \\
\hline Drift Chamber 5 & 1.2 \\
\hline Trigger Counter 1 & 1.6 \\
\hline Cerenkov counter & 1.1 \\
\hline Trigger Counter 2 & 0.46 \\
\hline Lead-glass Array & 0.20 \\
\hline Muon Hodoscope & 0.15 \\
\hline Muon Rangefinder & 0.10 \\
\hline
\end{tabular}

Table VI: The effect of the beam-plug on the rates in the EXP791 detectors displayed as the ratio of rates (with/without) the beam-plug.

\section{Instrumented Muon Filter}

The primary functions of the instrumented muon filter (IMF), are (1) to provide a signal to the Level 1 . logic which indicates the passage of a parallel muon with momentum greater than $1 \mathrm{GeV} / \mathrm{c}$, and (2) to allow determination of muon momentum in the $1 \mathrm{GeV} / \mathrm{c}$ to $1.5 \mathrm{GeV} / \mathrm{c}$ range to about $5 \%$ via range measurements in the iron. The detector will consist of about 2 meters of iron plates, three planes of $x-y$ scintillators, and 19 MRG panels.

A fundamental limitation in the transverse dimensions of the muon identification system exists since components of the MRG of EXP791 will be reused in EXP871. Because of this it is important that effects of multiple Coulomb scattering in the instrumented iron filter be well understood. The detector design should minimize the number of muons that escape from the rangefinder before reaching a point that provides a valid range-measurement. Adjustable parameters include the amount and type of material that is used upstream of the rangefinder. We have studied multiple scattering effects both analytically and with Monte Carlo 
simulations and conclude that air gaps must be minimized, and material such as iron should be used rather than using the existing marble immediately after the scintillator plane at the $1 \mathrm{GeV} / \mathrm{c}$ position.

Multiple scattering also limits the usefulness of a parallelism muon trigger in Level 1, mostly because of the scattering that takes place in the lead-glass array $(\mathrm{PbG})$. The multiple scattering in the $\mathrm{PbG}$ has an enhanced effect because a distance of approximately one meter is required between it and the iron for photomultiplier tubes, bases, and cables. The transverse RMS spread at both the stopping point and the $1 \mathrm{GeV} / \mathrm{c}$ trigger point, for an incident monoenergetic, pencil beam of muons (1000 per momentum bin), has been studied using GEANT. The three configurations investigated were: 1) $42 \mathrm{~cm} \mathrm{~F} \mathrm{bG}+108 \mathrm{~cm}$ air gap +20 $\mathrm{m} \mathrm{Fe}$, 2) $42 \mathrm{~cm} \mathrm{PbG} \mathrm{+} \mathrm{no} \mathrm{air} \mathrm{gap} \mathrm{+} 20 \mathrm{~m} \mathrm{Fe}$, and 3) $42 \mathrm{~cm} \mathrm{PbG} \mathrm{+} \mathrm{no} \mathrm{air} \mathrm{gap} \mathrm{+} 20$ $\mathrm{m}$ marble $\left(2.78 \mathrm{~g} / \mathrm{cm}^{3}\right)$. The results for different momenta are given in Tables VII and VIII.

\begin{tabular}{|c|c|c|c|}
\hline $\begin{array}{c}\mathrm{p}_{\mu} \\
(\mathrm{GeV} / \mathrm{c})\end{array}$ & $\begin{array}{c}\text { PbG+air+Fe } \\
(\mathrm{cm})\end{array}$ & $\begin{array}{c}\text { PbG+Fe (no air gap) } \\
(\mathrm{cm})\end{array}$ & $\begin{array}{c}\text { PbG+marble (no air gap) } \\
(\mathrm{cm})\end{array}$ \\
\hline 1 & 12.0 & 6.2 & 10.9 \\
\hline 2 & 11.6 & 8.6 & 17.7 \\
\hline 3 & 12.6 & 11.4 & 21.0 \\
\hline 4 & 13.6 & 13.5 & 24.3 \\
\hline 5 & 15.8 & 15.0 & 27.3 \\
\hline
\end{tabular}

Table VII: RMS transverse beam size for stopped $\mu$ 's (from GEANT simulations).

\begin{tabular}{|c|c|c|}
\hline $\mathrm{p}_{\mu}$ & $\mathrm{PbG}+$ air $+\mathrm{Fe}$ & $\mathrm{PbG}+\mathrm{Fe}$ (no air gap) \\
\hline$(\mathrm{GeV} / \mathrm{c})$ & $(\mathrm{cm})$ & $(\mathrm{cm})$ \\
\hline 1 & 11.5 & 5.6 \\
\hline 2 & 5.5 & 2.4 \\
\hline 3 & 3.3 & 1.7 \\
\hline 4 & 2.6 & 1.3 \\
\hline 5 & 2.2 & 1.1 \\
\hline
\end{tabular}

Table VIII: RMS transverse beam size for $\mu$ 's at $1 \mathrm{GeV} / \mathrm{c}$ depth (from GEANT simulations).

These results indicate that any parallelism requirement must be loose if we are to retain acceptance for low-momenta $\mu$ 's using a detector that includes the $\mathrm{PbG}$ (with its accompanying space for PMT's and cables) as the electromagnetic calorimeter. 
Selected data from Sternheimer's straggling calculations for muons in $\mathrm{Cu}$ are shown in Table IX. The GEANT results, in Table X, are in reasonable agreement. The third column in Table $\mathrm{X}$ contains the half-width necessary for a symmetric cut to include $99 \%$ of the (1000) incident muons. (Because it sits on a long tail, this is a statistically shaky number that cannot be believed to better than $10 \%$ ). The final column gives the percentage of muons, from $\pi \rightarrow \mu \nu$ forced to decay at the front of the $\mathrm{PbG}$, that would pass the same cut that includes $99 \%$ of incident muons with the same momentum as the pion that is forced to decay.

\begin{tabular}{|c|c|}
\hline $\mathrm{p}_{\mu}(\mathrm{GeV} / \mathrm{c})$ & RMS straggling (percent) \\
\hline 1.13 & 3.150 \\
\hline 2.25 & 3.753 \\
\hline 3.38 & 4.218 \\
\hline 4.50 & 4.592 \\
\hline 6.76 & 5.163 \\
\hline 9.01 & 5.913 \\
\hline
\end{tabular}

Table IX: RMS straggling (from Sternheimer, Phys. Rev. 117, 485 (1960)).

\begin{tabular}{|c|c|c|c|}
\hline $\begin{array}{c}\mathrm{p}_{\mu} \\
(\mathrm{GeV} / \mathrm{c})\end{array}$ & $\begin{array}{c}\text { RMS straggling } \\
\text { (percent) }\end{array}$ & $\begin{array}{c}\text { \% cut to retain } \\
99 \% \text { of muons }\end{array}$ & $\begin{array}{c}\% \text { of } \pi \rightarrow \mu \nu \\
\text { passing } 99 \% \text { muon cut }\end{array}$ \\
\hline 1 & & \pm 5.8 & 18 \\
\hline 2 & 3.8 & \pm 6.4 & 17 \\
\hline 3 & 4.1 & \pm 8.0 & 22 \\
\hline 5 & 5.2 & \pm 10.7 & 38 \\
\hline 7 & 5.5 & \pm 11.5 & 44 \\
\hline
\end{tabular}

Table X: GEANT results for range straggling and $\pi \rightarrow \mu \nu$ rejection.

The above results confirm that we would benefit from a "range-resolution" that is less than that of the EXP791 rangefinder configuration (10\%), especially for lower-momentum muons. Therefore studies that will be discussed later assumed a detector that has gaps for instrumentation to provide $5 \%$ resolution.

\section{Design of Instrumented Muon Filter}

The instrumented iron design consists of four parts: how much iron to use, the size and segmentation of the MHO replacement, the position of additional timing counters in the MRG, and the position of MRG panels in the IMF.

A depth of $46 \mathrm{~cm}$ of iron (plus $42 \mathrm{~cm}$ of $\mathrm{PbG}$ ) is required to provide a hadronic filter while keeping high (99.8\%) efficiency for $1 \mathrm{GeV} / \mathrm{c}$ muons. The multiple 
scattering studies suggest that more iron might increase the efficiency of the MRG by decreasing the number of muons that escape through the sides before reaching the depth needed for a valid range measurement. GEANT simulations that studied this question used a sample of muons from EXP871 Monte Carlo $\mathrm{K}_{L} \rightarrow \mu e$ events that passed the parallelism and momentum cuts.

Some realistic measure of muon efficiency is needed since many of the muons that escape through the side of tine MRG have already reached a depth necessary for a valid range measurement. Instead of constructing a range table for each configuration, the ratio of (escape kinetic energy)/(incident total energy) is calculated. If the ratio is less than $10 \%$, then it is assumed that the muon would not fail the MRG cut. Typically half of the muons that escape have a ratio above $10 \%$.

Table XI contains the results for muon escape from the rangefinder for various configurations. The results for the number of total escaped muons and those with a ratio greater than $10 \%$ are given for designs with and without an air gap. After the first $46 \mathrm{~cm}$ of iron, gaps must exist in the iron to allow detectors for range measurements of muons with momenta in the range $1 \mathrm{GeV} / \mathrm{c}-1.5 \mathrm{GeV} / \mathrm{c}$. Thus, the next iron section is assumed to consist of eight 2 -inch Fe plates with a 2 -inch air gap between them $(80 \mathrm{~cm} .5 \mathrm{Fe})$. After that are sections that denoted as $88 \mathrm{~cm} .6$ $\mathrm{Fe}$ ( seven 3 inch Fe plates with 2 inch gaps) and $90 \mathrm{~cm} .66 \mathrm{Fe}$ (six 4 inch Fe plates with 2 inch gaps). This type of segmentation enables us to put detectors (MRG panels) in positions that give $4 \%$ to $6 \%$ range-resolution. All of the configurations listed, except for the last, assume that 1.5 inch thick Aluminum plates replace the air gaps between the marble plates of the MRG. For each case, the muons that escape out the downstream end of the MRG are not included. (Typically $0.2 \%$ $0.8 \%$ of the incident muons exit the downstream end of a 20 meter MRG). These results confirm our expectation that the muon detection efficiency is increased by using more iron and by minimizing the number of air gaps.

\begin{tabular}{|c|c|c|c|c|}
\hline configuration & $\begin{array}{c}\text { total } \\
\text { (with air gap) }\end{array}$ & $\begin{array}{c}\mathrm{KE} / \mathrm{E}_{\text {in }}>.1 \\
\text { (with air gap) }\end{array}$ & $\begin{array}{c}\text { total } \\
\text { (no air gap) }\end{array}$ & $\begin{array}{c}\mathrm{KE} / \mathrm{E}_{\text {in }}>.1 \\
\text { (no air gap) }\end{array}$ \\
\hline $46 \mathrm{~cm} \mathrm{Fe}$ & $7.75 \pm 0.44$ & $4.51 \pm 0.34$ & $5.80 \pm 0.26$ & $3.11 \pm 0.19$ \\
\hline$+80 \mathrm{~cm} .5 \mathrm{Fe}$ & $7.60 \pm 0.43$ & $4.18 \pm 0.32$ & $6.13 \pm 0.27$ & $2.92 \pm 0.19$ \\
\hline$+88 \mathrm{~cm} .6 \mathrm{Fe}$ & $5.66 \pm 0.35$ & $2.84 \pm 0.25$ & $5.12 \pm 0.25$ & $2.47 \pm 0.17$ \\
\hline$+90 \mathrm{~cm} .66 \mathrm{Fe}$ & $4.92 \pm 0.24$ & $2.51 \pm 0.17$ & $4.01 \pm 0.22$ & $1.74 \pm 0.14$ \\
\hline $\begin{array}{c}\text { same but no } \\
\text { Al between marble }\end{array}$ & $7.19 \pm 0.29$ & $3.38 \pm 0.20$ & & \\
\hline
\end{tabular}

Table XI: GEANT results for muon inefficiency (in percent) due to escapes out the side for different configurations. Configurations with ".5 $\mathrm{Fe}$ " refer to Fe plates spaced to accommodate detectors in such a way that the average density is 0.5 the density of iron. 
Choice of $\mathrm{z}$ position for the new MHO detector is straightforward once the electromagnetic calorimeter upstream is determined. The transverse segmentation also depends upon the upstream detector because of different multiple scattering effects for different designs. In addition, the transverse segmentation must be consistent with the parallelism logic. We have been requested to have a number consistent with the segmentation in the Cerenkov counter which will be 8 per side. This implies 8 scintillator slats $/$ side, each $13.5 \mathrm{~cm}$ wide. The length of these scintillators will be approximately $2.5 \mathrm{~m}$ based on the spread of low-momentum muons which traverse $46 \mathrm{~cm}$ of iron. The final size depends on the final detector configuration.

The parallelism logic could form an 'OR' of three slats or five slats. The multiple scattering studies indicate that to have $99 \%$ efficiency for low-momentum muons we need a transverse extent of $\pm 2.5 \times \sigma$, or nearly $60 \mathrm{~cm}$ for the configuration with the $\mathrm{PbG}$ and air gap. This means that we need to 'OR' five slats together and therefore can only effectively suppress $3 / 8$ of the accidental muon triggers. An 'OR' of three slats loses about $10 \%$ of $1 \mathrm{GeV} / \mathrm{c}$ muons but retains almost all of the muons above $2 \mathrm{GeV} / \mathrm{c}$. The segmentation of the $\mathrm{y}$-counters have no constraints from the parallelism logic. However, they must be segmented enough that rates are not a problem. The likely dimensions are $108 \mathrm{~cm}$ long $\times 25 \mathrm{~cm}$ wide $\times 1.27$ $\mathrm{cm}$ thick. A total of 10 counters per side is needed.

Additional scintillation counters downstream of the $\mathrm{MHO}$ can reduce the shortcomings of the MRG panels. These shortcomings are 1) accidentals can be a problem because all that is required is a latch in a 160 ns time window, and 2) the $y$-measuring counters extend over the entire x-extent of the detector (both sides). Use of a fast-timing detector, with the $y$-counter split at $x=0$, can solve both of these problems. It is hard to be quantitative about the benefits of such a detector because the main advantage is to increase the confidence in a given event. We could gain one percent, or so, in muon efficiency since a poor time in the MHO need not be rejected automatically if there are other measures of time. Two possible positions for additional scintillation planes are given in Table XII. The number and size of these scintillator slats would probably be the same as the MHO-replacement scintillators, unless EXP791 MHO scintillators are reused. We believe this is a possible option that should be explored.

In addition to the three scintillator planes, we estimate that $19 \mathrm{MRG}$ panels will be necessary to obtain $5 \% \Delta p / p$ resolution in the range of 1.0 to $2.8 \mathrm{GeV}$, which we propose to cover with iron. We expect these detectors to remain the responsibility of the William and Mary group. However, the mechanical supports that are required to position the MRG detectors between the iron plates will be the responsibility of our group. Fig. 4 shows our proposed design. 


\begin{tabular}{|c|c|c|c|}
\hline $\begin{array}{c}\mathrm{p}_{\mu} \\
(\mathrm{GeV} / \mathrm{c})\end{array}$ & $\begin{array}{c}\text { amount of iron } \\
(\mathrm{cm})\end{array}$ & $\begin{array}{c}\text { muon efficiency } \\
\text { (percent) }\end{array}$ & $\begin{array}{c}\text { pion induced counts } \\
\text { (percent) }\end{array}$ \\
\hline 1 & 46 & 99.8 & 7.0 \\
\hline 1.5 & $46+" 80 "$ & 91 & 1.0 \\
\hline 2.1 & $46+" 80 "+" 88 "$ & 71 & 0.4 \\
\hline
\end{tabular}

Table XII: The percentage of muons from parallel $K_{L} \rightarrow \mu e$ that reach possible positions for an MRG timing counter given in units of momentum. The percentage of pion punchthrough (from parallel $\mathrm{K}_{e 3}$ ) is also given.

\section{References}

[1] H. Haber and G. L. Kane, Phys. Rep. 117, 75(1985); B. A. Campbell, Phys. Rev. D28, 209(1983); J. C. Romao et al., Nucl. Phys. B250, 295(1985).

[2] E. Farhi and L. Susskind, Phys. Rep. 74, 277(1981); E. Eichten and K. Lane, Phys. Lett. 90B, 125(1980); J. Ellis et al., Nucl. Phys. B182, 529(1981); A. Masiero, E. Papantonopoulos, and T. Yanagida, Phys. Lett. 115B, 229(1982); B. Holdom, Phys. Lett. 143B, 227(1984); E. Eichten et al., Phys. Rev. D34, 1547(1986).

[3] J. C. Pati and A. Salam, Phys. Rev. D10, 275(1974); A. Barroso, G. C. Branco, and M. C. Bento, Phys. Lett. 134B, 123(1984).

[4] T. Maehara and T. Yanagida, Prog. Theo. Phys. 60, 1434(1979); A. Davidson and K. C. Wali, Phys. Rev. Lett. 26, 691(1981); Wei-Shu Hou and A. Soni, Phys. Rev. Lett. 54, 2083(1985).

[5] R. N. Cahn and H. Harari, Nucl. Phys. B176, 135(1980).

[6] Z. Y. Zhu, Z. Phys. C13, 321(1982); I. Bars, M. J. Bowick, and K. Freese, Phys. Lett. 138B, 159(1984); O. W. Greenberg, R. N. Mohapatra, and S. Nussinov, Phys. Lett. 148B, 465(1984); J. C. Pati, Phys. Rev. D30, 1144(1984).

[7] B. A. Campbell et al., Int. J. Mod. Phys. A2, 831(1987).

[8] J. L. Ritchie, invited talk at the Topical Conference of the SLAC Summer Institute, July, 1990; W. R. Molzon, invited talk at TRIUMF workshop, UCI90-52, July, 1990; W. R. Molzon, invited talk at the XXV International Conference on High Energy Physics, Singapore, August, 1990.

[9] L. M. Sehgal, Phys. Rev. 183, 1511(1969); B. R. Martin, E. de Rafael and J. Smith, Phys. Rev D2, 179(1970). 
[10] T. Akagi, et al., Phys. Rev. Lett. 67, 2614 (1991).

[11] A.P. Heinson, et al., Phys. Rev. D44, R1 (1991).

[12] J. Frank et. al., IEEE Trans. in Nucl. Science 36, 79(1989).

\section{Figure Captions}

Figure 1: Plan view of the EXP791 detector.

Figure 2: Plan view of the EXP871 detector.

Figure 3: Horizontal cross section view of the optimized beam plug used in the 1991 test for EXP871.

Figure 4: Horizontal cross section view of the instrumented muon filter proposed for EXP871.

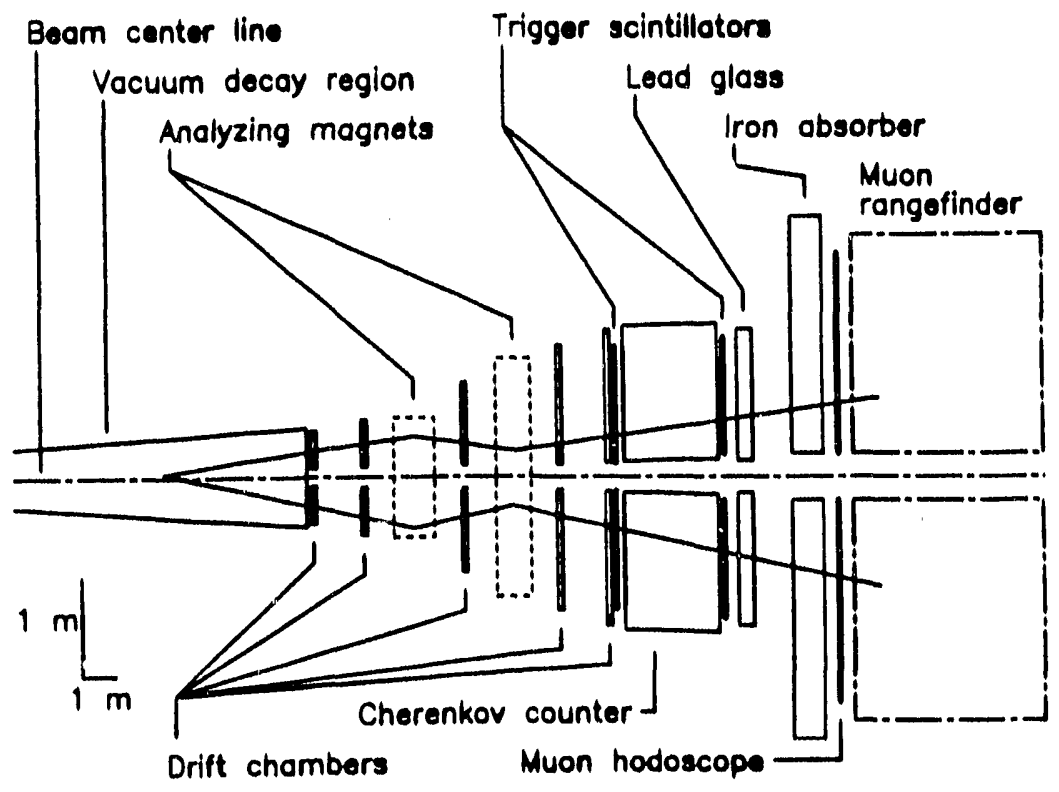

Figure 1 


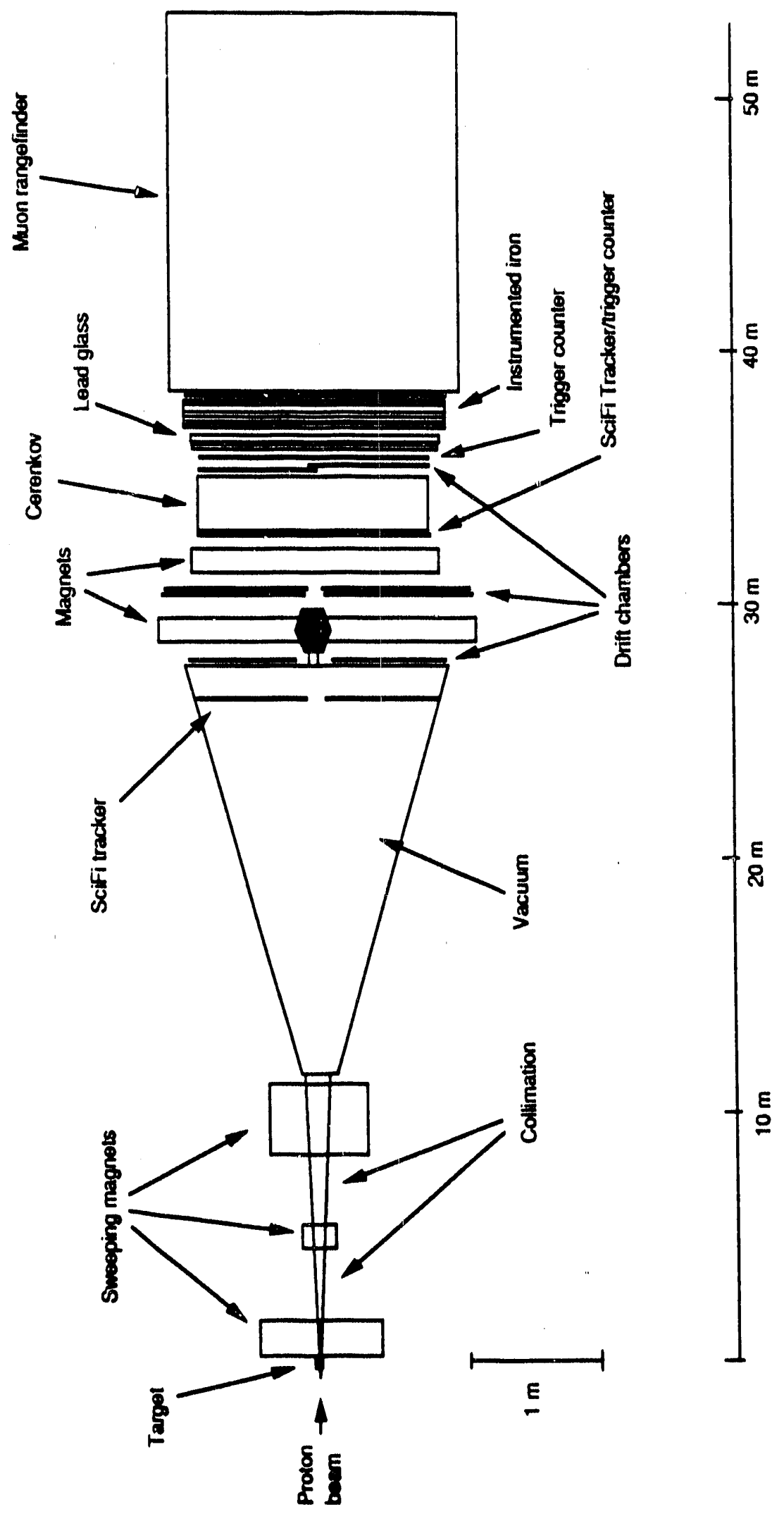

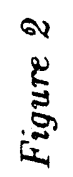




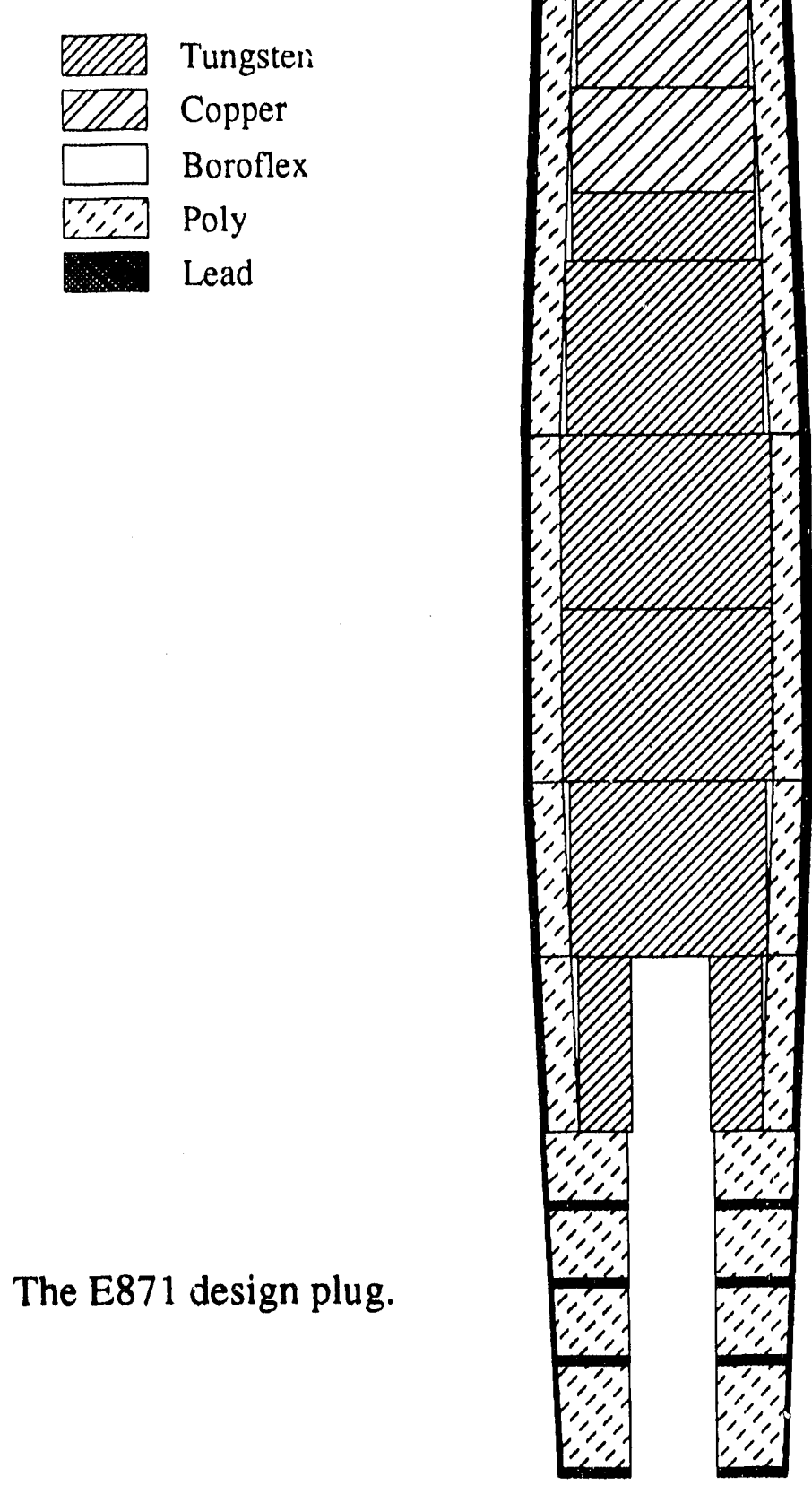

Figure 3 
Pb Glass

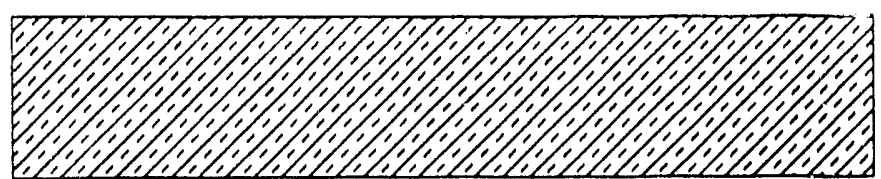

Z7 Pb Glass

$\square$ Iron

E'] Scintillator

MRG Pane!

(Z) MRG

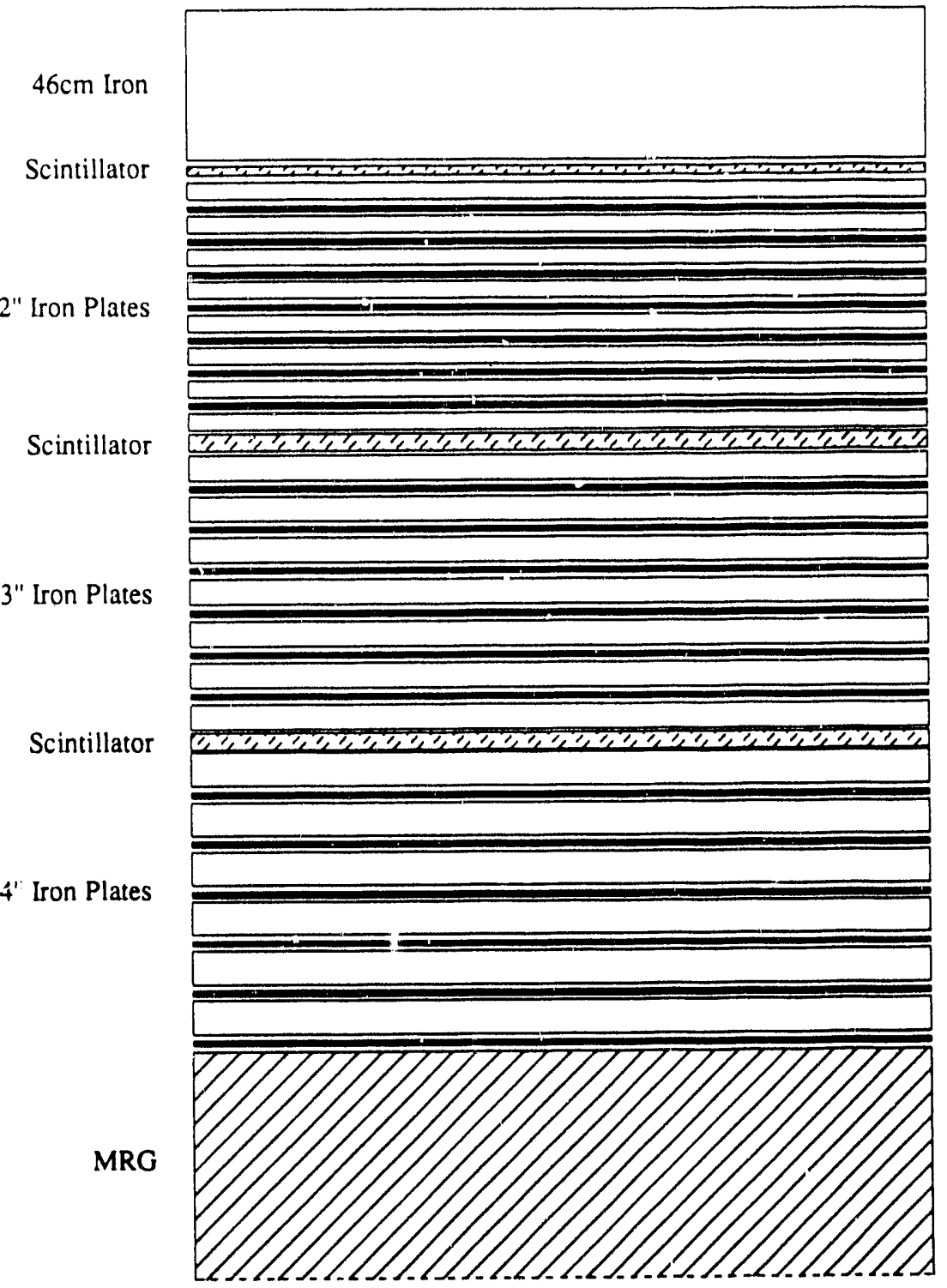




\section{New Experimental Projects}

The experiments discussed in this section have been approved by the LAMPF Program Advisory Committee (PAC). We anticipate that 1 or 2 of these experiments will be scheduled for production runs during 1992-1993.

\subsection{EXP1040: Spin Variable Measurements of the $\left(p, r_{i}\right)$ Inclusive Reaction on Several Nuclei}

(G. W. Hoffmann, L. Ray, D. Read, S. Worm;

IUCF; LANL; Colorado; OSU; Ohio Univ.; C. D. Goodman,

G. W. Hoffmann, T. N. Taddeucci, spokesmen)

Summary: The Neutron Time-of-Flight facility (NTOF) at LAMPF will be used to measure polarization transfer $\left(D_{n n}\right)$ for inclusive $(p, n)$ on ${ }^{1} \mathrm{H},{ }^{2} \mathrm{H},{ }^{12} \mathrm{C},{ }^{40} \mathrm{Ca}$, and ${ }^{208} \mathrm{~Pb}$ at $0^{\circ}$ and $6^{\circ}$ at $800 \mathrm{MeV}$. The energy loss range from the isobaric analogue state (IAS) and Gamow-Teller (GT) peaks through the region of quasielastic $\Delta$ production will be examined. These data will provide the first comprehensive survey of GT excitation strength in nuclei for energy losses including the $\Delta$ region. Analyzing powers from $6^{\circ}-30^{\circ}$ in $6^{\circ}$ steps at $800 \mathrm{MeV}$ for the quasielastic and $\Delta$ regions will also be measured. These data will complement existing inclusive $\left(p, p^{\prime}\right)$, $\left(p, n^{\prime}\right),\left({ }^{3} \mathrm{He}, t\right),(d, 2 p),\left(e, e^{\prime}\right)$, and photo-absorption data which exhibit the same two-body excitations. Theoretical work is presently underway in an attempt to understand the positions and widths of the quasielastic and $\Delta$ peaks for the various reactions. The experiment will provide information concerning: (1) the isovector response of the nucleus, (2) the $\Delta$-nucleon interaction in the nuclear medium, (3) medium modifications to the isovector part of the $\mathrm{NN}$ effective interaction, and (4) requirements for $\mathrm{NN}$ inelastic models.

\section{Quasifree $\triangle$ Production}

For proton-proton scattering at $800 \mathrm{MeV}$ laboratory incident energy, the $\Delta(1232)$ resonance dominates the inclusive energy-loss spectra for both inelastic scattering and for $(p, n)$ charge exchange. In this context excitation of the $\Delta$ can be thought of as a fundamental excitation of the nucleon. The same resonance dominates the charge exchange spectra for $p\left({ }^{3} \mathrm{He}, t\right)$ and $p(d, 2 p)$. For nuclear targets the charge exchange spectra for these three reactions show three distinct regions. At small energy loss the spectrum is dominated by Fermi (IAS) and Gamow-Teller transitions. At larger energy loss quasi-free knockout of nucleons is discernible, and at 
yet larger energy loss the $\Delta(1232)$ resonance dominates the spectra.

We will determine the spin observables to enhance our knowledge of the process of $\Delta$ excitation, for the fundamental nucleon-nucleon process, as well as the nucleon-nucleus process. The only existing spin-dependent measurements for the $\Delta$ region for nuclear targets are analyzing power data for $\left(\vec{p}, p^{\prime}\right)$; the interpretation of which is ambiguous owing to the large $\Delta$ decay background $(\Delta \rightarrow \pi+N)$ in the kinematic region of the two-body $p+N \rightarrow N+\Delta$ direct quasifree process. Even so the $800 \mathrm{MeV} \vec{p}+{ }^{12} \mathrm{C}$ inclusive analyzing power data ${ }^{1}$ show very interesting structure centered about the direct $\Delta$ production peak. Theoretical calculations have been made for the inclusive $(p, n)$ reaction that show sensitivity of the predicted spin observables to the dynamical ingredients in the calculations.

In $(p, n),\left({ }^{3} \mathrm{He}, t\right)$ and $(d, 2 p)$ reactions, a shift in the apparent position of the $\Delta$ resunance peak in the energy loss spectra is seen between the data with the proton target and the data with nuclear targets. Part of the shift is due to Fermi motion and reaction kinematics. These account for about $40 \mathrm{MeV}$ of the shift, leaving $30 \mathrm{MeV}$ unexplained. This latter part of the shift is presumed to be due to a nuclear medium correlation effect on the spin-longitudinal response function. ${ }^{2}$ Recent calculations ${ }^{3,4}$ suggest that this residual $30 \mathrm{MeV}$ energy shift may be caused by the energy-dependent $\pi$ exchange interaction in the nuclear medium. The $\pi$ exchange provides a strongly attractive interaction in the spin-longitudinal $(\vec{\sigma} \cdot \vec{q})$ channel at these momentum transfers leading to a lowering of the $\Delta$ mass produced in the target. No significant shift occurs in the spin- transverse $(\vec{\sigma} \times \vec{q})$ channel, since here the interaction is weakly repulsive.

A new calculation for the ${ }^{12} \mathrm{C}(p, n)$ reaction by Oltmanns, Osterfeld, and Udagawa ${ }^{5}$ does a reasonable job in fitting the experimental spectrum near the peak of the $\Delta$ resonance (see Fig. 1). This model predicts a strong dependence of the $0^{\circ}$ spin transfer coefficients $D_{n r}\left(=C_{x x}\right)$ and $D_{l l}\left(=C_{z z}\right)$ on the energy transfer (see Figs. 2-3). The spin observables are strongly dependent on the $\Delta$-nucleon hole effective interaction. The proposed experiment should produce data that can be compared directly with this prediction.

Bohr and Mottelson ${ }^{6}$ suggested that the $\Delta$ plays a crucial role in the missing GT strength problem. In their model $\Delta$ excitation is part of the total GT strength function. Measurement of $D_{n n}$ in charge-exchange provides a reliable tool for experimentally finding GT strength in nuclear excitalion spectra since the spin-flip probability $\left(S_{F P}\right)$ is measured directly, where $S_{F P}=\frac{1}{2}\left(1-D_{n n}\right)$. At low energies $S_{F P}$ in charge-exchange is determined by the $t_{\sigma \tau}$ component of the NN force and $D_{n n}=-\frac{1}{3}$ for GT transitions at zero degrees. At higher energies important isovector-tensor terms also contribute, but $S_{F P}$ remains a good indicator of GT strength.

The spin observable $D_{n n}$ is easily inte.pretable in certain situations. Consider, for example, three regions of the $(p, n)$ spectrum. In the bound state region (small energy loss) the value of $D_{n n}$ is controlled by the structure of the nuclear states. Thus, at zero degrees the structure of the IAS restricts the interaction to $\left(\vec{\tau}_{1} \cdot \vec{\tau}_{2}\right)$ and 
$D_{n n}$ has the value +1 . For a GT transition the structure restricts the interaction to $\left(\vec{\sigma}_{1} \cdot \vec{\sigma}_{2}\right)\left(\vec{\tau}_{1} \cdot \vec{\tau}_{2}\right)$ (if no tensor terms are important), and $D_{n n}=-\frac{1}{3}$. For quasi-free scattering, in the absence of medium modifications, $D_{n n}$ would be expected to have the free $\mathrm{N}-\mathrm{N}$ value which is energy dependent. For the $\Delta$, we really don't know exactly what to expect. We do expect the coupling to be dominated by $\pi$ and $\rho$ exchange. In the usual separation of the one-pion exchange potential, it contains a central $\left(\vec{\sigma}_{1} \cdot \vec{\sigma}_{2}\right)\left(\vec{\tau}_{1} \cdot \vec{\tau}_{2}\right)$ term and a tensor term. The central term alone at zero degrees yields $D_{n n}=D_{l l}=-\frac{1}{3}$ but tensor terms can alter this value.

In any case, the $\Delta$ is an essential component of nuclear physics, and the coupling between the nucleon and the $\Delta$ is of fundamental importance, both in free space and in the nuclear medium. The $\Delta$-nucleus potential is also of fundamental importance and is an essential ingredient in models of pion-nucleus scattering. Spin observables are necessary to constrain the $N-\Delta$ amplitudes and very few data are available at present.

\section{Quasielastic $(p, n)$}

In the quasi-free region, $D_{n n}$ is not constrained by the quantum numbers of specific nuclear states. We expect it to differ from the free $\mathrm{N}-\mathrm{N}$ scattering value due to medium modifications and/or relativistic effects.

The quasi-free region has been studied in charge exchange with the $\left({ }^{3} \mathrm{He}, t\right)$ reaction. Bergqvist et al. ${ }^{7}$ compare the centroids of the quasi-free peak in $\left({ }^{3} \mathrm{He}, t\right)$ and $\left(e, e^{\prime}\right)$ reactions. In an $\omega$ versus $q$ plot the two reactions match below $q=1.5 \mathrm{fm}^{-1}$ but depart at higher momentum transfers. Alberico et al. ${ }^{8}$ attempt to connect this shift with a softening of the longitudinal response. However, Carey et al. ${ }^{9}$ found that the ratio of transverse to longitudinal response in $\left(p, p^{\prime}\right)$ was essentially unity, implying no significant medium corrections to the spin longitudinal or transverse response. The latter experiment included both isoscalar and isovector channels. It is important to try to clarify the picture with spin data from a pure isovector probe.

Measurements of the analyzing power for quasifree ${ }^{12} \mathrm{C}(p, n)$ at $E_{p}=494 \mathrm{MeV}$ are in good qualitative agreement with predictions, ${ }^{10}$ showing a slight enhancement with respect to the free values. Interestingly, this apparent agreement does not hold at higher energies. At $789 \mathrm{MeV}$ the analyzing power at several angles measured in Cycle 55 is smaller than the predicted and free NN values. This could signal either a flaw in the model or a change in reaction mechanism. New data at several angles for several targets will help to sort out both mass and momentum transfer dependence effects.

\section{Experimental Details}

We plan to use the NTOF detector/polarimeter with a $200 \mathrm{~m}$ flight path. A micropulse spacing of $400 \mathrm{~ns}$ will be required to avoid contamination of the spectra with $\gamma$-rays from $\pi^{0}$ production. Energy loss of up to $500 \mathrm{MeV}$ will be contained within this time window. $\mathrm{CH}_{2}, \mathrm{CD}_{2}$, and $\mathrm{C}$ targets will be used to 
obtain the ${ }^{1} \mathrm{H}$ and ${ }^{2} \mathrm{H}$ data. This technique has been successfully employed in the spin transfer measurements for E881 and avoids the safety hazards and other technical difficulties associated with cryogenic hydrogen targets.

\section{References}

[1] J. A. McGill et al., Phys. Lett. B 134, 157 (1984).

[2] G. Chanfray and M. Ericson, Phys. Lett. B 141, 163 (1984).

[3] J. Delorme and P. A. M. Guichon, Proc. of the 10th Biennale de Physique Nucleaire, p. C.4.1, Aussois, 1989, Lycen 8906; Phys. Lett. B 263, 157 (1991).

[4] T. Udagawa, S. W. Hong, and F. Osterfeld, Phys. Lett. B 245, 1 (1990).

[5] P. Oltmanns, F. Osterfeld, and T. Udagawa, to be published.

[6] A. Bohr and B. Mottelson, Phys. Lett. B 100, 10 (1981).

[7] I. Bergqvist et al., Nucl. Phys. A469, 648 (1987).

[8] W. M. Alberico et al., Phys. Rev. C 38, 109 (1988).

[9] T. A. Carey et al., Phys. Rev. Lett. 53, 144 (1984).

[10] C.J. Horowitz and D.P. Murdock, Phys. Rev. C 37, 2032 (1988).

\section{Figure Captions}

Figure 1: (a) Comparison of data and calculations for the quasifree $\Delta$ excitation in ${ }^{12} \mathrm{C}(p, n)$ at $800 \mathrm{MeV}$ and $\theta=0^{\circ}$ (from Ref. 4). (b) Comparison of data and calculations for the quasifree $\Delta$ excitation in ${ }^{12} \mathrm{C}(p, n)$ at $800 \mathrm{MeV}$ and $\theta_{\text {lab }}=9^{\circ}$ (from Ref. 5).

Figure 2: Predictions for the polarization transfer coefficient $D_{n n}\left(C_{x x}\right)$ for the $\Delta$-excitation region in ${ }^{12} \mathrm{C}(p, n)$ at $800 \mathrm{MeV}$ and $\theta=0^{\circ}$. Calculations including and not including the $\Delta$-nucleon hole interaction are shown by the solid and dashed lines, respectively.

Figure 3: Predictions for the polarization transfer coefficient $D_{\ell \ell}\left(C_{z z}\right)$ for the $\Delta$-excitation region in ${ }^{12} \mathrm{C}(p, n)$ at $800 \mathrm{MeV}$ and $\theta=0^{\circ}$. Calculations including and not including the $\Delta$-nucleon hole interaction are shown by the solid and dashed lines, respectively. 


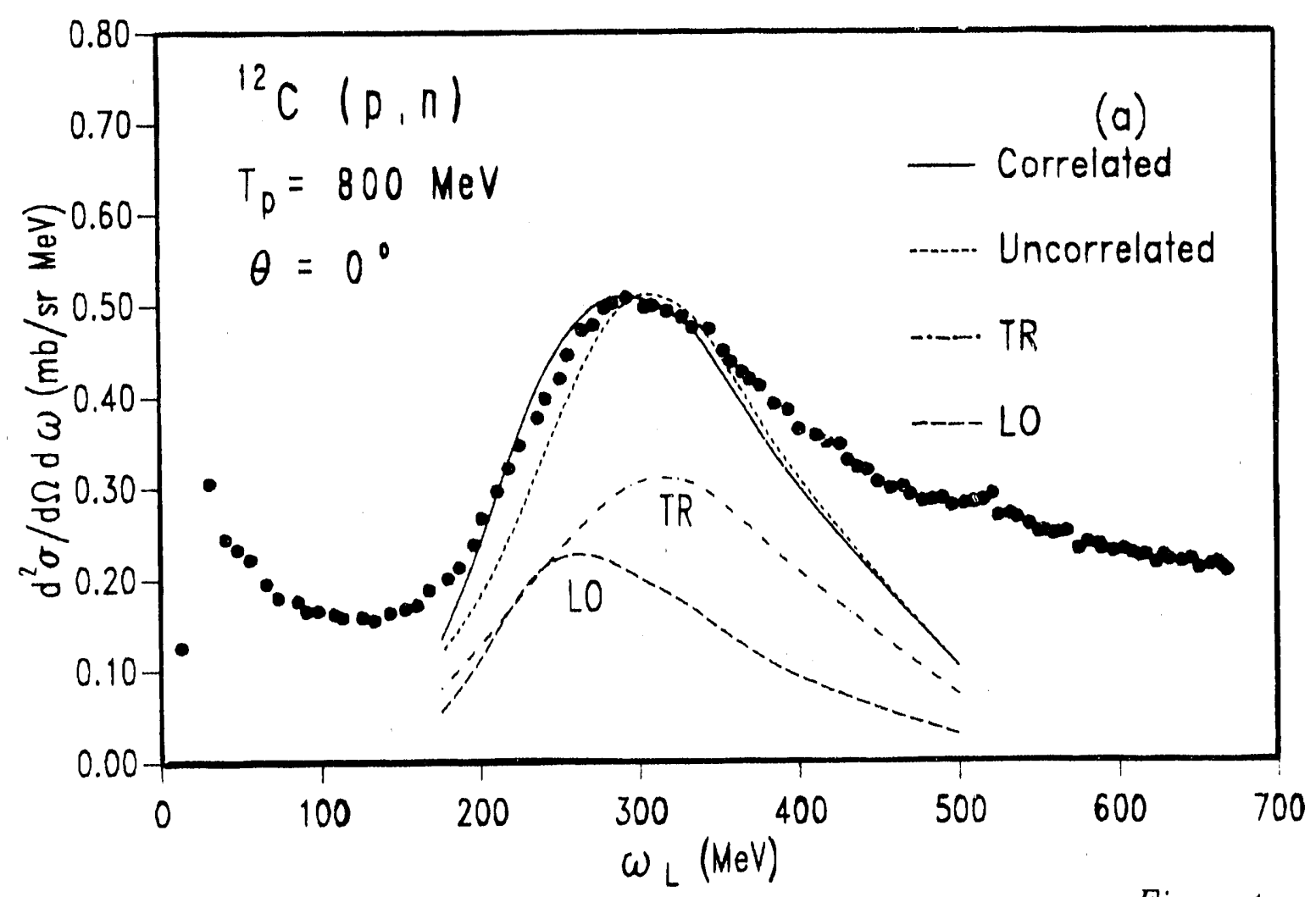

Figure 1a

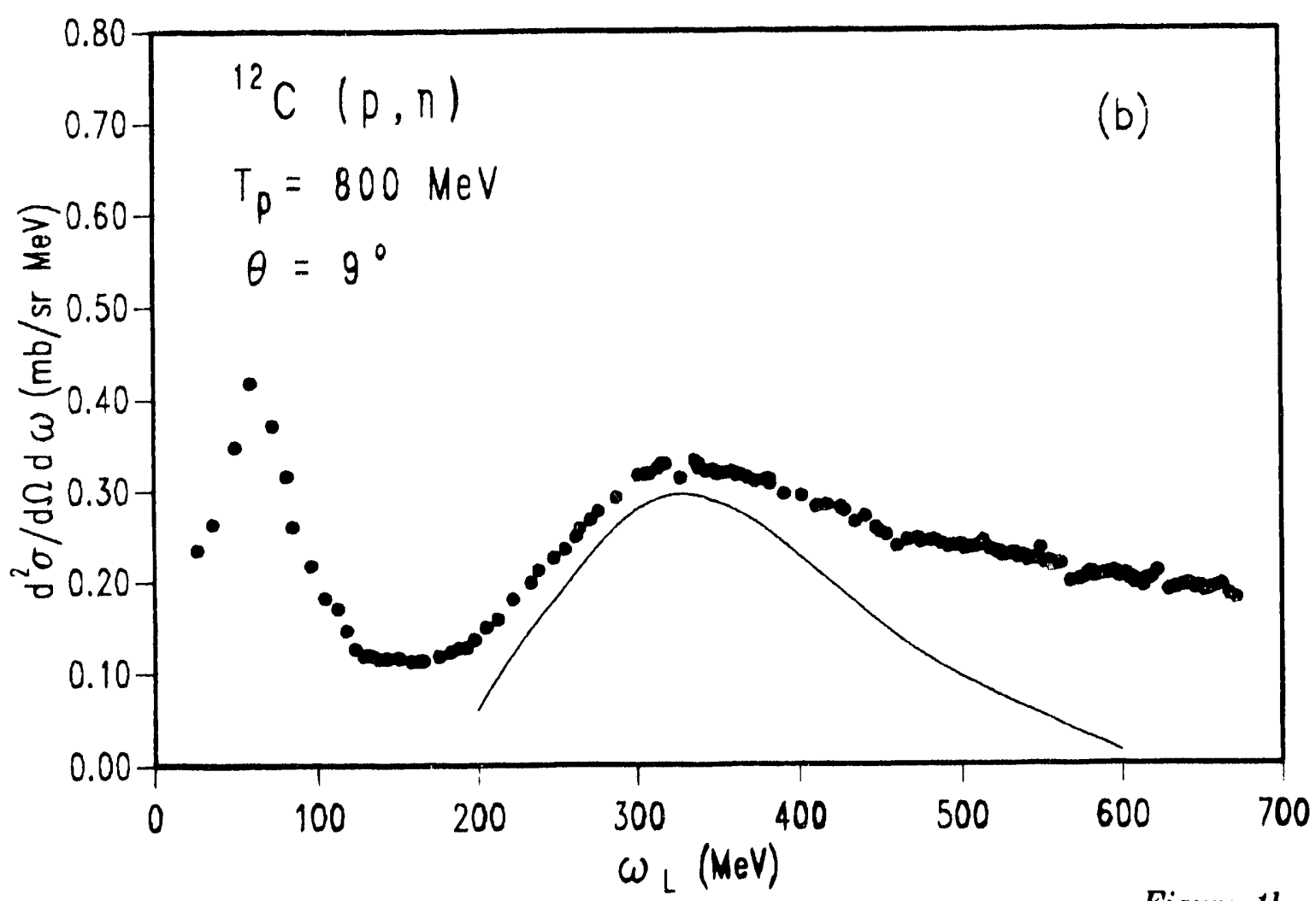

Figure $1 b$ 


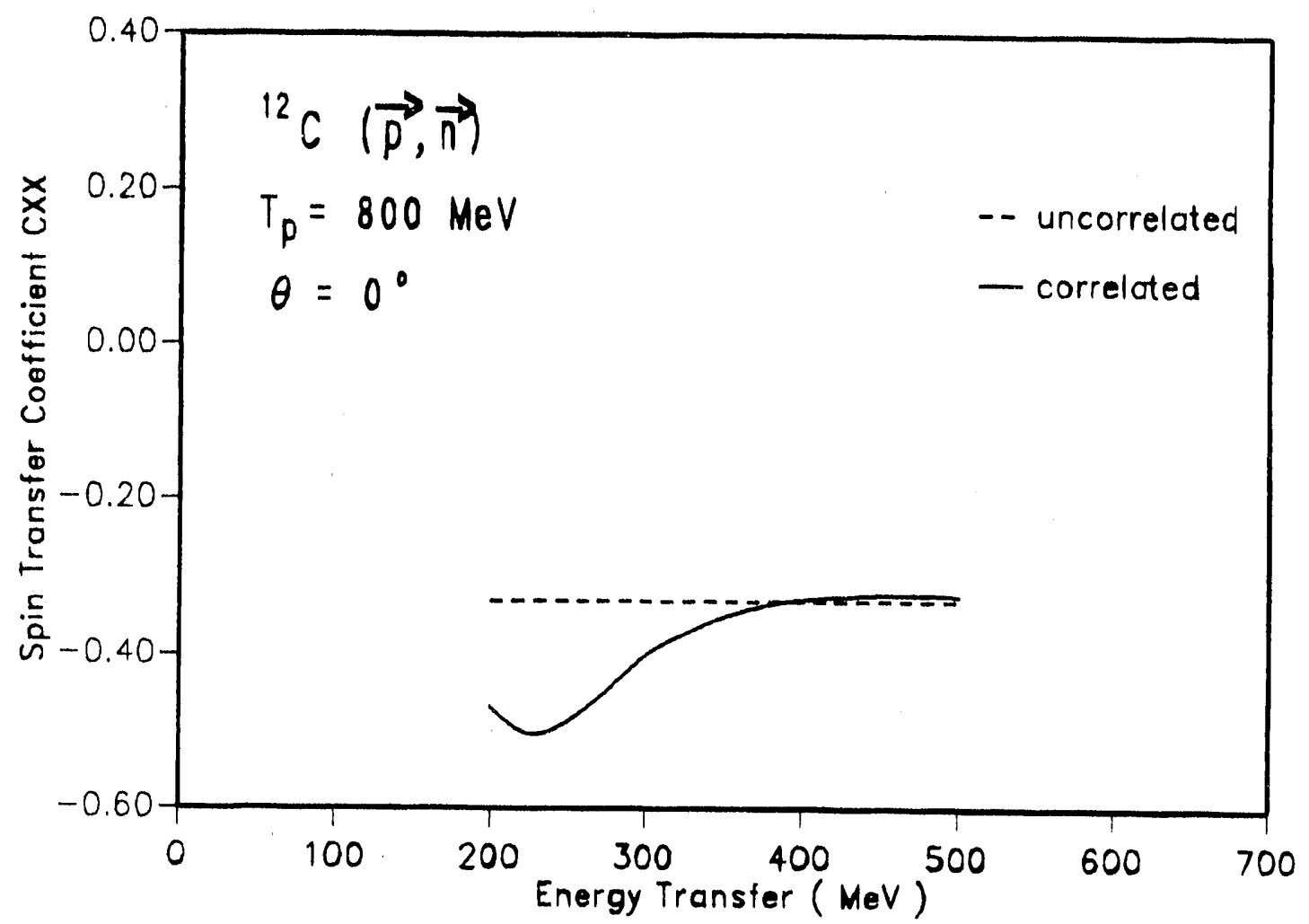

Figure 2

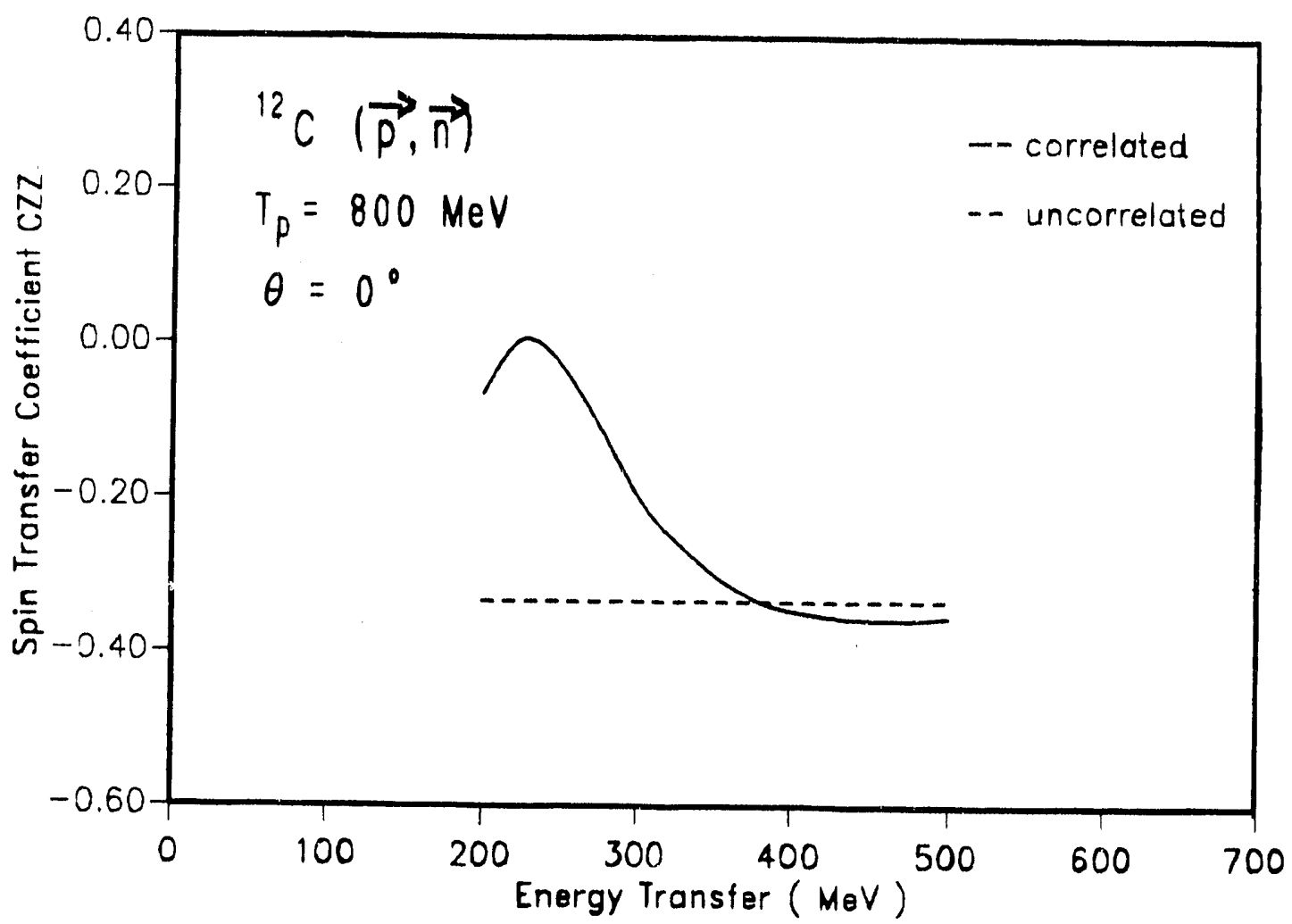

Figure 3 


\subsection{EXP1097: Single Pion Production in $n p$ Scattering}

(G. W. Hoffmann, L. Ray; Texas; ANL; UCLA; U. Hous`nn; JPL;

LANL; Manitoba; Queen Mary College; Rice; TAMU;

N. E. Davison, G. Mutchler, D. L. Adams, P. J. Riley, spokesmen)

Summary: Medium energy differential cross sections, analyzing powers and spin correlation parameters for the $n p \rightarrow p p \pi^{-}$reaction will be measured. A polarized neutron beam $(P=0.4)$ with energy from $500-800 \mathrm{MeV}$ will be scattered from an unpolarized $\mathrm{LH}_{2}$ target (Phase I), and from a longitudinally polarized, frozen spin, hydrogen target (Phase II). All three forward going charged reaction products will be detected in a large solid angle detector with multiple track capability consisting oi a cylindrical ćrift chamber and an array of vertical scintillator bars. Measurements will be made with $\hat{s}, \hat{n}$, and $\hat{\ell}$-type polarized neutron beams. In Phase I differential cross section, $A_{o o s o}, A_{o o n o}$, and $A_{o o l o}$ will be obtained. In Phase II the

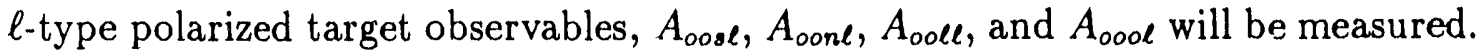
These data will complement existing $\mathrm{NN} \rightarrow \mathrm{NN} \pi$ data and will provide new information on the $\mathrm{I}($ isospin $)=0$ channel and greater sensitivity to "nonresonant" $\pi \mathrm{N}$ channels. These data should make possible a phase shift analysis of the $I=0$ inelastic amplitudes.

The motivation for studying $\mathrm{NN} \rightarrow \mathrm{NN} \pi$ relates to (1) understanding nuclear structure and reactions since knowledge of the pion and delta degrees of freedom is needed for such studies, (2) searches for possible subnucleonic degrees of freedom, such as dibaryon resonances, and (3) tests of models of the off-shell $\pi \mathrm{N}$ amplitudes.

The reactions $\mathrm{NN} \rightarrow \mathrm{NN} \pi$ occur through several isospin channels. If these are labelled by the initial total isospin, $I$, and the final two-nucleon total isospin, $\mathrm{I}^{\prime}$, the allowed channels are for $\left(I, I^{\prime}\right):(1,1),(1,0)$, and $(0,1)$. The only extensively studied reaction so far is $p p \rightarrow n p \pi^{+}$which involves the $(1,0)$ and $(1,1)$ channels. Data also exist for the closely related $p p \rightarrow d \pi^{+}$reaction which is the $(1,0)$ channel. The reaction to be studied here, $n p \rightarrow p p \pi^{-}$, occurs through the $(1,1)$ and $(0,1)$ channels. Unlike the earlier reactions, this one is sensitive to the $I=0$ channel $(0,1)$, the dominant $(1,0)$ channel being excluded.

Although all $\mathrm{NN} \rightarrow \mathrm{NN \pi}$ reactions are dominated at medium energies by intermediate $\Delta$ production, nonresonant amplitudes may also be important, particularly for the spin observables. The study of $n p$ pion production presents an opportunity to study these nonresonant amplitudes because they are expected to be relatively stronger in this reaction than for $p p$ pion production (due to the absence of the $\Delta^{++}$intermediate state).

There have been only two published studies of the $n p \rightarrow p p \pi^{-}$reaction with good statistics; one between 500 and $600 \mathrm{MeV}$ (Ref. 1) and one at $795 \mathrm{MeV}$ (Ref. 2). Neither reaction was kinematically complete and neither measured spin correlation 
parameters. Differential cross section and analyzing power data at limited angles have been taken but are unpublished. ${ }^{3}$ Measurements of the $n p \rightarrow p p \pi^{-}$differential cross section and analyzing powers have been made at TRIUMF at $440 \mathrm{MeV}$ (Ref. 4) and at SATURNE at $650 \mathrm{MeV}$. In EXP1097 we will cover a broader range of kinematics, span energies from 500 to $800 \mathrm{MeV}$, and will measure spin correlation parameters using a polarized hydrogen target.

The detector system, shown in Fig. 1, consists of a large array of scintillator bars surrounding a cylindrical drift chamber; the target is at the center. The vertical angular acceptance varies from $-60^{\circ}$ to $+60^{\circ}$ while the horizontal acceptance varies from $-120^{\circ}$ to $+120^{\circ}$ for Phase II (larger for Phase I). The scintillator bars provide the first level trigger and will measure the time-of-flight for the outgoing protons. The cylindrical drift chamber will be able to detect all three charged particles for more than $50 \%$ of the phase space. Positions perpendicular to the sense wires are determined by measuring the drift time. Vertical positions along the sense wires are determined using charge division.

The drift chamber is a $1.1 \mathrm{~m}$ long cylinder of $16.4 \mathrm{~cm}$ inner and $54 \mathrm{~cm}$ outer diameter with 1792 high voltage (HV) and 448 sense wires. The wires are arranged in 20 concentric rings consisting of four groups of five rings of wires each as shown in Fig. 2. The HV wires and the sense wires are arranged in a hexagonal pattern as shown in Fig. 2.

During 1991 a portion of the cylindrical drift chamber was tested at LAMPF in Area B downstream of the EXP876 setup. One-fourth of the sense wires were instrumented and used to detect and track recoil protons from $n+p$ scattering in the $\mathrm{LH}_{2}$ target. The test run successfully demonstrated the operation and expected spatial resolution of the chamber. Further testing of the drift chamber and associated electronics is planned for summer 1992.

For Phase II a frozen-spin polarized target will be used as shown in Fig. 3. The R\&A magnet is being tested at LAMPF and measurements of the field strength at the holding position of the target will be made soon. Tests of the performance of the drift chamber in the presence of the holding field of the magnet will also be conducted. The dilution refrigerator is being modified in order to lower the base temperature to $50 \mathrm{mK}$ or less in order to maintain target polarization in the holding field. The insert of the refrigerator will be modified to gain a large diameter, low mass access for the neutron beam. Possible target materials include chemically doped 1-butanol and irradiated ammonia.

A major part of the data analysis involves determining the five kinematic parameters which characterize a three-body final state. The drift chambers will determine the directions of the three particles (6 quantities) and the scintillator bars the velocities of the two forward going protons. Thus the data will overdetermine the three-body kinematics and chi-square fitting will be used to reconstruct events and reject background. High quality event reconstruction is particularly important for Phase II where large backgrounds are expected due to quasi-free scattering from protons in the nuclei of the target material. 
A successful microscopic description of the $\mathrm{NN} \rightarrow \mathrm{NN} \pi$ reaction has not yet been given. The only model for which extensive predictions exist is the relativistic threebody model of Dubach, Kloet, and Silbar (DKS). ${ }^{5}$ This model has as input the $\pi \mathrm{N}$ interactions in the $\mathrm{P}_{11}$ and $\mathrm{P}_{33}$ channels, and predicts amplitudes $\mathrm{T}(\mathrm{NN} \rightarrow \mathrm{NN})$ and $\mathrm{T}(\mathrm{NN} \rightarrow \mathrm{N} \Delta)$ for off-shell kinematics. The model has no free parameters because the two-body input information is constrained by the experimental $\pi \mathrm{N}$ phase shifts. Predictions of the DKS model qualitatively agree with $p p \rightarrow p n \pi^{+}$spin observable data $^{6}$ whereas the agreement with $p p \rightarrow p p \pi^{0}$ data is marginal. ${ }^{7}$ One limitation of the model is that it contains only p-wave pion production. The importance of smaller amplitudes, such as the s-wave term, has been emphasized in analyses of pion absorption on $\mathrm{I}=0$ and $\mathrm{I}=1$ nucleon pairs in $\mathrm{s}$ - and $\mathrm{p}$-shell nuclei. ${ }^{8}$ The $n p \rightarrow p p \pi^{-}$reaction provides an excellent opportunity for detailed studies of the smaller pion-nucleon off-shell amplitudes.

\section{References}

[1] M. Kleinschmidt et al., Z. Physik A298, 253 (1980).

[2] W. Thomas et al., Phys. Rev. D 24, 1736 (1981).

[3] G. Mutchler, private communication.

[4] TRIUMF Proposal E372, N. E. Davison, spokesman, "Study of Single Pion Production in $n p \rightarrow p p \pi^{-} . "$

[5] W. M. Kloet and R. R. Silbar, Nucl. Phys. A338, 281 (1980); A426, 625 (1984); A338, 317 (1980); A364, 346 (1981).

[6] C. L Hollas et al., Phys. Rev. Lett. 55, 29 (1985).

[7] P. J. Riley et al., Phys. Lett. B 197, 23 (1987).

[8] M. A. Moinester et al., Phys. Rev. Lett. 52, 1203 (1984); H. Yokota et al., Phys. Rev. Lett. 57, 807 (1986); O. V. Maxwell and C. Y. Cheung, Nucl. Phys. A454, 606 (1986).

\section{Figure Captions}

Figure 1: Schematic diagram of the EXP1097 detector.

Figure 2: Top view of cylindrical drift chamber showing $\mathrm{HV}$ and sense wires.

Figure 3: Schematic diagram of the polarized target layout for Phase II. 

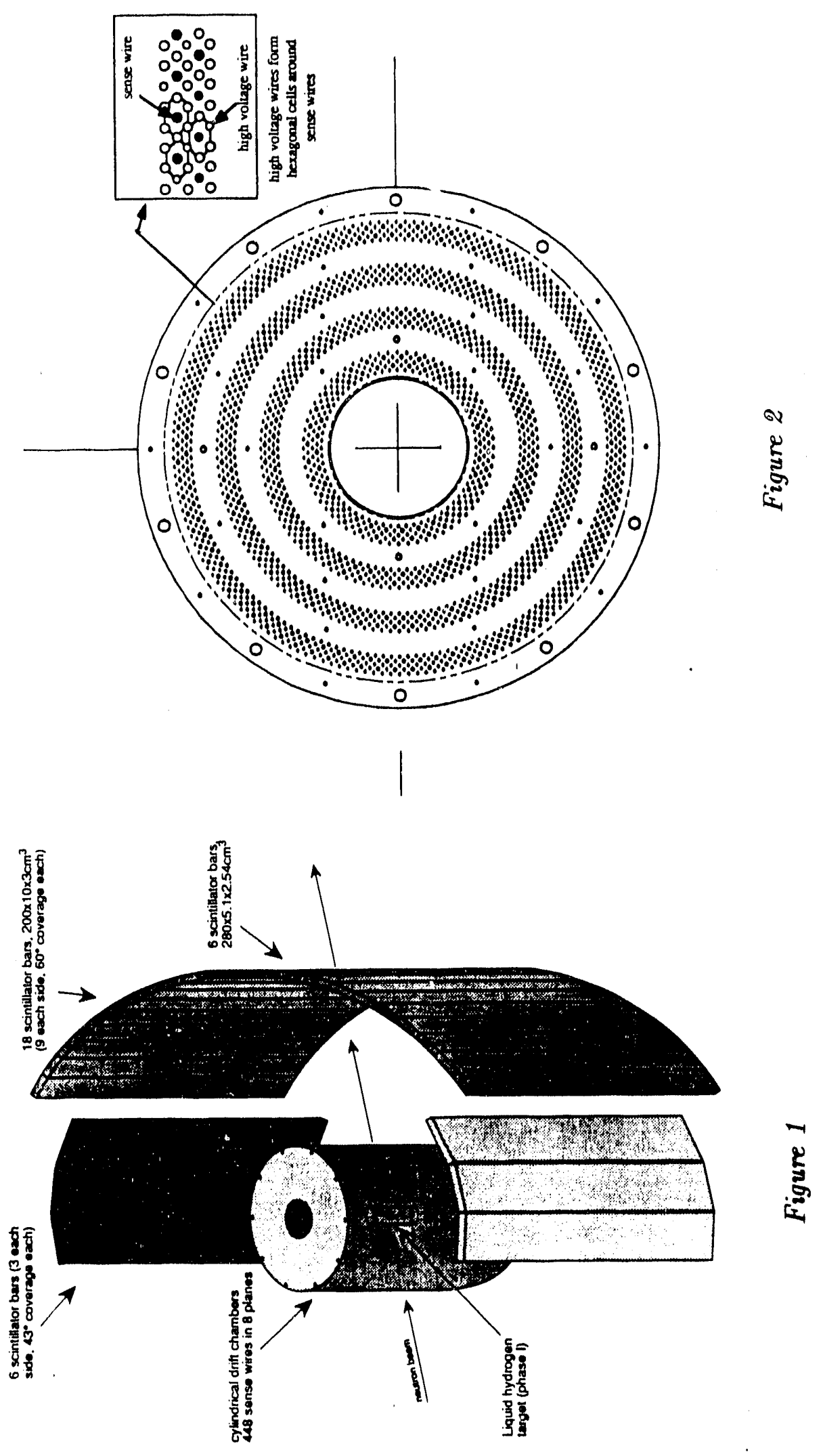
Figure 3

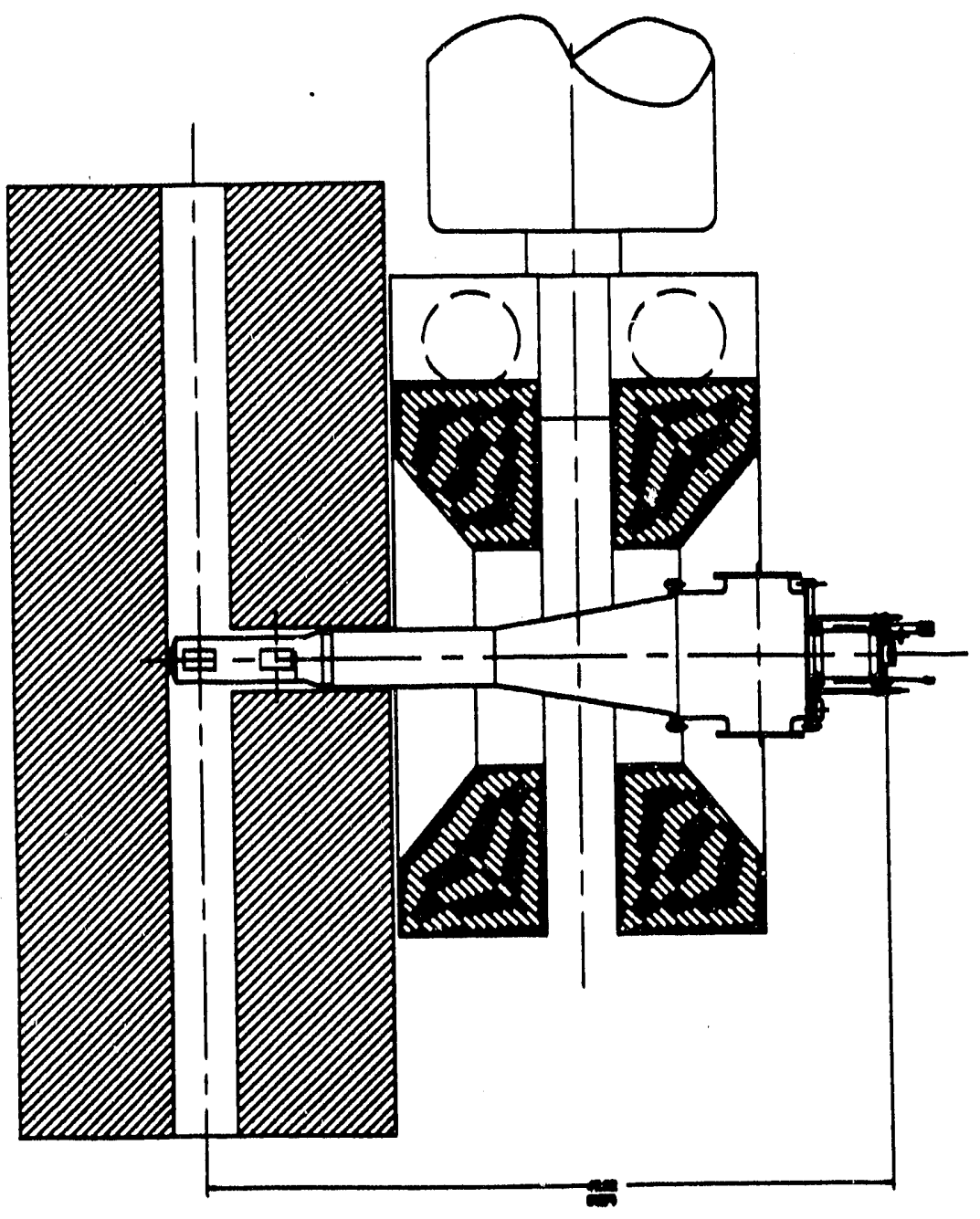




\subsection{EXP1145: Measurements of Complete Spin Observ- ables in the Quasifree Region for Medium Energy $\left(p, p^{\prime}\right)$}

(G. W. Hoffmann; Colorado; Rutgers; Georgia; Orsay; Minnesota;

LANL; X. Y. Chen, spokesperson)

Summary: The medium resolution spectrometer (MRS) at LAMPF will be used to measure a complete set of polarization transfer observables $\left(D_{i j}\right)$ and analyzing powers for ${ }^{40} \mathrm{Ca}\left(p, p^{\prime}\right)$ at $500 \mathrm{MeV}$ over the continuum region from 30 to $250 \mathrm{MeV}$ excitation energy at laboratory angles of $22^{\circ}$ and $29^{\circ}$. The data will cover the entire quasielastic region.

The study of spin-dependent nuclear response functions in the quasi-elastic continuum region is of great current interest owing to several predictions of important medium modifications which indicate shifts in the positions of the various quasifree responses and/or changes in their strengths. ${ }^{1}$ In this experiment the polarization transfer coefficients $D_{n n}, D_{\ell \ell}$ and $D_{s s}$ will be determined. These three quantities along with the unpolarized differential cross section allow four independent combinations of observables to be constructed. In plane wave Born approximation these four observable combinations can be directly related to specific spin-dependent nuclear response functions. These simple relations remain valid in the distorted wave limit to good accuracy. Two of these response functions correspond to the Coulomb and transverse spin responses measured in $\left(e, e^{\prime}\right) .^{2}$ The other two correspond to spin-longitudinal and spin-transverse (normal to the scattering plane) responses and can only be studied with hadronic probes. Theoretical models ${ }^{1,3}$ predict the longitudinal response to be lowered in energy, the ratio of the strengths of the longitudinal to transverse responses to be enhanced, etc.; theoretical calculations are also unable to quantitatively account for the Coulomb response in $\left(e, e^{\prime}\right) .^{2}$

The angles chosen for this experiment correspond to momentum transfers of 410 and $550 \mathrm{MeV} / \mathrm{c}$ which will allow direct comparison with the electron scattering data on ${ }^{40} \mathrm{Ca}^{2}$ The energy of $500 \mathrm{MeV}$ is chosen to minimize complications due to medium corrections to the effective nucleon-nucleon interaction, to avoid the strong absorption of the incident proton which occurs at higher energies $(800 \mathrm{MeV})$, and to allow operation in a region where the spin-dependent components of the nucleon-nucleon interaction are still significant relative to the spin-independent parts. Previous experiments ${ }^{3}$ obtained data at just the peak of the quasi-elastic region and were not at the same momentum transfer as the electron scattering data. It is hoped that these data will facilitate a consistent understanding of the spin-dependent nuclear response functions measured with electrons, protons and in $(p, n)$ charge exchange. 


\section{References}

[1] W. M. Alberico et al., Phys. Rev. C 38, 109 (1988).

[2] Z. E. Meziani et al., Phys. Rev. Lett. 52, 2130 (1984); J. Morgenstern, Nucl. Phys. A446, 315c (1985); M. Deady et al., Phys. Rev. C 33, 1897 (1986).

[3] T. A. Carey et al., Phys. Rev. Lett. 53, 144 (1984); L. M. Rees et al ., Phys. Rev. C 34, 627 (1986). 


\subsection{EXP1178: Polarization Asymmetry Measurements for the ${ }^{1} \overrightarrow{\mathbf{H}}\left(\pi^{-}, \pi^{0}\right) n$ Reaction Between 45 and $190 \mathrm{MeV}$}

(G. W. Hoffmann; ASU; NMSU; LANL; Minnesota; Colorado; Maryland;

TRIUMF; J. R. Comfort, G. Burleson, spokesmen)

Summary: Polarization asymmetry data will be taken at the LEP channel at LAMPF for the pion single charge-exchange (SCX) reaction ${ }^{1} \overrightarrow{\mathrm{H}}\left(\pi^{-}, \pi^{0}\right) n$ over the energy range from 45 to $190 \mathrm{MeV}$. No measurements of these fundamental $\pi \mathrm{N}$ quantities exist at energies below $190 \mathrm{MeV}$. Asymmetry measurements in combination with differential cross section data provide information about the relative phases between the $\pi \mathrm{N}$ spin-independent and spin-dependent amplitudes. The analysis of the low energy $(\sim 45 \mathrm{MeV})$ data will be useful for studying the nonresonant $\mathrm{P}_{11}$ phase shifts.

The pion-nucleon interaction is of fundamental importance in nuclear physics. It plays a dominant role in determining nucleon-nucleon scattering and is the lowest order ingredient needed in theoretical models of pion-nucleus scattering and reactions. In order to obtain the best possible experimental description of the $\pi \mathrm{N}$ system, including the spin-dependent amplitudes, one must measure as many observables as is feasible. Also, in order to study medium effects or exotic processes in pion-nucleus scattering, it is imperative that the elemental pion-nucleon amplitudes be accurately known.

The general form for the pion-nucleon scattering amplitude (including chargeexchange) is

$$
F_{\pi N}=f(\theta)+i g(\theta) \vec{\sigma} \cdot \hat{n},
$$

where $\hat{n}$ is normal to the scattering plane and $\vec{\sigma}$ is the Pauli spin operator for the nucleon. Both $f$ and $g$ can be decomposed into isoscalar and isovector terms. The SCX cross section measurements determine only the absolute magnitudes of the isovector parts of $f$ and $g$, whereas the polarization asymmetries are sensitive to the relative phases between the isovector parts of the two amplitudes.

Pion-nucleon phase shifts are usually determined from $\pi^{+}$and $\pi^{-}$elastic scattering data from hydrogen, including differential cross section and asymmetry measurements. Although there is a direct theoretical connection between the $\pi^{+} p$ and $\pi^{-} p$ amplitudes and the charge-exchange amplitudes, given by

$$
\sqrt{2} a^{0}=a^{+}-a^{-}
$$

the elastic data alone are insufficient to determine the observables for the chargeexchange process. The amplitudes $a^{0}, a^{+}$, and $a^{-}$correspond to charge-exchange and $\pi^{+}$and $\pi^{-}$elastic scattering from protons, respectively. The reason for the ambiguity is that experiments.cannot determine the relative phases between the 
$a^{+}$and $a^{-}$amplitudes. ${ }^{1} \mathrm{H}\left(\pi^{-}, \pi^{0}\right) n$ reaction data are still needed to provide an independent measure of the isovector amplitudes.

The lowest energy for which elementary pion SCX data exist is $192 \mathrm{MeV} .^{1}$ Recently, asymmetry measurements have been made at TRIUMF for $\pi^{+}$and $\pi^{-}$ elastic scattering from hydrogen between 98 and $263 \mathrm{MeV}{ }^{2}$ The measurements to be made here will either confirm the TRIUMF results or reveal deficiencies with existing data or phase shift solutions. The situation at lower energies is substantially worse since there are no measurements below $90 \mathrm{MeV}$ of pion elastic asymmetries. Furthermore, there are serious disagreements between several data sets and between data and phase shift analyses. ${ }^{3-5}$

The experiment will use a polarized hydrogen target, similar to that used for previous LAMPF experiments, ${ }^{6}$ and will use a ${ }^{3} \mathrm{He}$ evaporation refrigerator, a ${ }^{3} \mathrm{He}$ ${ }^{4} \mathrm{He}$ dilution refrigerator, and a room temperature electromagnet (Zoltan). The target material will be butanol, $\mathrm{C}_{4} \mathrm{H}_{9} \mathrm{OH}$, which is preferred over the previously used ethylene-glycol because it provides a higher ratio of hydrogen to contaminant (carbon and oxygen), namely 2:1 compared with 3:2. Hydrogen polarizations of about $80 \%$ have been achieved in tests with butanol in a ${ }^{3} \mathrm{He}$ refrigerator. The polarization should be $90 \%$ with a dilution refrigerator.

The experiment will be done in two stages. The first stage will be for energies above $100 \mathrm{MeV}$ using the full 2.5-T magnetic field for the polarized target magnet and $a{ }^{3} \mathrm{He}$ refrigerator. The second stage will cover the lower energies, will use a weaker magnetic field, and will use a dilution refrigerator. This staged approach is required by the kinematic conditions of the experiment. At energies above $100 \mathrm{MeV}$ the pion beam is bent from $50^{\circ}$ to $90^{\circ}$ by the $2.5 \mathrm{~T}$ field of Zoltan. At lower energies the beam would be bent much more and would interfere with other apparatus such as the target support structure and so would not be able to be monitored effectively. Thus, a smaller magnetic field is required at the lower energies and in order to maintain sufficient polarization the target material temperature must be reduced. This requires the dilution refrigerator. Fields as low as $1 \mathrm{~T}$ can be handled in this way. The ${ }^{3} \mathrm{He}$ refrigerator is preferred at the higher energies because it is easier to use and more reliable.

The $\pi^{0}$ spectrometer will be placed at fixed angles and the polarization direction of the target will be reversed periodically. The large angular acceptance of the $\pi^{0}$ spectrometer will permit several angles to be measured simultaneously.

\section{References}

[1] G. Kim, Ph. D. thesis, UCLA, LANL Report LA-11273-T (1988).

[2] M. E. Sevior et al., 1988 Progress Report of the Nuclear Physics Laboratory of the Univ. of Colorado.

[3] P. B. Siegel and W. R. Gibbs, Phys. Rev. C 33, 1407 (1986). 
[4] M. E. Sadler, in Physics with Light Mesons, The Second Intern. Workshop on $\pi \mathrm{N}$ Physics, Los Alamos National Laboratory, LANL Report LA-11184-C (1987), p. 200.

[5] A. Bagheri et al., Phys. Rev. C 38, 885 (1988).

[6] See LAMPF proposals for EXPS 955, 1023, and 1025. See also J. J. Jarmer, in the proceedings of the LAMPF Workshop on Physics with Polarized Nuclear Targets, Los Alamos National Laboratory, LAMPF Conf. Report LA-10772C (1986), p. 1. 


\title{
3.5 EXP1205: Precision Measurement of $D_{N N}$ for ${ }^{13} \mathrm{C}(\vec{p}, \vec{p})$ at $500 \mathrm{MeV}$
}

\author{
(G. Hoffmann, L. Ray, D. Read, S. Worm; LANL; Rutgers; Georgia; \\ G. W. Hoffmann, J. F. Amann, C. Glashausser, spokesmen)
}

Summary: The high resolution spectrometer (HRS) and its focal plane polarimeter (FPP) will be used to make precision measurements of the polarization transfer quantity $D_{N N}$ for $500 \mathrm{MeV}$ polarized proton elastic scattering from ${ }^{13} \mathrm{C}$. The analysis of these data will provide information about specific components of the NN effective interaction and will impose important, new constraints on relativistic and nonrelativistic scattering models. This information complements that obtained from the elastic $\vec{p}+\hat{n}$-type polarized ${ }^{13} \mathrm{C}$ data and from $\left(\vec{p}, \vec{p}^{\prime}\right)$ data. Measurements will be made in the angular region of the first diffractive minima of the differential cross section (around $16^{\circ}$ laboratory angle). Using $a^{13} \mathrm{C}-{ }^{12} \mathrm{C}$ mixed target, data for $p+{ }^{12} \mathrm{C}$ will be obtained simultaneously with the ${ }^{13} \mathrm{C}$ data in order to empirically eliminate systematic errors. Statistical accuracy of \pm 0.01 will be obtained.

The principal goals of intermediate energy proton-nucleus scattering experiments are to provide data which facilitate the study of the effective two-body interaction in nuclei, the determination of nuclear structure, the study of reaction mechanisms at medium energies, and the testing of relativistic and nonrelativistic scattering theories. ${ }^{1-4}$ The largest effort in this field has involved proton elastic and inelastic scattering from even-even target nuclei; the first few natural parity collective excitations have been studied. Analyses of these data have been aimed at studying the dominant, isoscalar non-spin-transfer components of the NN force, the isoscalar ground state matter densities and collective transition densities, and comparing relativistic and nonrelativistic scattering models. Both the relativistic and nonrelativistic models use fundamental NN interactions and realistic nuclear structure information. Quantitative, parameter free descriptions of proton-(spinzero)nucleus elastic scattering and inelastic excitation of natural parity, collective states, using either the relativistic or nonrelativistic models, are gradually becoming a reality.

Much less work has been done for reactions involving spin and/or isospin transfer. These reactions are sensitive to the spin-transfer and isovector components of the NN force and to single particle aspects of nuclear structure. At intermediate energies studies of these topics include $\left(\vec{p}, \vec{p}^{\prime}\right)$ for non-natural parity transitions, $(\mathrm{p}, \mathrm{n})$ charge-exchange $500 \mathrm{MeV} \vec{p}+{ }^{13} \overrightarrow{\mathrm{C}}$ elastic scattering analyzing power $A_{\text {ooon }}$ and spin correlation parameter $A_{o o n n},{ }^{5}$ and $D_{N N}$ at $500 \mathrm{MeV}$ for ${ }^{13} \mathrm{C}(\vec{p}, \vec{p})$ elastic scattering $^{6}$ (see Fig. 1). Relativistic and NR models describe the inelastic data with varying success, with no-clear preference for either one. ${ }^{7}$ Neither approach 
is able to describe the charge-exchange data, ${ }^{8}$ while both approaches provide reasonable, qualitative descriptions of the polarized ${ }^{13} \mathrm{C}$ elastic scattering data. ${ }^{5}$ The existing elastic $D_{N N}$ data (see Fig. 1) for ${ }^{13} \mathrm{C}$ are of insufficient quality to test reaction models or effective interactions. In general, our present understanding of spin and isospin transitions at intermediate energies is unsatisfactory and more work in this area is necessary.

A precision measurement of $D_{N N}$ for proton elastic scattering from ${ }^{13} \mathrm{C}$ will allow important work to proceed in these studies. Specifically, such data will enable new tests of relativistic and nonrelativistic models and will provide effective interaction information that complements that obtained from the elastic $\vec{p}+\hat{n}$ type polarized ${ }^{13} \mathrm{C}$ data and from $\left(\vec{p}, \vec{p}^{\prime}\right)$ data. The presently available $D_{N N}$ elastic scattering data from odd nuclei include only 72 and $500 \mathrm{MeV}{ }^{13} \mathrm{C}(\vec{p}, \vec{p})$ data and $65 \mathrm{MeV}^{15} \mathrm{~N}(\vec{p}, \vec{p})$ data. The low energy data ${ }^{9,10}$ are not suitable for studies of the relativistic versus nonrelativistic approaches. The existing $500 \mathrm{MeV}$ data are not of sufficient quality for studying the effective interaction or relativistic versus NR issues.

The quantity $D_{N N}$ for elastic scattering from even-even $\left(J^{\pi}=0^{+}\right)$targets is unity, corresponding to spin-flip probability zero. For ${ }^{13} \mathrm{C}$ with ground state $J^{\pi}=\frac{1}{2}^{-}$both $\Delta J=0,1$ contributions are allowed. $\Delta J=1$ permits $D_{N N}$ to differ from unity. The $500 \mathrm{MeV} \vec{p}+{ }^{13} \overrightarrow{\mathrm{C}}$ spin-flip probability was predicted using the RIA-DWBA model of Ref. 4 and a NRIA-DWBA model. For the relativistic model the form taken for the Lorentz invariant $\mathrm{NN}$ effective interaction is the local form,

$$
F=F_{S}+F_{P} \gamma_{1}^{5} \gamma_{2}^{5}+F_{V} \gamma_{1}^{\mu} \gamma_{2 \mu}+F_{A} \gamma_{1}^{5} \gamma_{1}^{\mu} \gamma_{2}^{5} \gamma_{2 \mu}+F_{T} \sigma_{1}^{\mu \nu} \sigma_{2 \mu \nu}
$$

The proton-nucleus scattering amplitude in the DWBA approximation is given by ${ }^{4}$

$$
f_{m_{s}^{\prime} \mu^{\prime} ; m_{s} \mu}\left(\vec{k}, \vec{k}^{\prime}\right)=f_{m_{s}^{\prime} m_{\ominus}}^{c o r e}\left(\vec{k}, \vec{k}^{\prime}\right) \delta_{\mu^{\prime} \mu}-\frac{m}{2 \pi(\hbar c)^{2}}\left\langle\chi_{c, \vec{k}^{\prime} m_{s}^{\prime}}^{(-)} u_{n \ell j \mu^{\prime}}|F| u_{n \ell j \mu} \chi_{c, \vec{k} m_{\ominus}}^{(+)}\right\rangle,
$$

where $f^{\text {core }}$ is the elastic scattering amplitude from the $\left({ }^{12} \mathrm{C}\right)$ core, $u_{n \ell j \mu}$ is the relativistic bound state wave function ( $1 p_{\frac{1}{2}}$, neutron), and $\chi_{c, \vec{k} m}$, is the relativistic, 4-component distorted wave function for the core nucleons corresponding to $f$ core . The bound state wave function, $u_{n \ell j \mu}(\vec{r})$, contains upper and lower components, $\phi$ and $\lambda$, respectively, and for the purpose of studying sensitivities was assumed to be a simple $1 p_{\frac{1}{2}}$ eigenstate of the relativistic mean field ${ }^{11}$ (RMF) potential for ${ }^{12} \mathrm{C}$. For some of the calculations discussed here the nonrelativistic limit of $\lambda$ was used. This limit was obtained by solving the single particle bound state Dirac equation in the limit of zero scalar and vector binding potentials. Pseudovector (PV) rather than pseudoscalar (PS) forms for the Lorentz invariant $\mathrm{NN}$ interaction were also used. $^{4}$

The five invariant amplitudes in Eq. (3.3) were obtained from the Pauli representation of the NN scattering amplitudes by the transformation described in Ref. 3, using Arndt's SP82 phase shift solution. The relativistic distorted waves and core scattering amplitudes were generated from either the RIA - Dirac equation 
model ${ }^{3}$ for $p+{ }^{12} \mathrm{C}$ or the Dirac phenomenological optical model fit to the $p+{ }^{12} \mathrm{C}$ $500 \mathrm{MeV}$ elastic scattering data (see Ref. 6).

For the nonrelativistic predictions the DWBA model of Ref. 4 was used. The same NN phase shifts were used as in the relativistic calculations. The $1 p_{\frac{1}{2}}$ valence neutron wave function was taken to be the same as the upper component in the relativistic valence nucleon wave function. The core amplitudes and distorted waves were obtained from the Schrödinger equivalent optical potential of the Dirac phenomenological fit to the $\vec{p}+{ }^{12} \mathrm{C}$ elastic scattering data by solving the Schrödinger equation with relativistic kinematics.

In Fig. 1 RIA-DWBA predictions are shown with the existing $D_{N N}$ data for $\vec{p}+{ }^{13} \mathrm{C}$ at $500 \mathrm{MeV}$. The curves are discussed in detail in Ref. 6. Briefly the solid and dashed lines resulted from using PV and PS forms for the NN invariant amplitudes, respectively. The curves in the upper (lower) half of the figure resulted from using the RIA (Dirac phenomenological) optical potential to generate the relativistic distorted waves and core scattering amplitudes. In Fig. 2 the sensitivity of the RIA-DWBA model predictions for $D_{N N}$ to the lower component of the $1 p_{\frac{1}{2}}$ bound state wave function is displayed. The solid and dashed curves were generated by using the RMF and NR limit for the lower component, respectively. Both calculations assumed the pseudoscalar form for the $\mathrm{NN}$ invariant amplitudes and the RIA model for the distorted waves and core amplitudes. In Fig. 3 nonrelativistic and relativistic predictions are compared. Dirac phenomenological optical potentials were used to generate the distorted waves and core scattering amplitudes. The shaded band encompasses the relativistic predictions while the dashed curve indicates the NR result.

In the language of the Lorentz invariant form of the NN interaction, the spinflip probability for this case is determined primarily by the PS invariant and to a lesser extent by the space-like tensor component. It is particularly sensitive to the choice of PS or PV forms (if $\lambda^{R M F}$ is assumed and not $\lambda^{N R}$ ). In contrast, for elastic scattering from polarized targets, the PS component is not important for the $\hat{n}$-type polarized ${ }^{13} \mathrm{C}$ observables; ${ }^{4}$ its study requires the much more difficult experiment involving an $\hat{s}$-type polarized target. ${ }^{4}$ It is also sensitive to the lower component strength of the valence wave function (for the PS form) (Fig. 2). There are also significant differences predicted between the NR model and the centroid of the range of the relativistic model predictions as shown in Fig. 3.

The predicted spin-flip strength is affected somewhat by the aissumptions for the core scattering amplitude. This quantity can, however, be tightly constrained in the analysis, since precision unpolarized $\vec{p}+{ }^{13} \mathrm{C}$ data have been obtained. The dependences on the distorted wave model and the large, upper component of the valence wave function are weak. Multistep contributions in the region of the first minimum can be evaluated with the recently completed Dirac coupled-channels code $^{12}$ which includes the full proton + odd-nucleus relativistic optical potential.

The principal advantages of this method of studying the relativistic versus nonrelativistic question and determining the spin-transfer components of the NN 
effective interaction are: (1) The nuclear structure uncertainties are minimized for elastic scattering relative to $\left(p, p^{\prime}\right)$; the polarized ${ }^{13} \mathrm{C}$ data can be used to help "fine tune" the valence wave function. (2) These measurements address much of the same physics (i.e., PS versus PV forms, strength of the pseudoscalar invariant, lower component enhancement, etc.) as in the $\hat{s}$-type polarized ${ }^{13} \mathrm{C}$ elastic scattering experiment but are obviously much easier to do.

It is crucial to the experiment to have well-resolved elastic peaks for ${ }^{12} \mathrm{C}$ and ${ }^{13} \mathrm{C}$ for accurate determination of the $D_{N N}$. Near a laboratory scattering angle of $16^{\circ}$ (corresponding to center-of-momentum scattering angles near $18^{\circ}$ for 500 $\mathrm{MeV} p+{ }^{12,13} \mathrm{C}$ ), the elastically scattered protons from $500 \mathrm{MeV} p+{ }^{12,13} \mathrm{C}$ differ in energy by $300 \mathrm{keV}$. An intrinsic resolution of $80 \mathrm{keV}$ for the HRS should be easy at $500 \mathrm{MeV}$ using a $50 \mathrm{mg} / \mathrm{cm}^{2}$ (combined ${ }^{13} \mathrm{C}-{ }^{12} \mathrm{C}$ ) target. Simultaneous $p+{ }^{13} \mathrm{C}$ and $p+{ }^{12} \mathrm{C}$ data acquisition will allow systematic errors to be removed in the experiment, since $D_{N N}=1$ for ${ }^{12} \mathrm{C}$. Sufficient running time will assure good statistics $( \pm 0.01)$.

A precision measurement of $D_{N N}$ in the region of the first diffractive minimum will at least constrain and may eliminate some of the relativistic models. They will also critically test the NR models.

\section{References}

[1] J. A. McNeil, J. Shepard, and S. J. Wallace, Phys. Rev. Lett. 50, 1439, 1443 (1983).

[2] B. C. Clark et al., Phys. Rev. Lett. 50, 1644 (1983).

[3] L. Ray and G. W. Hoffmann, Phys. Rev. C31, 538 (1985).

[4] L. Ray et al., Phys. Rev. C37, 1169 (1988).

[5] G. W. Hoffmann et al., Phys. Rev. Lett. 65, 3096 (1990).

[6] G. W. Hoffmann et al., Phys. Rev. C 41, 1651 (1990).

[7] E. Rost and J. R. Shepard, Phys. Rev. C35, 681 (1987); K. H. Hicks et al., Phys. Lett. 201B, 29 (1988); J. Piekarewicz, Phys. Rev. C32, 1693 (1985).

[8] D. Ciskowski et al., Bull. Am. Phys. Soc. 33, 1583 (1988); D. Ciskowksi, $\mathrm{Ph}$. D. thesis, The University of Texas at Austin, 1989; T. N. Taddeucci, private communication.

[9] B. v. Przewoski et al., Phys. Rev. Lett. 64, 368 (1990).

[10] T. Nakano et al., Phys. Lett. B 240, 301 (1990).

[11] C. J. Horowitz and B. B. Serot, Nucl. Phys. A368, 503 (1981). 
[12] R. L. Mercer and B. C. Clark, private communication.

\section{Figure Captions}

Figure 1: Data and RIA-DWBA predictions for $D_{N N}$ for $500 \mathrm{MeV} \vec{p}+{ }^{13} \mathrm{C}$ elastic scattering from Ref. 6. The curves are discussed in the text.

Figure 2: Predicted sensitivity in $D_{N N}$ to $\lambda(r)$ in the RIA-DWBA model (from Ref. 4). The curves are discussed in the text.

Figure 3: Relativistic and nonrelativistic DWBA predictions for $500 \mathrm{MeV}$ ${ }^{13} \mathrm{C}(\vec{p}, \vec{p}) D_{N N}$. The shaded band indicates the range of predictions of the relativistic DWBA model. The upper (lower) limit of the shaded band corresponds to the PV (PS) form for the NN invariant amplitude. The NR prediction is indicated by the dashed curve.

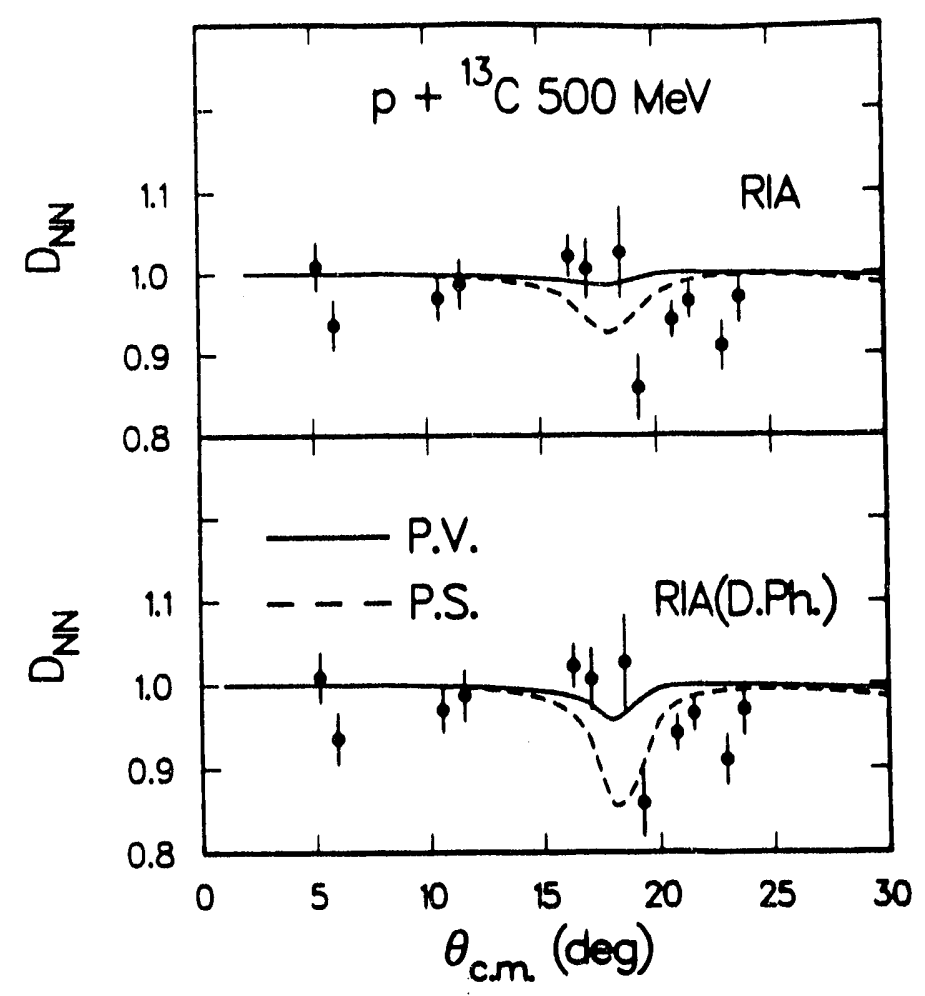

Figure 1 


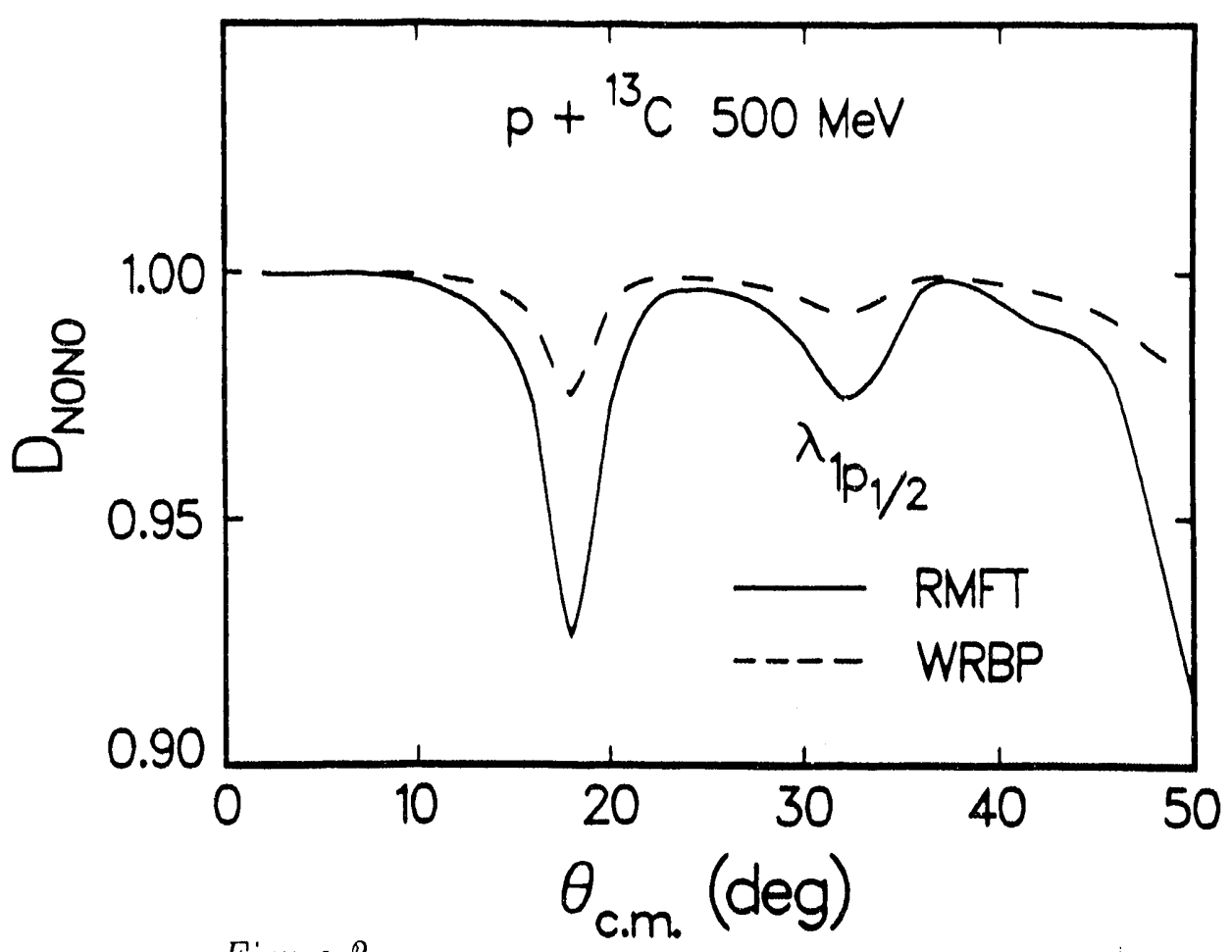

Figure 2

$p+13 C 500 \mathrm{MeV} ;$ RIA vs NRIA

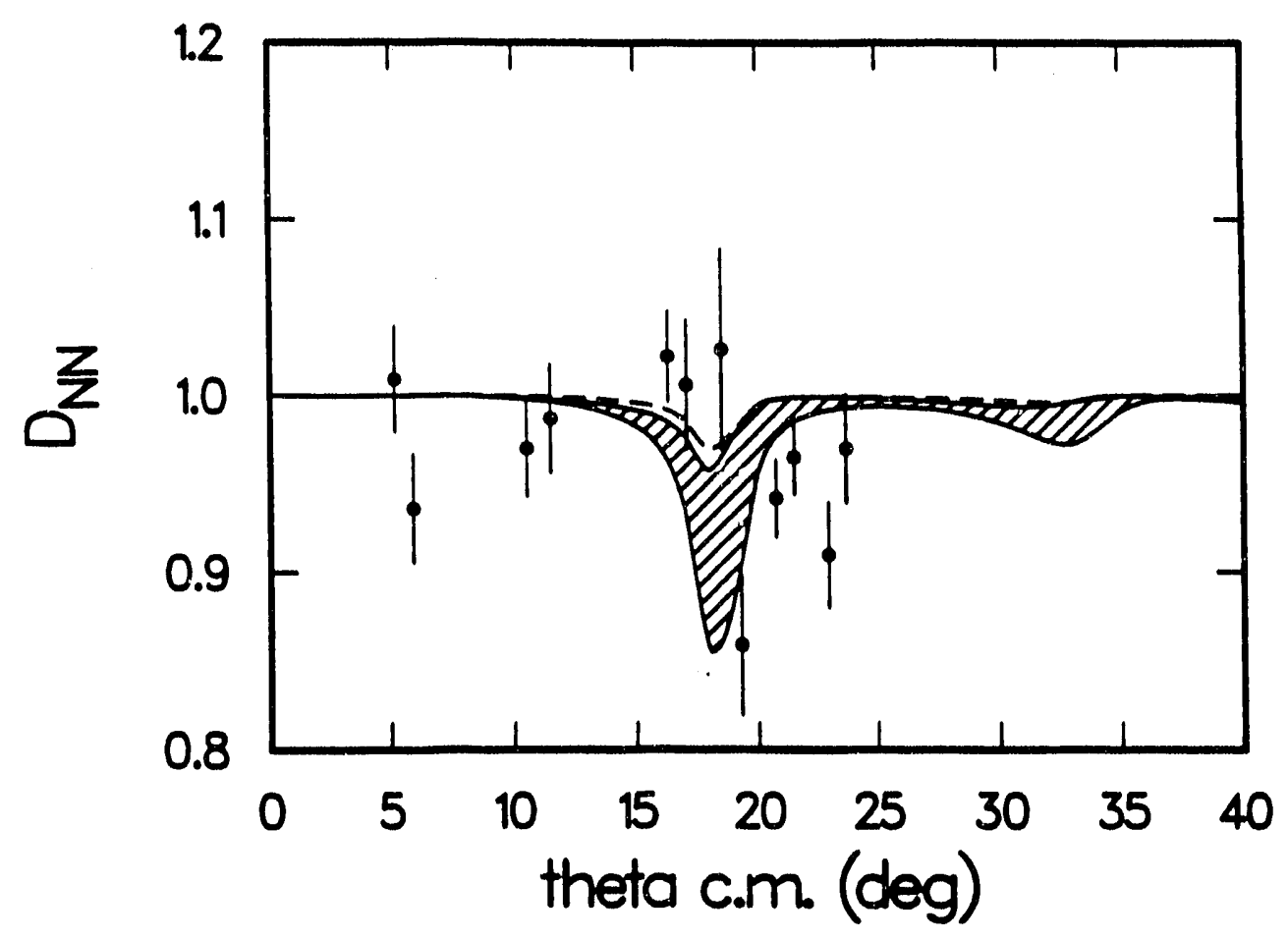

Figure. $3 . .$. 


\title{
3.6 EXP1206: Polarized Proton Elastic Scattering from Polarized ${ }^{6} \mathrm{Li}$ at $500 \mathrm{MeV}$
}

\author{
(G. W. Hoffmann, L. Ray, D. Read, S. Worm; LANL; NMSU; ANL; \\ ASU; Serpukhov; Kyoto; Old Dominion; TAMU; Maryland; Minnesota; \\ Udine; G. W. Hoffmann, G. R. Burleson, K. Koch, spokesmen)
}

Summary: The high resolution spectrometer (HRS) and a polarized ${ }^{6} \mathrm{Li}$ target will be used to take target spin observable data for $500 \mathrm{MeV}$ polarized proton elastic scattering. Differential cross section, analyzing power, and spin-depolarizationrotation data will be taken with unpolarized targets. The purpose of these measurements is: (1) to introduce a new degree of freedom into the medium energy scattering problem, namely the spin of the target nucleus; (2) to search for new physics; (3) to study the spin-transfer components of the isoscalar part of the nucleon-nucleon effective interaction in the nuclear medium; (4) to study nuclear structure; and (5) to perform a significantly cleaner measurement of proton elastic scattering from a polarized nuclear target than was possible in the first such experiment, LAMPF EXP955, $\vec{p}+{ }^{13} \overrightarrow{\mathrm{C}}$. For the polarized target runs, the target vector analyzing power, $A_{\text {ooon }}$, and spin correlation vector analyzing power, $A_{\text {oonn }}$, will be obtained. We hope to obtain statistical accuracies of $\pm(1-2) \%$ for $d \sigma / d \Omega, \pm(0.01-0.02)$ for $A_{y}, \pm(0.02-0.04)$ for the $D_{i j}$, and $\pm(0.01-0.03)$ for $A_{\text {ooon }}, A_{\text {oonn }}$.

The interest in polarized nuclear target physics at LAMPF began in 1986 with the LAMPF workshop, Physics with Polarized Nuclear Targets. ${ }^{1}$ It served as a catalyst for a series of proposals for proton and pion scattering from polarized ${ }^{13} \mathrm{C}$. The first was EXP955, polarized proton elastic scattering from polarized ${ }^{13} \mathrm{C}$ at $500 \mathrm{MeV}$ (Ref. 2). The other three experiments, EXP1023, EXP1025, and EXP1172 involve pion single charge-exchange from ${ }^{13} \overrightarrow{\mathrm{C}}$, pion elastic/inelastic scattering from ${ }^{13} \overrightarrow{\mathrm{C}}$, and $\left(\pi, \pi^{\prime} p\right)$ from polarized ${ }^{7} \mathrm{Li}$. The first round of polarized target experiments is nearing completion. Analysis and interpretation of the data will continue for some time as refinements in the data analysis and improvements in the reaction models continue. It is now time to move on to the next series of polarized target measurements.

In designing the next proton scattering experiment we must build on the experience of EXP955. In the ${ }^{13} \mathrm{C}$ experiment, obtaining the polarized target observables required that very accurate differences in yields of the order of $1 \%$ be measured. This was complicated by the fact that the ${ }^{16} \mathrm{O}$ elastic peak (from the ${ }^{16} \mathrm{O}$ in the ethylene glycol target material) was unresolved from the ${ }^{13} \mathrm{C}$ elastic peak and was of :omparable magnitude. Also the inhomogeneity of the target material (frozen beads) resulted in small, uncontrollable changes in effective target thickness from run-to-run. A very difficult and delicate procedure was developed in order to ac- 
count for the change in effective target thickness between the target "up" and target "down" runs. ${ }^{2}$ A new experiment should not suffer from these problems.

This experiment offers several improvements over EXP955. (1) The ${ }^{6} \mathrm{Li}$ and the much smaller (but polarized) ${ }^{7} \mathrm{Li}$ contaminant will be resolved over most of the angular range. We will use an isotopically enriched $(95 \%)^{6} \mathrm{LiH}$ target. A separate measurement with a polarized ${ }^{7} \mathrm{Li}$ target will be made in order to allow subtraction of the small, but significant ${ }^{7} \mathrm{Li}$ background. (2) With both the HRS and a target polarimeter, $\vec{p}+{ }^{1} \overrightarrow{\mathrm{H}}$ elastic data will be carefully monitored in order to allow accurate determination of target and beam polarization; this will also permit accurate determination of run to run relative target thickness.

Our motivation for further polarized target experiments has shifted somewhat from that in the original ${ }^{13} \overrightarrow{\mathrm{C}}$ experiment. We still contend that only by conducting accurate, high quality measurements of entirely new observables is there any chance of finding interesting, new physics. Measurements involving the spin degree of freedom of the target nucleus certainly provide such an opportunity. We also continue to believe that these data can be used to study the elusive spin-transfer components of the effective nucleon-nucleon (NN) interaction in the nuclear medium. We argue that studies of these parts of the nuclear force in the elastic channel, as compared to studies involving inelastic excitations, offer the advantage of reduced uncertainty in the nuclear structure (only the ground state wave function is required) and reduced uncertainty in the reaction mechanism (e.g. multistep processes are less likely to be important). Measurements of elastic channel observables with protons provide nuclear structure information complementary to that obtained with electromagnetic probes. We hope that analyses of such data may eventually be able to shed light on the question of relativistic enhancement of the lower component of the nucleon bound state wave functions in nuclei, but we do not place strong emphasis on whis. Also tests of relativistic versus nonrelativistic descriptions of just that part of the scattering amplitude involving the valence nucleons is not emphasized. We wish to focus attention on the new degrees of freedom associated with target spin, the studies of new parts of the NN effective interaction, and the advantages offered by elastic scattering.

The relativistic and nonrelativistic DWBA reaction models developed for $p+{ }^{13} \mathrm{C}$ (Ref. 3) have been modified to describe scattering from a spin 1 target. For the relativistic model, the usual local form $^{3}$ for the Lorentz invariant $\mathrm{NN}$ effective interaction was used. We assume that the ${ }^{6} \mathrm{Li}$ ground state consists of a proton and neutron in the $1 p_{\frac{3}{2}}$ orbital coupled to $J^{\pi}, T=1^{+}, 0$ and lying outside a closed, inert ${ }^{4} \mathrm{He}$ core. 'The wave function is therefore

$$
\begin{aligned}
\mid{ }^{6} \mathrm{Li}, J=1, M ; T= & 0\rangle=\frac{1}{\sqrt{2}} \sum_{m, t}\left(\frac{3}{2} m \frac{3}{2}, M-m \mid 1 M\right)\left(\frac{1}{2} t \frac{1}{2}-t \mid 00\right) \\
& \times a_{1 p_{\frac{3}{2}}, M-m,-t}^{\dagger} a_{1 p_{\frac{3}{2}}, m, t}^{\dagger}|C\rangle,
\end{aligned}
$$

where $a^{\dagger}$ is a particle creation operator, and $|C\rangle$ represents the ${ }^{4} \mathrm{He}$ core. The $p+{ }^{6} \mathrm{Li}$ first order optical potential was evaluated using the invariant amplitudes 
and this ground state wave function. The $p+{ }^{6} \mathrm{Li}$ elastic scattering amplitude in the DWBA approximation is

$$
\begin{aligned}
& f_{m_{s}^{\prime} M^{\prime} ; m_{s} M}\left(\vec{k}, \vec{k}^{\prime}\right)=f_{m_{s}^{\prime} m_{s}}^{\text {core }}\left(\vec{k}, \vec{k}^{\prime}\right) \delta_{M^{\prime} M} \\
+ & 2 \sum_{m}\left(\frac{3}{2} m \frac{3}{2}, M-m \mid 1 M\right)\left(\frac{3}{2} m \frac{3}{2}, M^{\prime}-m \mid 1 M^{\prime}\right) f_{m_{s}^{\prime}, M^{\prime}-m_{i} m_{s}, M-m}^{s p_{1}^{\prime} \text { soscalar }}
\end{aligned}
$$

where $M, M^{\prime}= \pm 1,0$ and $f^{s p_{1} \text { Isoscalar }}$ is the amplitude for a $p_{\frac{3}{2}} \rightarrow p_{\frac{3}{2}}$ transition. This is given by ${ }^{3}$

$$
f_{m_{s}^{\prime} \mu^{\prime} ; m_{s} \mu}^{s p, I s c a l a r}\left(\vec{k}, \vec{k}^{\prime}\right)=-\frac{m}{2 \pi(\hbar c)^{2}}\left\langle\chi_{c, \vec{k}^{\prime} m_{s}^{\prime}}^{(-)} u_{n \ell j \mu^{\prime}}\left|F^{I s o s c a l a r}\right| u_{n \ell j \mu} \chi_{c, \vec{k} m_{s}}^{(+)}\right\rangle,
$$

where only the isoscalar portion of the $\mathrm{NN}$ invariant amplitudes contribute. In this equation $f^{\text {core }}$ is the elastic scattering amplitude due to the $\left({ }^{4} \mathrm{He}\right)$ core plus the scalar and time-like vector $(\Delta J=0)$ parts of the valence nucleon contribution to the optical potential. The relativistic bound state wave function $\left(1 p_{\frac{3}{2}}\right)$ is denoted by $u_{n \ell j \mu}$, and $\chi_{c, \vec{k} m_{s}}$ is the relativistic, 4-component distorted wave function corresponding to $f^{\text {core }}$.

$\phi$ and $\lambda$ are the upper and lower components of the bound state wave function, $u_{n \ell j \mu}$, respectively. The upper component is assumed to be the $1 p_{\frac{3}{2}}$ eigenstate of a nonrelativistic binding potential. The lower component $\lambda$ was obtained by solving the single-particle bound state Dirac equation with realistic scalar and vector binding potentials. For some of the calculations discussed in the following, the nonrelativistic limit of $\lambda$ was used. The nuclear structure model assumed here is admittedly crude. However shell model wave functions ${ }^{4}$ and electron scattering data $^{5,6}$ are available for use in future analyses of data.

The Lorentz invariant amplitudes were obtained from the Pauli representation of the NN scattering amplitudes by the transformation described in Ref. 7, using just the isoscalar parts of Arndt's SP82 phase shift solution. The relativistic distorted waves and core scattering amplitudes were generated from the RIA - Dirac equation model ${ }^{7}$ for $p+{ }^{6} \mathrm{Li}$ (scalar and time-like vector parts of opical potential only) using electron scattering information to fix the target density.

For the nonrelativistic predictions the same DWBA model described here, and in Ref. 3, was used. The same NN phase shifts were used as in the relativistic calculations. The nonrelativistic $1 p_{\frac{3}{2}}$ valence neutron wave function was used. The core amplitudes and distorted waves were obtained from the Schrödinger equivalent optical potential of the RIA-Dirac equation model for $p+{ }^{6} \mathrm{Li}$ by solving the Schrödinger equation with relativistic kinematics.

It is important that the unpolarized $p+{ }^{6} \mathrm{Li}$ elastic observables be obtained so that realistic core scattering amplitudes and distorted wave functions are available for analysis of the polarized target data. (This was done for ${ }^{13} \mathrm{C}$ in EXP955, see Ref. 8.) For $\hat{n}$-type polarized ${ }^{6} \mathrm{Li}$ targets and $\hat{n}$-type polarized beams, the relevant observables are $A_{o o \text { ön }} ;-A_{o o n n}, A_{o o o, n n}$, and $A_{o o n, n n}$ (see Ref. 9 for notation 
for observables), where $n(n n)$ denotes a vector (tensor) polarization. Finally, the same reaction model has also been applied to $\vec{p}+{ }^{7} \overrightarrow{\mathrm{Li}}$.

Fig. 1 shows several RIA-DWBA results for $500 \mathrm{MeV} p+{ }^{6} \mathrm{Li}$ elastic scattering observables: the differential cross section $d \sigma / d \Omega$, analyzing power $\left(A_{y}\right)$, spin rotation $D_{\text {solo }}$, target vector polarization observables $A_{\text {ooon }}$ and $A_{\text {oonn }}$, target tensor polarization observables $A_{o o o, n n}$ and $A_{o o n, n n}$, and polarization transfer $K_{n o o n}$. The directions are defined in the c.m. in terms of the initial and final c.m. momenta $\vec{k}$ and $\vec{k}^{\prime}$, respectively, using $\hat{n}=\left(\vec{k} \times \vec{k}^{\prime}\right) /\left|\vec{k} \times \vec{k}^{\prime}\right|, \hat{s}=\left(\vec{k}^{\prime}-\vec{k}\right) /\left|\vec{k}^{\prime}-\vec{k}\right|$, and $\hat{\ell}=\left(\vec{k}^{\prime}+\vec{k}\right) /\left|\vec{k}^{\prime}+\vec{k}\right|$. The solid curves correspond to results using a relativistic $\lambda$ and the pseudoscalar (PS) form for the NN amplitudes. The broken curves correspond to $\lambda^{N R}$ and the PS form. For $A_{\text {ooon }}$ and $A_{\text {oonn }}$ the relativistic enhancement of $\lambda$ causes a $\sim 15 \%$ increase in the magnitudes of the observables. There is ro sensitivity to PS versus PV since the pseudoscalar portion of the invariant amplitude is mainly isovector. The tensor observables are quite small, being much less than 0.1 while the polarization transfer quantity, $K_{\text {noon }}$, is very sinular to $A_{o o n n}$. Fig. 2 compares RIA-DWBA and NRIA-DWBA results for the polarized target observables. The unpolarized target observables are virtually identical for the two cases. The NRIA-DWBA predictions for $A_{\text {ooon }}$ and $A_{\text {oonn }}$ are about $20 \%$ smaller than the RIA-DWBA predictions and very similar in shape. These results show the expected order-of-magnitude of the polarized target observables, which turn out to be fairly large for this type of quantity. The calculations indicate little sensitivity to relativistic effects for both the bound and scattered nucleon.

In Fig. 3 we show the separate contributions to $A_{\text {ooon }}$ and $A_{\text {oonn }}$ of each part of the NN invariant amplitude. Each of the calculations assumes a relativistic lower component and the PS form. The solid curves correspond to using the full NN invariant amplitude and are the same as the solid curves in Fig. 1. Calculations using just the isoscalar parts of $F_{P}, F_{V}, F_{A}$, and $F_{T}$ for the valence nucleon scattering amplitude resulted in the dashed, dotted, long-dashed-dotted, and short-dasheddotted curves, respectively. From these figures we see that the space-like vector amplitude is dominant, as in the $p+{ }^{13} \mathrm{C}$ case,${ }^{3}$ with significant cancellations occurring between the axial vector and tensor contributions. Thus our main interest is in studying the isoscalar part of the space-like vector interaction and the associated valence nucleon wave function.

The experiment naturally divides itself into 3 phases: (1) cross section and analyzing power measurements using polarized beam, unpolarized targets; (2) spindepolarization $D_{n n}$ and -rotation (the other $D_{i j}$ ) measurements with polarized beam, unpolarized targets; and (3) $A_{o o o n}, A_{o n n n}, \mathrm{~A}_{o o o, n n}$, and $\mathrm{A}_{o o n, n n}$ measurements using unpolarized/polarized beam and polarized targets. For the latter phase of the experiment we intend to use our own polarized target which is currently under construction at The University of Texas (see section 2.4 of this report). The target vector $(P)$ and tensor $\left(P_{T}\right)$ polarizations for cylindrically symmetric targets arc related at thermodynamic equilibrium (using the equal spin temperature (EST) 
theorem], by

$$
P_{T}=2-\sqrt{4-3 P^{2}}
$$

EST is expected to be valid for the polarized Li targets. ${ }^{10}$ We will use NMR to determine target vector polarization, as well as a $\vec{p}+\vec{p}$ monitor polarimeter of a design much the same as that used in EXP955. Beam line polarimeters will also provide precise information about beam polarization. We will have to do some developmeat runs using $\mathrm{LiH}$ "chunks" well in advance of the experiment to insure that a target made of "chunks" has sufficient homogeneity so as not to cause resolution problems. Effective target thickness will be monitored much more carefully than in EXP955. The contribution from ${ }^{7} \mathrm{Li}$ will include elastic scattering and inelastic $\left(p, p^{\prime}\right)$ to the first excited state $\left(\frac{1}{2}^{-}, 0.48 \mathrm{MeV}\right)$. The runs with the ${ }^{7} \mathrm{Li}$ target will allow this contribution to the ${ }^{6} \mathrm{Li}$ data to be determined and accurately subtracted.

\section{References}

[1] Proceedings of the LAMPF Workshop on Physics with Polarized Nuclear Targets, Los Alamos National Laboratory, LAMPF Conference Report LA10772-C (1986), edited by G. Burleson, W. Gibbs, G. Hoffmann, J. J. Jarmer, and N. Tanaka.

[2] G. W. Hoffmann et al., Phys. Rev. Lett. 65, 3096 (1990).

[3] L. Ray et al., Phys. Rev. C37, 1169 (1988).

[4] S. Cohen and D. Kurath, Nucl. Phys. 73, 1 (1965).

[5] G. L. Payne and B. P. Nigam, Phys. Rev. C21, 1177 (1980).

[6] J. B. J. M. Lanen et al., Phys. Rev. Lett. 63, 2793 (1989).

[7] L. Ray and G. W. Hoffmann, Phys. Rev. C31, 538 (1985).

[8] G. W. Hoffmann et al., Phys. Rev. C41, 1651 (1990).

[9] J. Bystricky, F. Lehar, and P. Winternitz, J. Phys. (Paris) 39, 1 (1978).

[10] R. Tacik et al., Phys. Rev. Lett. 63, 1784 (1989). 


\section{Figure Captions}

Figure 1: RIA-DWBA predictions for $500 \mathrm{MeV} \vec{p}+{ }^{6} \overrightarrow{\mathrm{Li}}$ elastic scattering differential cross section and spin observables as explained in the text.

Figure 2: Comparison of RIA-DWBA (shaded bands) and NRIA-DWBA (dashed curves) results for $500 \mathrm{MeV} \vec{p}+{ }^{6} \overrightarrow{\mathrm{Li}}$ elastic scattering differential cross section and spin observables as explained in the text.

Figure 3: RIA-DWBA theoretical calculations for $500 \mathrm{MeV} \vec{p}+{ }^{6} \overrightarrow{\mathrm{Li}}$ elastic scattering target spin observables, $A_{o o o n}$ and $A_{\text {oonn }}$, showing the individual contributions of the pseudoscalar (dashed curves), space-like vector (dotted curves), axial-vector (long-dashed-dotted curves), and tensor (short-dashed-dotted curves) components of the isoscalar $N N$ invariant amplitudes. The results using the full NN invariant amplitude are shown by the solid curves. Further details are in the text. 

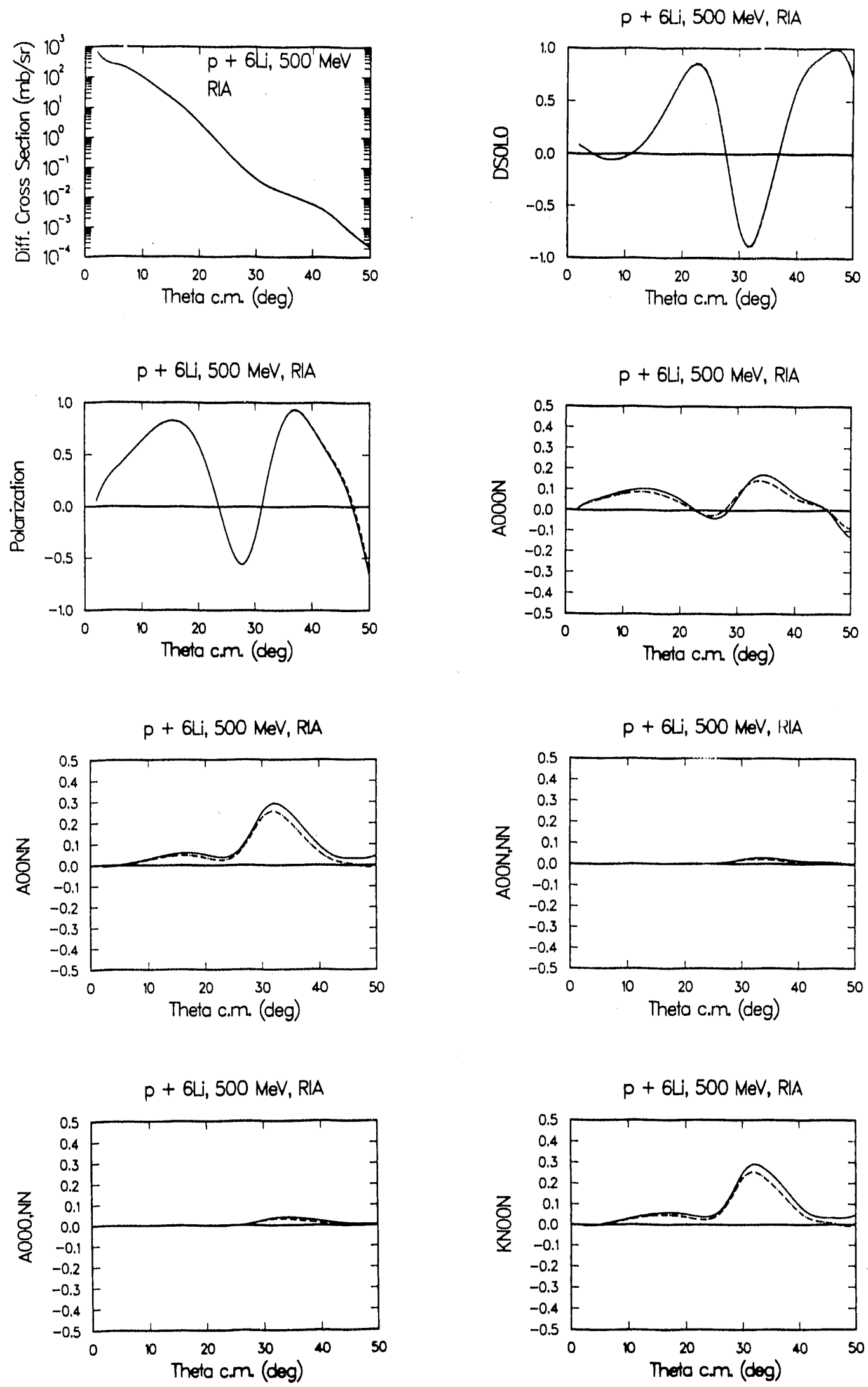

Figure 1 

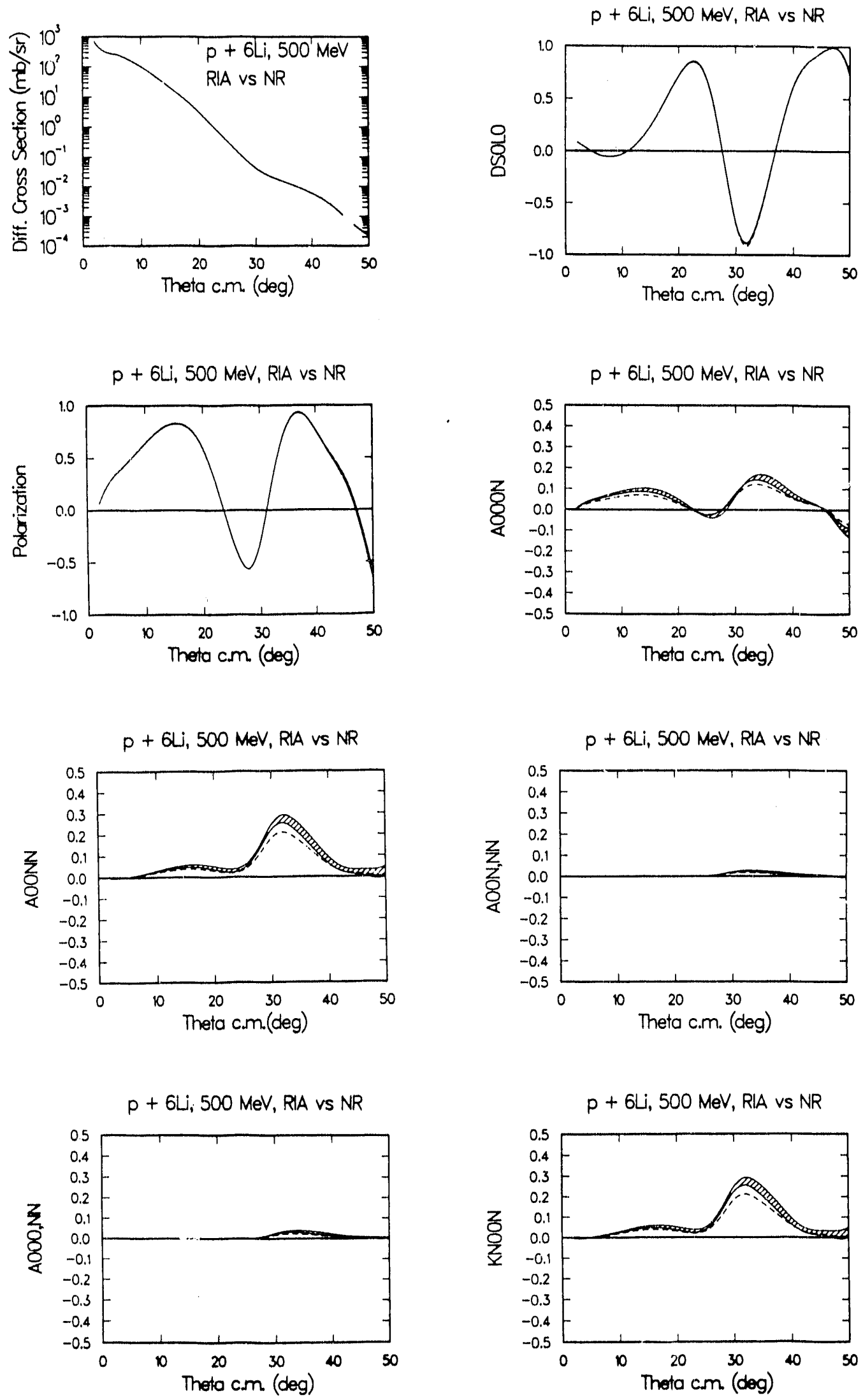

Figure 2 

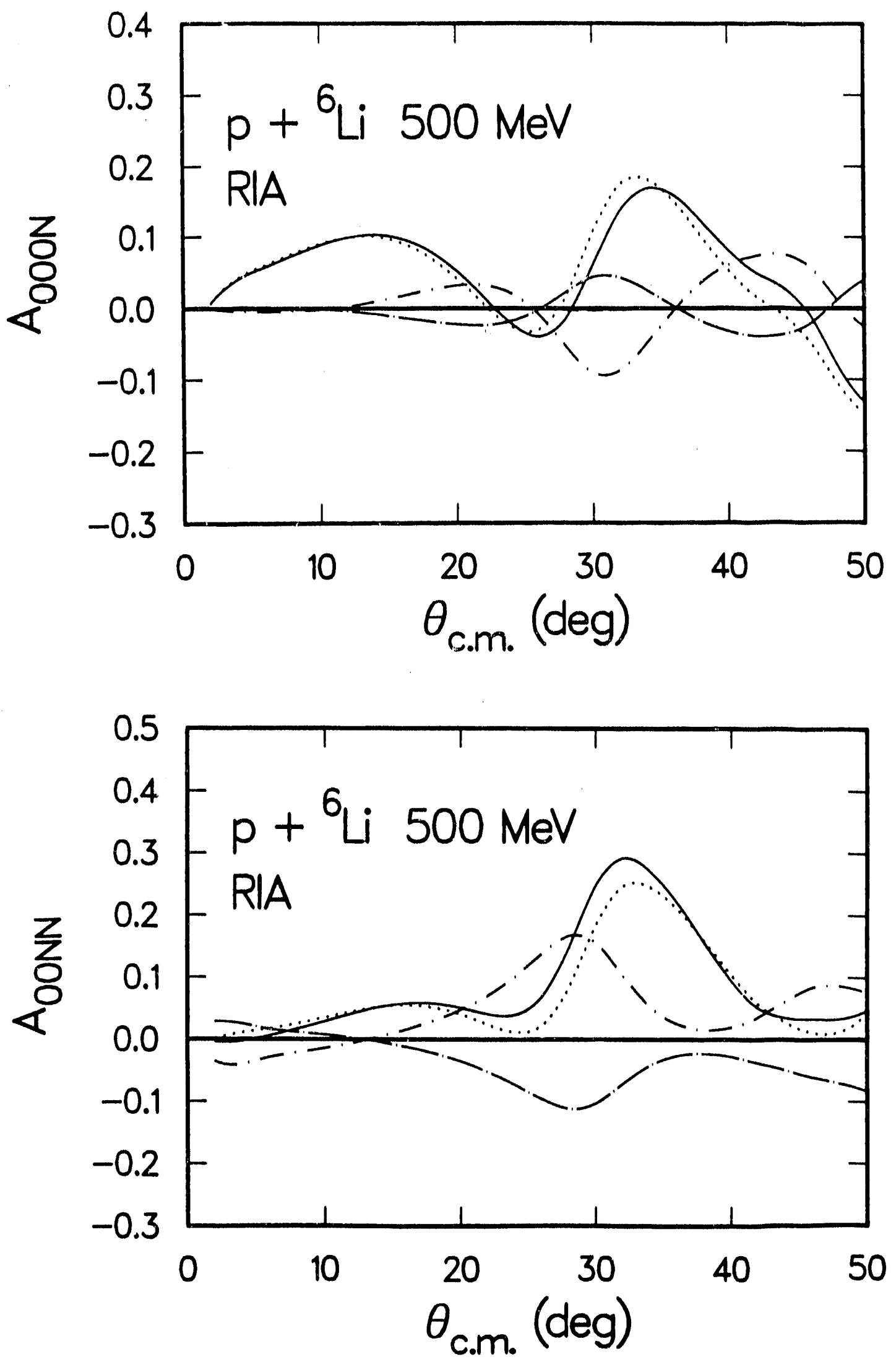

Figure 3 


\title{
4 Theoretical Work
}

\author{
(L. Ray; OSU; Maryland; Georgia)
}

The theoretical work discussed here is mainly related to nucleon-nucleus reactions at medium energies and continues to be directly associated with the experimental program in proton-nucleus scattering and with the new projects discussed in the previous two sections. The main efforts will include: continued improvements in the reaction models for polarized nucleon - polarized nucleus scattering; initiating a comprehensive calculation which combines medium effects, off-shell dependence and full-folding in the nonrelativistic (NR) optical potential; microscopic analysis of quasi-free $\Delta$ production in nuclei; and studies of $N N$ potential models derived from chiral invariant effective Lagrangians.

\subsection{Review of Nonrelativistic and Relativistic Descriptions of Proton-Nucleus Scattering}

We have completed a detailed review article ${ }^{1}$ in which nonrelativistic and relativistic scattering formalisms for nucleon-nucleus scattering were discussed and applications given for many cases of proton elastic scattering at medium energies. This review comes at a time when parameter free theoretical predictions based on nonrelativistic and relativistic dynamics are both beginning to provide reasonable descriptions of the data.

Research in this field continues to attract widespread theoretical interest. The 40 year history of this field attests to the fact that microscopic studies of a single nucleon's interaction with a nucleus relate to a fundamental problem in nuclear physics: a description of the complicated many-body scattering process (nucleon + nucleus) solely in terms of the underlying nucleon + nucleon (NN) dynamics and the structure of the target nucleus.

In the review article we included pedagogical discussion of the theory and showed predictions for the following scattering models: (1) the nonrelativistic, density-dependent (NRDD) model, ${ }^{2}(2)$ the nonrelativistic full-folding model, ${ }^{3}(3)$ the nonrelativistic Paris-Hamburg $g$-matrix optical model ${ }^{4}(4)$ the relativistic impulse approximation (RIA) model, ${ }^{5}$ and (5) the relativistic meson exchange model (IA2). ${ }^{6}$ The principal assumptions and ingredients of each nonrelativistic and relativistic scattering calculation are summarized in Tables I and II, respectively.

For the relativistic calculations using the IA2 interaction the positive energy projections of the $\mathrm{NN}$ relativistic $t$-matrix were directly constrained by phase shift analysis. The calculations using the NRDD interaction were based on the density dependent interaction ${ }^{2}$ where the $t$-matrix was normalized to the newer, SP89 phase shift solution of Arndt. -- 
The relativistic IA2 calculations used the nuclear densities described in Ref. 5. Briefly these correspond to the known proton vector density determined by electron scattering, Hartree-Fock-Bogoliubov neutron densities for the neutron vector density, and relativistic mean field densities for the scalar and tensor densities. The NRDD calculations and the NR calculations based on the Paris-Hamburg $g$ matrix 4 used the above proton and neutron vector densities. For the $200 \mathrm{MeV}$, full-folding predictions ${ }^{3}$ the prolon and neutron density matrices were computed from Woods-Saxon single particle eigenstates which were fitted to empirical charge density root-mean-square (rms) radii and single particle energies.

Anomalous magnetic moment (AMM) terms were included in each of the IA2 calculations. The electromagnetic spin-orbit (EMSO) potential was included in the NRDD predictions for $800 \mathrm{MeV}$ for each target and for ${ }^{40} \mathrm{Ca}$ and ${ }^{208} \mathrm{~Pb}$ at $500 \mathrm{MeV}$. EMSO effects are not expected to be important for the remaining cases. Two-body correlation contributions were also included in the NRDD calculations. The relativistic optical potential correlation contributions were not included in the IA 2 or the RIA calculations since these effects are small.

In Figs. 1 - 9 the relativistic and NR model predictions for $p+{ }^{16} \mathrm{O},{ }^{40} \mathrm{Ca}$ and ${ }^{208} \mathrm{~Pb}$ at 200,500 and $800 \mathrm{MeV}$ are compared with differential cross section $(d \sigma / d \Omega)$, analyzing power $\left(\mathrm{A}_{\mathrm{y}}\right)$ and spin rotation $(\mathrm{Q})$ data. The NR density dependent results ${ }^{2}$ are indicated by the solid curves. The NR results based on the Paris-Hamburg $g$-matrix ${ }^{4}$ are shown by the dashed-dotted curves. The NR fullfolding predictions ${ }^{3}$ are represented by the dotted lines, and the relativistic IA2 observables ${ }^{6}$ are shown by the dashed curves.

At $200 \mathrm{MeV}$ the NR calculations using the Paris-Hamburg $g$-matrix give about the right amount of structure for the differential cross sections and correctly account for the damping of the diffractive structure for ${ }^{40} \mathrm{Ca}$ and ${ }^{208} \mathrm{~Pb}$ near $q \cong 2 \mathrm{fm}^{-1}$. The NR full-folding results provide a reasonable description of the $p+{ }^{16} \mathrm{O}$ and ${ }^{40} \mathrm{Ca}$ differential cross sections but tend to have a little too much structure. The IA 2 differential cross sections are fairly good but also display too much structure, particularly for $p+{ }^{208} \mathrm{~Pb}$. The analyzing powers predicted by all three models are qualitatively successful; all three models provide good descriptions of the spin rotation data. Overall, there is no strong preference for either the relativistic or the NR approaches at this energy.

The relativistic IA2 and NRDD-EMSO curves for $p+{ }^{16} \mathrm{O},{ }^{40} \mathrm{Ca}$ and ${ }^{208} \mathrm{~Pb}$ at $500 \mathrm{MeV}$ are shown in comparison with data in Figs. 4 - 6. The IA2 predictions accurately reproduce the angular positions of the diffractive minima and the overall slope of the differential cross section data. The NRDD cross sections have the correct diffractive shapes; however, they are a little too small in magnitude and are shifted inward to smaller scattering angles relative to the data. The NRDDEMSO model provides the better description of the analyzing power data. The NRDD-EMSO model predictions for the spin rotation parameter $Q$ are larger than the IA2 predictions for $q \leq 1-2 \mathrm{fm}^{-1}$ and are in slightly better agreement with the data. Both models fail quantitatively to reproduce the forward angle $A_{y}$ and 
$Q$ data $\mathrm{f}_{\mathrm{i}} q \leq 1.5 \mathrm{fm}^{-1}$; the calculated analyzing powers tend to be too large while the spin rotations are too small. Overall, both models provide qualitative descriptions of the $500 \mathrm{MeV}$ data.

Historically, $500 \mathrm{MeV}$ was the energy where relativistic theories were most successful, especially for the RIA model. ${ }^{7,8}$ In view of the results shown in Figs. 4 6 , this is certainly not the case for sophisticated NR scattering models and relativistic models which use covariant meson exchange theory. It turns out, in fact, that the success of the RIA model at $500 \mathrm{MeV}$ was to some extent fortuitous. The gross discrepancies between the NR model predictions and data at $500 \mathrm{MeV}$, which formed the basis of an important issue in the 1980's, are no longer a relevant concern.

At $800 \mathrm{MeV}$ both the NRDD-EMSO and the IA2 model predictions are in good, overall agreement with the data as shown in Figs. 7 - 9. The NR cross sections, however, display the characteristic problem with respect to angular positions of the minima and the overall magnitude and slope. The IA 2 cross sections display an incorrect mass dependence - too small in overall magnitude for ${ }^{16} \mathrm{O}$, roughly correct for ${ }^{40} \mathrm{Ca}$, and a little too large for ${ }^{208} \mathrm{~Pb}$. The $p+{ }^{16} \mathrm{O}$ analyzing powers and spin rotations of both theories display too much structure, particularly predictions of the IA2. The NR $p+{ }^{40} \mathrm{Ca} \mathrm{A} \mathrm{A}_{y}$ results compare well with the data at forward angles but underestimate the data for $q \geq 2 \mathrm{fm}^{-1}$. Both calculated $p+{ }^{40} \mathrm{Ca}$ spin rotation curves are too deep at the minima. Each model provides quantitative descriptions of the $p+{ }^{208} \mathrm{~Pb} \mathrm{~A}_{\mathrm{y}}$ data, except at the larger angles. The $p+{ }^{208} \mathrm{~Pb}$ IA 2 spin rotations tend to be too small in magnitude.

The results shown in Figs. 1 - 9 make it apparent that a clear phenomenological preference does not exist for either the relativistic or the nonrelativistic approaches. Both methors are capable of describing qualitatively the body of medium energy proton-nucleus elastic scattering data, as represented by these 9 specific examples.

The curious result to come out of all this is the similarity between the effects on the predicted observables of medium and off-shell corrections in the NR approach and the virtual $N \bar{N}$ pair contributions in the relativistic IA2 model. We have no explanation to offer for this. Both the medium corrections and the virtual NN pair contributions are roughly proportional to the nuclear density squared. However, there is no obvious relation between the strengths of these various terms, which originate from very different sources, i.e. Pauli blocking, binding energy, and off-shell dependences in the two-component Pauli representation, and coupling to negative energy states in the four-component Dirac representation. The similarity which is presently found may only be coincidental and future efforts would most likely be best spent further implementing the NR formalism and providing a better understanding of the virtual $N \bar{N}$ pair process in the relativistic models.

We will continue to develop the nonrelativistic many-body scattering models in order to learn how far traditional nonrelativistic theories can go in describing nuclear phenomenon. Systematic discrepancies, if they occur in the final analysis, probably indicate missing physics in the theory, e.g. Lorentz invariance, many- 
body forces, quark-gluon effects, etc. We must, of course, be careful to distinguish between failure of the nonrelativistic many-body approach itself, and inadequacy of some particular numerical application.

Relativistic quantum field theories with nucleons and mesons and relativistic nucleon-nucleus scattering models should also be pursued further, as such work provides important tools for dealing with the relativistic nuclear many-body problem and indicates the level at which relativistic effects might manifest themselves in nuclear systems. The relativistic scattering models reviewed in Ref. 1 provide an important step along the way towards a covariant description of nuclear physics.

\section{References}

[1] L. Ray, G. W. Hoffmann, and W. R. Coker, Physics Reports (1991), in press.

[2] L. Ray, Phys. Rev. C 41, 2816 (1990).

[3] H. F. Arellano, F. A. Brieva, and W. G. Love, Phys. Rev. C 41, 2188 (1990); (errata) Phys. Rev. C 42, 1782 (1990).

[4] H. V. von Geramb, in The Interaction Between Medium Energy Nucleons in Nuclei, Proceedings of the Workshop on the Interactions Between Medium Energy Nucleons in Nuclei, AIP Conf. Proc. No. 97, edited by H. O. Meyer (AIP, New York, 1983), p. 44.

[5] L. Ray and G. W. Hoffmann, Phys. Rev. C 31, 538 (1985).

[6] N. Ottenstein, S. J. Wallace and J. A. Tjon, Phys. Rev. C 38, 2272 (1988);

J. A. Tjon and S. J. Wallace, Phys. Rev. C 36, 1085 (1987).

[7] J. Shepard, J. A. McNeil and S. J. Wallace, Phys. Rev. Lett. 50, 1443 (1983).

[8] B. C. Clark, S. Hama, R. L. Mercer, L. Ray, and B. D. Serot, Phys. Rev. Lett. 50, 1644 (1983). 


\section{Figure Captions}

Figure 1: Best nonrelativistic and relativistic theoretical predictions for $p+{ }^{16} \mathrm{O}$ elastic scattering at $200 \mathrm{MeV}$ are compared with data. Predictions of the relativistic IA2 model (from Ref. 6), the NR Paris-Hamburg $g$-matrix, ${ }^{4}$ and the NR full-folding model of Ref. 3 are indicated by the dashed, dashed-dotted, and dotted curves, respectively.

Figure 2: Same as Fig. 1, except $p+{ }^{40} \mathrm{Ca}$ at $200 \mathrm{MeV}$.

Figure 3: Same as Fig. 1, except $p+{ }^{208} \mathrm{~Pb}$ at $200 \mathrm{MeV}$.

Figure 4: Best nonrelativistic and relativistic theoretical predictions for $p+{ }^{16} \mathrm{O}$ elastic scattering at $500 \mathrm{MeV}$ are compared with data. Predictions of the relativistic IA2 model (from Ref. 6) and the NRDD-EMSO model are indicated by the dashed and solid curves, respectively.

Figure 5: Same as Fig. 4, except $p+{ }^{40} \mathrm{Ca}$ at $500 \mathrm{MeV}$.

Figure 6: Same as Fig. 4, except $p+{ }^{208} \mathrm{~Pb}$ at $500 \mathrm{MeV}$.

Figure 7: Same as Fig. 4, except $p+{ }^{16} \mathrm{O}$ at $800 \mathrm{MeV}$.

Figure 8: Same as Fig. 4, except $p+{ }^{40} \mathrm{Ca}$ at $800 \mathrm{MeV}$.

Figure 9: Same as Fig. 4, except $p+{ }^{208} \mathrm{~Pb}$ at $800 \mathrm{MeV}$. 


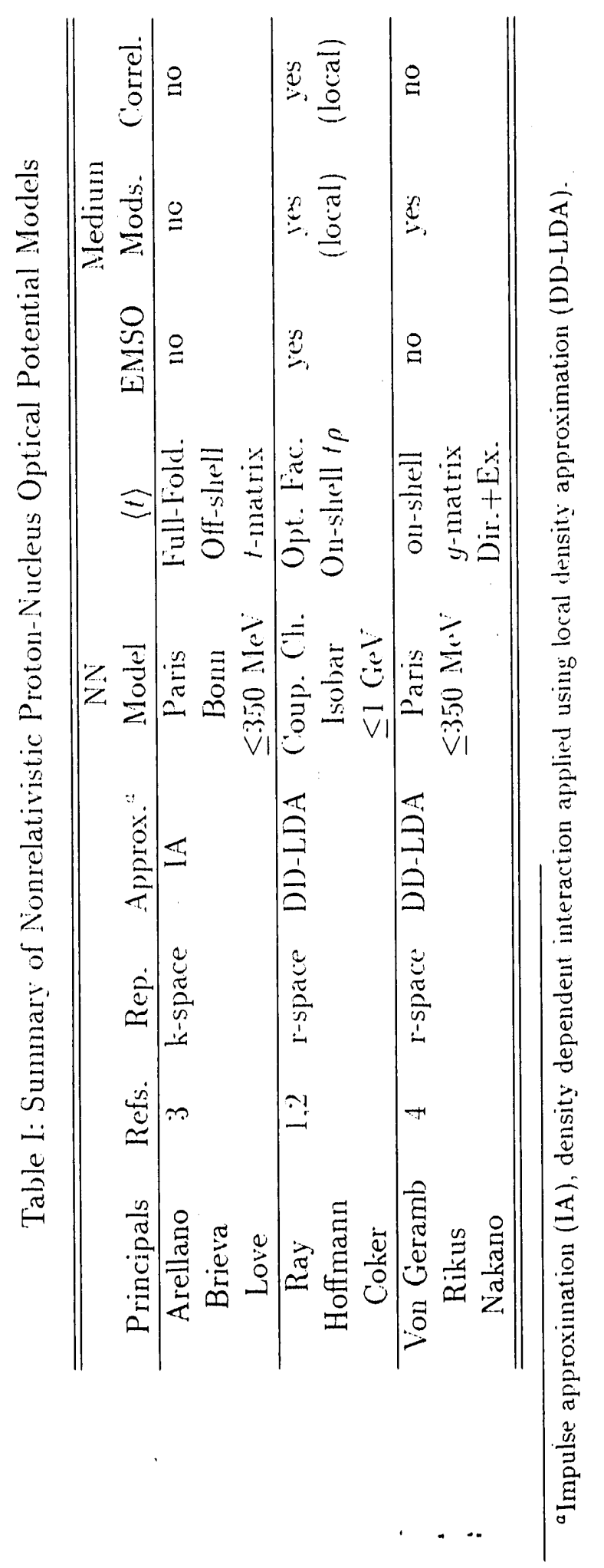




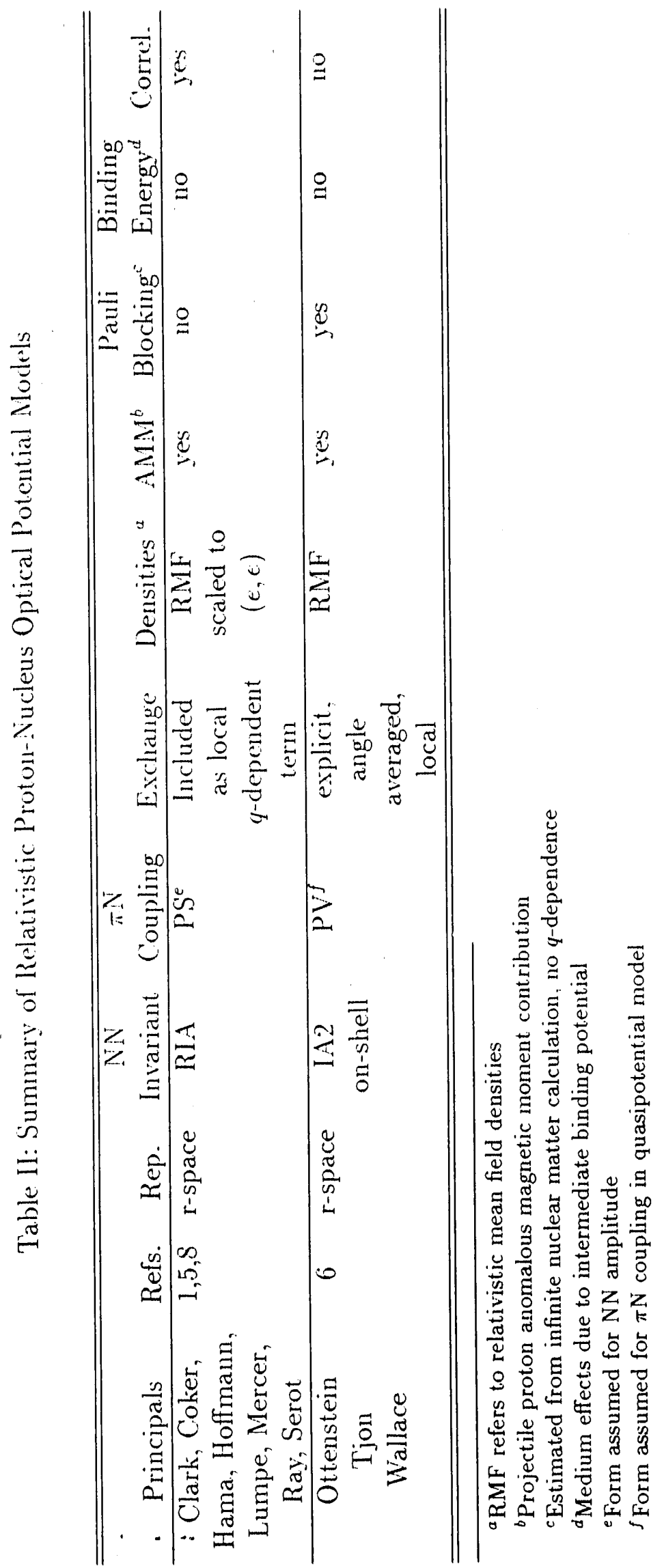

78 

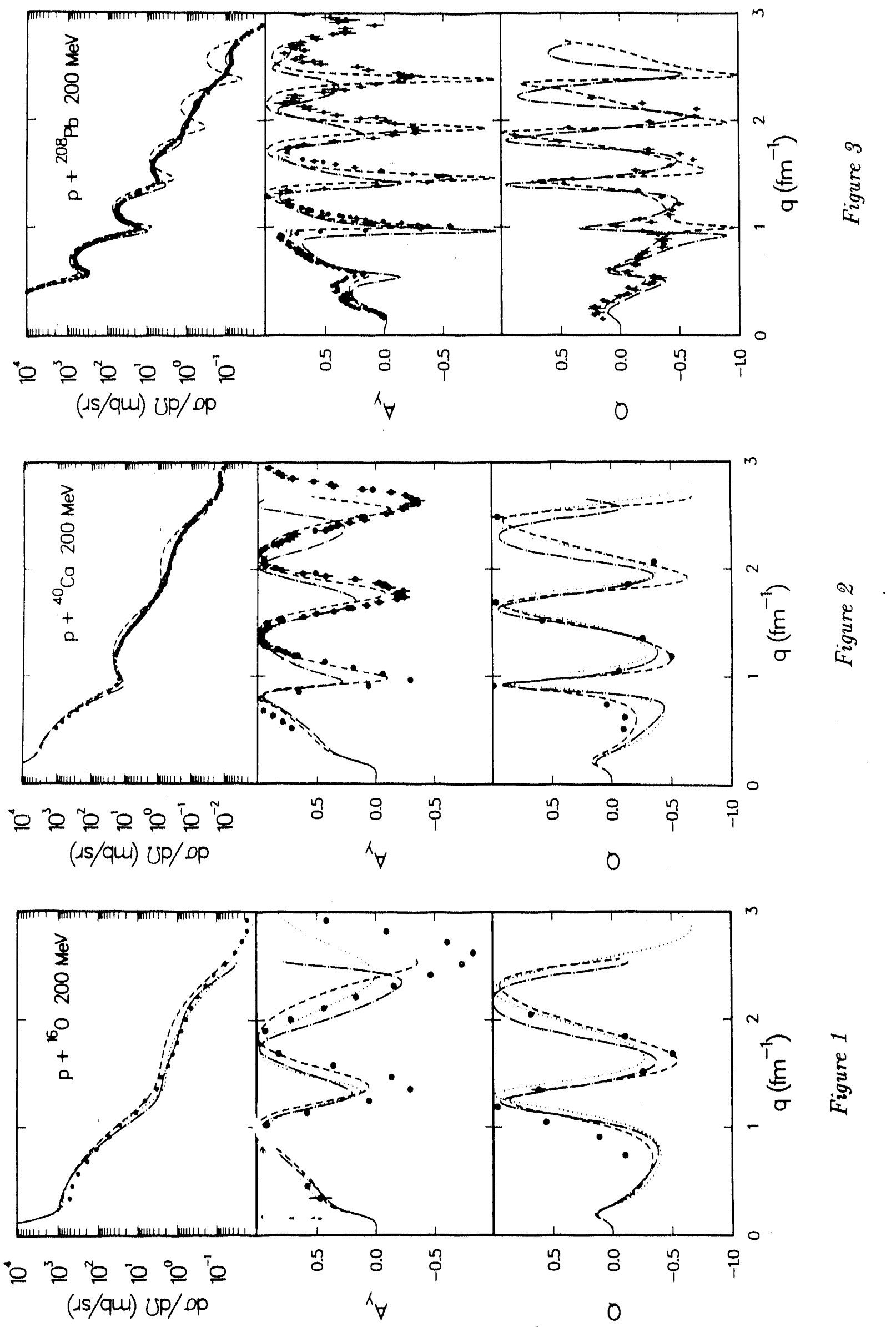

79 

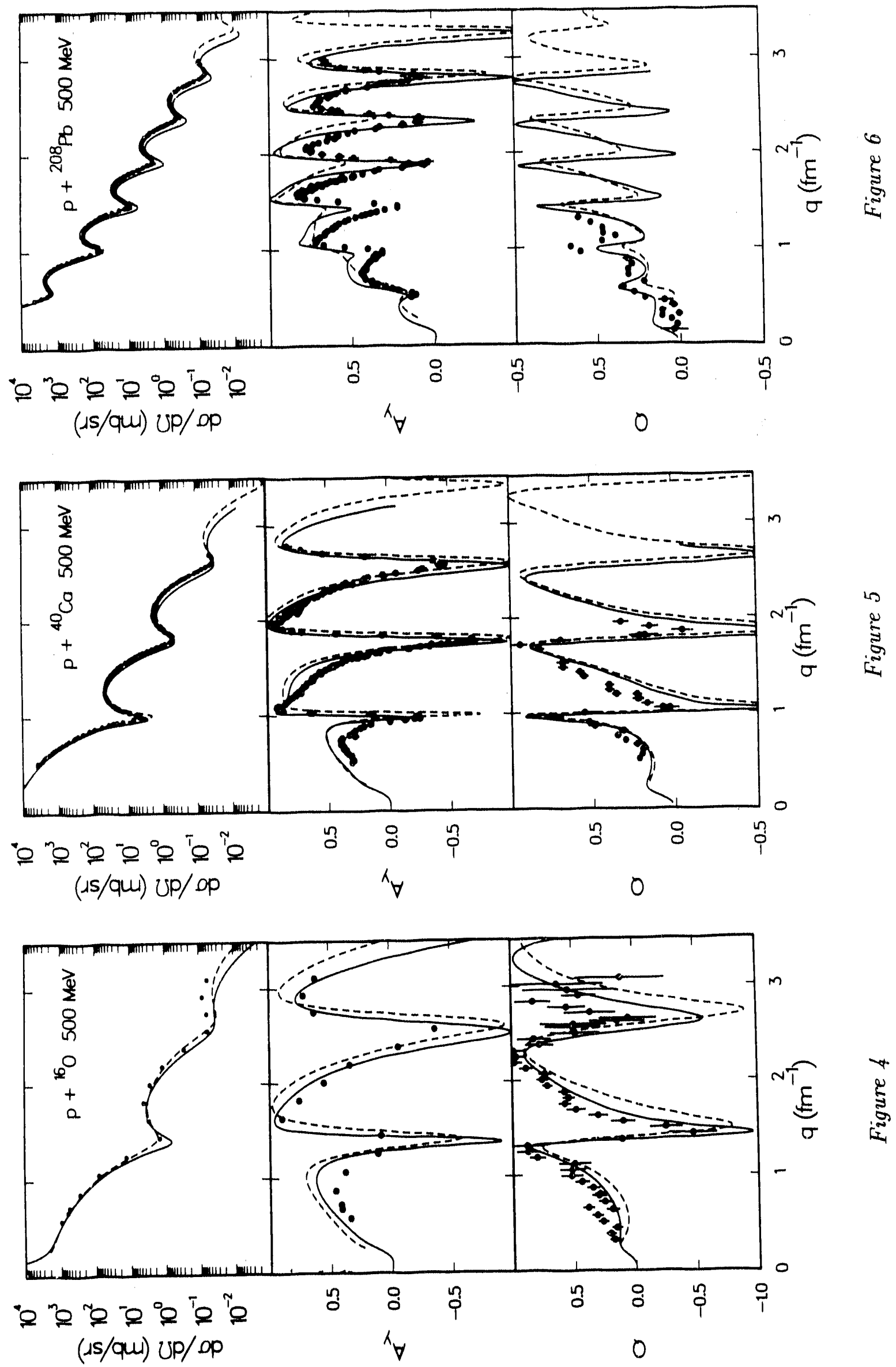

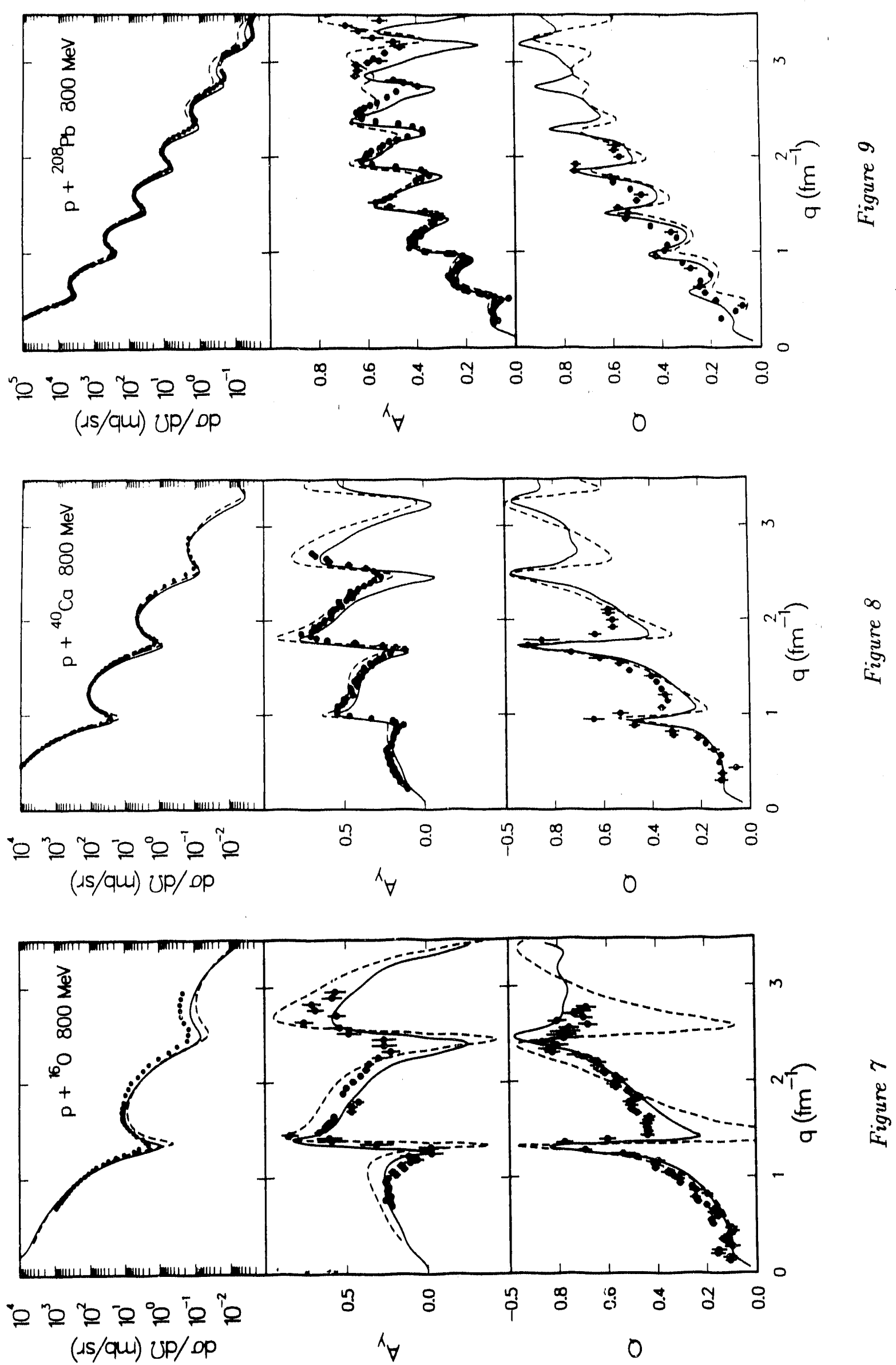


\subsection{Nuclear Structure and Mediam Effects in $\vec{p}+{ }^{13} \overrightarrow{\mathrm{C}}$ Elastic Scattering}

Rclativistic and nonrelativistic distorted wave Born approximation (DWBA) calculations $^{1,2}$ describe the general features of the $500 \mathrm{MeV} \vec{p}+{ }^{13} \overrightarrow{\mathrm{C}} A_{\text {ooon }}$ and $A_{\text {oonn }}$ data but do not fit details of the smaller angle data around $20^{\circ} \mathrm{c} . \mathrm{m}$. In the analysis of Ref. 2 impulse approxination values for the $\mathrm{NN}$ effective interaction were used and a nuclear structure model containing a pure $1 p_{\frac{1}{2}}$ valence neutron plus ${ }^{12} \mathrm{C}$ core was assumed. In this work the sensitivities of the predictions to medium corrections ${ }^{3}$ and shell-model configuration mixing ${ }^{4}$ were studied using the nonrelativistic DWBA reaction model.

For this application the $p+{ }^{13} \mathrm{C}$ optical potential was separated into $J=0$ and $J>0$ components, where

$$
U_{\mu^{\prime} \mu}^{o p t}=\sum_{i=1}^{A}\left\langle\Phi_{g s, \mu^{\prime}}\left|t_{0 i}\right| \Phi_{g s, \mu}\right\rangle=U_{J=0}^{o p t} \delta_{\mu^{\prime} \mu}+U_{\mu^{\prime} \mu, J>0}^{o p t}
$$

$J$ is the total angular momentum transfer, $\Phi_{g s, \mu}$ is the ${ }^{13} \mathrm{C}$ ground state wave function, $\mu\left(\mu^{\prime}\right)$ is the in $a$ ? (final) spin projection of the target nucleus, $t_{0 i}$ is the NN effective interaction $i$ ialatrix, and $A$ is the number of target nucleons. In the second quantization representation $\sum_{i} t_{0 i}$, is given by

$$
\hat{F}=\sum A_{J M T T_{3}}^{\alpha \beta}\left[a_{\alpha}^{\dagger} \otimes a_{\tilde{\beta}}\right]_{J M T T_{3}}
$$

where

$$
\begin{aligned}
A_{J M T T_{3}}^{\alpha \beta}= & \sum_{m_{\alpha} m_{\beta}} \sum_{t_{\alpha} t_{\beta}}(-)^{j_{\beta}-m_{\beta} \cdot \frac{1}{3}-t_{\beta}}\left(j_{\alpha} m_{\alpha} j_{\beta}-m_{\beta} \mid J M\right)\left(\frac{1}{2} t_{\alpha} \frac{1}{2}-t_{\beta} \mid T T_{3}\right) \\
& \times\left\langle u_{\alpha, m_{\alpha}, t_{\alpha}}\left|t_{01}\right| u_{\beta, m_{\beta}, t_{\beta}}\right\rangle,
\end{aligned}
$$

$\left[a_{t}^{\dagger} \otimes a_{\bar{\beta}}\right]_{J M T T_{3}}$ produces a particle-hole excitation in subshells $\alpha=\left\{n_{\alpha}, \ell_{\alpha}, j_{\alpha}\right\}$ and $\beta=\left\{n_{\beta}, \ell_{\beta}, j_{\beta}\right\}$ coupled to total = ngular momentum and isospin $(J, M)$ and $\left(T, T_{3}\right)$, respectively, and $u_{n \ell j m t}$ is a single particle state. In this representation the $J>0$ part of the optical potential becomes

$$
\begin{aligned}
U_{\mu^{\prime} \mu, J>0}^{o p t}= & \sum_{\alpha \beta} \sum_{J>0, M T T_{3}} A_{J M T T_{3}}^{\alpha \beta}\left(J_{i} \mu J M \mid J_{f} \mu^{\prime}\right)\left(T_{i} T_{i 3} T T_{3} \mid T_{f} T_{f 3}\right) \\
& \times\left\langle J_{f} T_{f}\left\|\left[a_{\alpha}^{\dagger} \otimes a_{\tilde{\beta}}\right]_{J T}\right\| J_{i} T_{i}\right\rangle
\end{aligned}
$$

where $\left(J_{i}, \mu\right)$ and $\left(T_{i}, T_{i 3}\right)$ denote the total angular momentum and isospin (plus $z$ projections) of the target nucieus in the initial state. Similar quantities are defined for the final nuclear state. For elastic scattering from ${ }^{13} \mathrm{C} J_{i}=J_{f}=\frac{1}{2}, T_{i}=T_{f}=\frac{1}{2}$, and $T_{i 3}=T_{f 3}=-\frac{1}{2}$. The doubly reduced (in spin and isospin) matrix elements 
in Eq. (4.4) are proportional to the reduced density matrix elements (RDMEs), $\phi_{J T}^{f i}(\alpha \beta)$, obtained in shell-model calculations ${ }^{4}$ where

$$
\phi_{J T}^{f_{i}}(\alpha \beta)=\sqrt{\frac{2\left(2 j_{\alpha}+1\right)}{(2 J+1)(2 T+1)}}\left\langle J_{f} T_{f}\left\|\left[a_{\alpha}^{\dagger} \otimes a_{\dot{\beta}}\right]_{J T}\right\| J_{i} T_{i}\right\rangle .
$$

The $J=0$ part of $U_{\mu^{\prime} \mu}^{\text {opt }}$ was assumed to be the Schrödinger equivalent of the Dirac phenomenological optical potential for $500 \mathrm{MeV} p+{ }^{12} \mathrm{C}$ elastic scattering plus the scalar and time-like vector parts of the relativistic impulse approximation optical potential for the $1 p_{\frac{1}{2}}$ valence neutron state. The NN density dependent $t$-matrix calculated in Ref. $3^{2}$ was used for $t_{0 i}$.

In Refs. 1 and 2 the nuclear structure model was not constrained by the $M 1$ elastic form factor measured in back angle electron scattering. ${ }^{5}$ 'Therefore the Cohen and Kurath (CK) (8-16)2BME $p$-shell model ${ }^{4}$ and an empirical radial wave function were adopted and used to fit the $M 1$ form factor. For comparison, calculations were also done assuming a pure $1 p_{\frac{1}{2}}$ neutron configuration with harmonic oscillator radial wave function where the oscillator parameter was adjusted to reproduce the zero of the $M 1$ form factor at $q=1.04 \mathrm{fm}^{-1}$. The two radial wave functions used here and the corresponding $M 1$ form factors are shown in Figs. 1 and 2 , respectively.

The results for $A_{\text {ooon }}$ and $A_{\text {oonn }}$ are shown in Fig. 3. The predictions assuming the pure $1 p_{\frac{1}{2}}$ model with harmonic oscillator radial wave function and the NN $t$ matrix fixed at the zero-density value (impulse approximation) are shown by the dotted curves. The results using the $\mathrm{CK}$ wave functions with and without density dependence in the NN $t$-matrix are shown by the solid and dashed curves, respectively. Density dependent effects are small for this case while the differences due to the choice of nuclear structure models are greater, particularly for $A_{\text {ooon }}$ at the larger angles. While the overall structures of the two sets of target spin observable data are described by these predictions the peaks near $20^{\circ} \mathrm{c} . \mathrm{m}$. are inadequately explained. Predictions based on the relativistic impulse approximation DWBA model do not fit the forward angle data either. ${ }^{2}$

The present work demonstrates that medium corrections and nuclear structure effects of the type considered here are unimportant for this case, at least in the forward angle region around $20^{\circ} \mathrm{c} . \mathrm{m}$. where discrepancies between theory and experiment are greatest.

\section{Future Work}

Preliminary calculations ${ }^{6}$ using a generalized Dirac coupled-channels computer program indicate that the DWBA is accurate for this reaction. Further investigation of the distorted wave approximation and coupled-channels effects for this case should be completed fairly soon.

A group at the Univ. of Maryland ${ }^{7}$ has recently developed a two-component Pauli representation for the refativistic IA2 $\mathrm{NN} t$-matrix. The resulting $\mathrm{NN}$ am- 
plitudes have the usual Wolfenstein form and are density dependent. With this form of the relativistic IA2 interaction and the reaction model in Ref. 1 we can readily obtain the IA 2 predictions for $\vec{p}+{ }^{13} \overrightarrow{\mathrm{C}}$ elastic scattering. This will provide an important test of the spin-transfer components of the IA2 model.

Finally, the importance of full-folding and off-shell effects in proton-nucleus elastic scattering at forward angles suggests that these small corrections might also be important for $\vec{p}+{ }^{13} \overrightarrow{\mathrm{C}}$. We plan to initiate this calculation following a similar effort for spin zero targets.

\section{References}

[1] L. Ray et al., Phys. Rev. C 37, 1169 (1988).

[2] G. W. Hoffmann et al., Phys. Rev. Lett. 65, 3096 (1990).

[3] L. Ray, Phys. Rev. C 41, 2816 (1990).

[4] S. Cohen and D. Kurath, Nucl. Phys. 73, 1 (1965).

[5] R. S. Hicks et al., Phys. Rev. C 26, 339 (1982).

[6] B. C. Clark and R. L. Mercer, private communication.

[7] S. J. Wallace, private communication.

\section{Figure Captions}

Figure 1: Radial wave functions for the $1 p$ state used in the DWBA calculations. The harmonic oscillator wave function corresponding to the pure $1 p_{\frac{1}{2}}$ single neutron configuration is shown by the dashed curve while the solid curve indicates the empirical wave function used with the CK shell-model.

Figure 2: Elastic $M 1$ form factors (squared) in ${ }^{13} \mathrm{C}$ corresponding to the $1 p_{\frac{1}{2}}$ single neutron configuration with harmonic osciilator wave function (dashed curve) and the CK shell-model with an empirical radial wave function (solid curve). The form factor is expressed in units of nuclear magnetons.

Figure 3: Nonrelativistic DWBA predictions for $A_{\text {ooon }}$ (upper part) and $A_{\text {oonn }}$ (lower part) for $497.5 \mathrm{MeV} \vec{p}+{ }^{13} \overrightarrow{\mathrm{C}}$ elastic scattering. Dotted, dashed and solid curves renzesent the results of calculations assuming the $1 p_{\frac{1}{2}}$ single neutron model (no medium corrections), the CK shell-model (no medium corrections), and the CK shell-model with medium corrections, respectively. Data are from Ref. 2. 

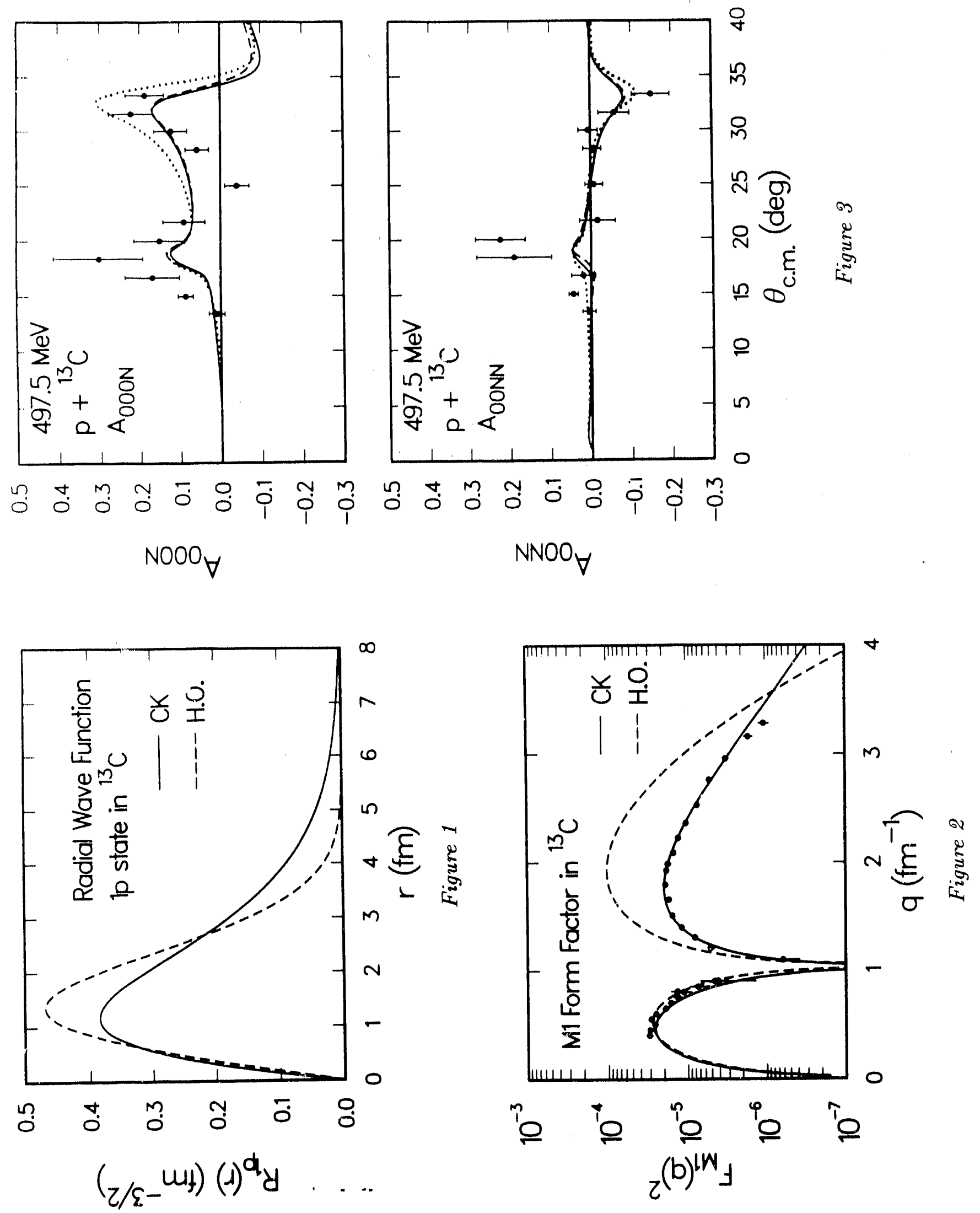


\subsection{Proton Elastic Scattering from Polarized ${ }^{3} \mathrm{He}$}

A group at TRIUMF has recently taken elastic scattering data for $\vec{p}+{ }^{3} \overrightarrow{\mathrm{He}}$ at several energies from 200 to $500 \mathrm{MeV}{ }^{1}$ Included are the differential cross section, the analyzing power, the target analyzing power $A_{\text {ooon }}$, and the spin correlation $A_{\text {oonn }}$. The data extend out to about $5 \mathrm{fm}^{-1}$ of momentum transfer. The nonrelativistic DWBA scattering model ${ }^{2}$ was used to generate predictions for the polarized target spin observables. The $p+{ }^{3} \mathrm{He}$ optical potential was separated into $J=0$ and $J=1$ components (where $J$ is the total angular momentum transfer); the $J=0$ part was described phenomenologically while the $J=1$ part was computed from the $s_{\frac{1}{2}}$ wave functions and the full, spin-dependent $N N t$-matrix. The $J=0$ part of the optical potential was obtained by fitting the $\vec{p}+{ }^{3} \mathrm{He}$ differential cross section and analyzing power data using Dirac phenomenology. ${ }^{3}$ This, in turn, provided the $J=0$ part of the $p+{ }^{3} \mathrm{He}$ elastic scattering amplitude and the distorted waves for use in the DWBA. The single particle wave functions were determined by fitting the measured $M 1$ form factor in ${ }^{3} \mathrm{He}$ (Ref. 4) where the ${ }^{3} \mathrm{He}$ nucleus was assumed to be an antisymmetrized product of $s_{\frac{1}{2}}$ ingle particle states. Meson exchange currents were ignored. The $\mathrm{NN} t$-matrix was used on-shell and was obtained from the Arndt SP89 phase shift solution.

The NR-DWBA predictions and data for $500 \mathrm{MeV} \vec{p}+{ }^{3} \overrightarrow{\mathrm{He}}$ elastic scattering are shown in Fig. 1. The predictions are reasonable for angles up to $40^{\circ} \mathrm{c.m}$. (about $3 \mathrm{fm}^{-1}$ momentum transfer) but differ significantly from the data at larger angles. The discrepancies might be due to unrealistic wave functions and/or NN $t$-matrices at large momentum transfer, meson exchange currents, multiple scattering effects, etc.

\section{References}

[1] O. Häusser, in the proceedings of the $7^{\text {th }}$ Int. Conf. on Polarization Phenomena in Nuclear Physics, Paris (1990).

[2] L. Ray, G. W. Hoffmann and W. R. Coker, Physics Reports (1991), in press.

[3] S. Hama, private communication.

[4] E. Hadjimichael et al., Phys. Rev. C 27, 831 (1983).

\section{Figure Caption}

Figure 1: Nonrelativistic DWBA predictions for $500 \mathrm{MeV} \vec{p}+{ }^{3} \overrightarrow{\mathrm{He}} A_{\text {ooon }}$ and $A_{\text {oonn }}$ in comparison with the data from Ref. 1. 


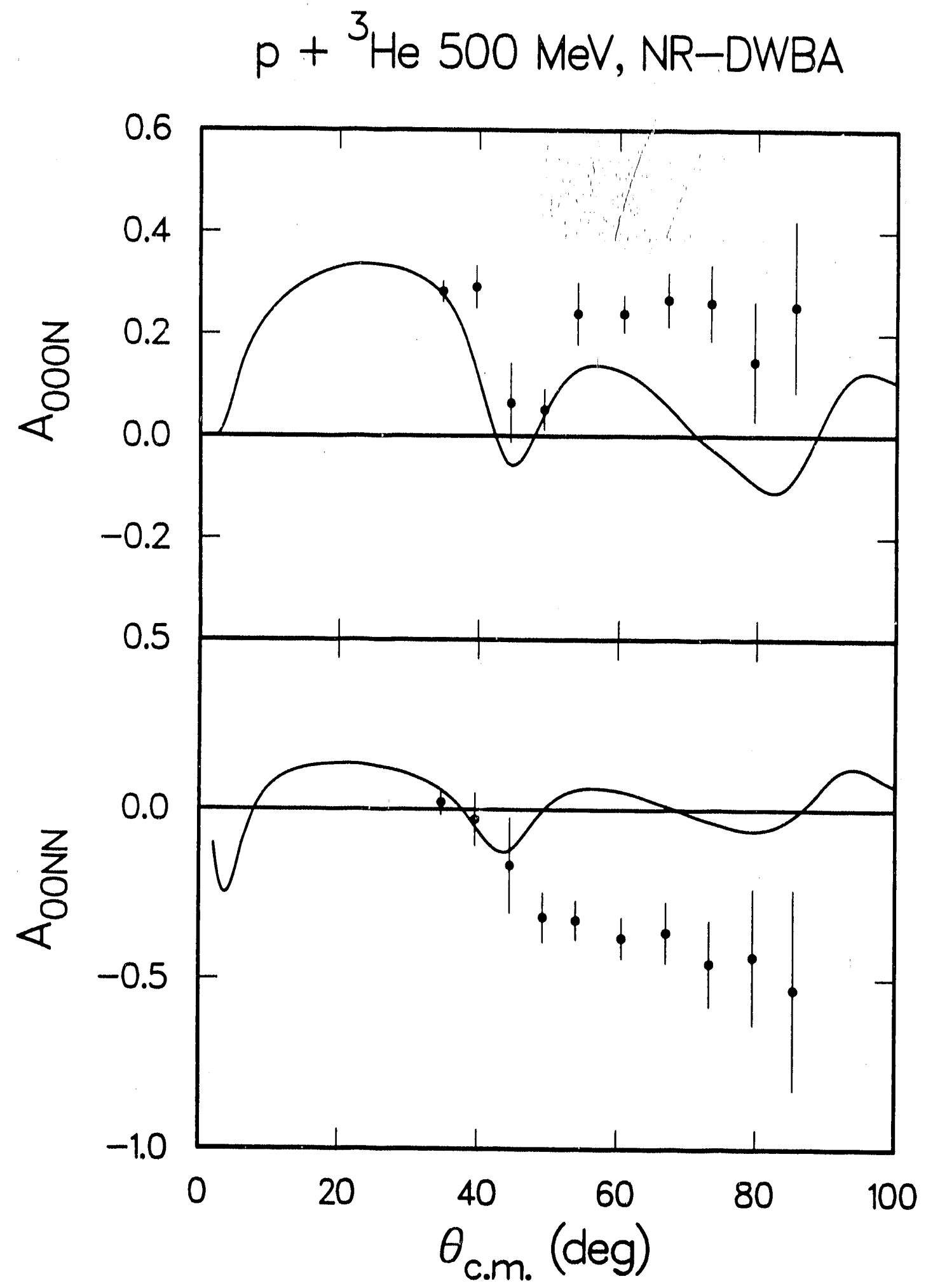

Figure 1 


\title{
4.4 New Theoretical Work
}

\author{
Full-Folding Density-Dependent Nonrelativistic Optical Potential
}

The success of density-dependent optical potential - Schrödinger equation descriptions of medium energy proton-nucleus elastic scattering data, ${ }^{1}$ combined with the larger than expected $\mathrm{NN} t$-matrix off-shell effects ${ }^{2,3}$ warrants a comprehensive investigation of full-folding optical potentials based on density-dependent $\mathrm{NN}$ effective interactions. It is necessary, however, to carry out calculations of this sort within a multiple scattering formalism which consistently treats projectile proton target nucleon antisymmetrization. It is customary to base numerical applications on either the Watson or KMT optical potential formalisms in which the identity of the projectile nucleon and target nucleon constituents is only approximately accounted for (i.e., Takeda and Watson ${ }^{4}$ procedure). In the KMT formalism, for example, Pauli blocking medium corrections cancel part of the second-order optical potential. This cancellation will be altered when the projectile is a proton or neutron.

In the near future calculations will be made which include medium corrections to the NN effective interaction, off-shell effects, target nucleon correlations, and projectile - target nucleon antisymmetrization, all within the framework of the fully antisymmetrized multiple scattering theory of Ref. 5. The NN density-dependent interaction model of Ref. 1 will be used for calculating medium corrections and for generating the off-shell NN $t$-matrix. The full-folding, optical potential - momentum space code of Ref. 2 will be used to do the complete, density-dependent calculations. This project will be carried out in collaboration with Prof. W. G. Love (Univ. of Georgia). Calculations for the canonical targets $\left({ }^{16} \mathrm{O},{ }^{40} \mathrm{Ca}\right.$, ${ }^{208} \mathrm{~Pb}$ ) from a few hundred $\mathrm{MeV}$ to $800 \mathrm{MeV}$ will be done. When the first-order medium effects are properly combined with second-order optical potential (correlation) corrections, electromagnetic spin-orbit (EMSO) potentials, and other smaller corrections, we will finally be in position to judge the merits of the nonrelativistic multiple scattering - optical potential theory of nucleon-nucleus scattering. If significant failures in the description of the data occur then one may justifiably look to exotic physics mechanisms beyond that in traditional NR theories.

\section{Quasi-free $\Delta$ Production in Nuclei}

During the past few years a number of experiments have been conducted for quasi-free $\Delta$ production in nuclei by way of the charge exchange reactions $(p, n)$, $\left({ }^{3} \mathrm{He}, t\right)$ and $(d, 2 p)$. The data reveal a systematic downward energy shift of the $\Delta(1232 \mathrm{MeV})$ resonance in nuclei compared to that for free proton targets or as seen in inelastic electron scattering. It has been suggested that the longitudinal spin-isospin response, which can be excited with nucleons but not with electrons, is shifted downward in energy due to the strongly attractive delta particle - nucleon hole $\left(\Delta N^{-1}\right)$ effective interaction in the spin longitudinal channel at momentum 
transfers from $1-2 \mathrm{fm}^{-1}$.

Calculations indicate that this explanation is plausible. The key ingredients in the model are the $N N \rightarrow N \Delta$ transition potential and the $\Delta N^{-1}$ effective interaction in the nuclear medium. So far calculations have been based on simple $\pi$ and $\rho$ meson exchange models. During the forthcoming research period our NN coupled-channels isobar model will be used to generate the $N N \rightarrow N \Delta$ transition potential. The coupled-channels model of Ref. 6 includes $\pi$ exchange plus shortrange phenomenological terms for the $N N \rightarrow N \Delta$ excitation and provides an accurate description of the $N N$ isospin 1 inelasticities for all partial wave states through $J \leq 6$ for energies up to $1 \mathrm{GeV}$. The $N N \rightarrow N \Delta$ amplitude contains 16 independent, parity invariant terms. In addition to the spin transverse and longitudinal terms, preliminary calculations indicate that several of the rank-2 tensor amplitudes are large. The latter have not been included in quasifree $\Delta$ production calculations. We will improve the description of the $N N \rightarrow N \Delta, \Delta N^{-1}$ and $\Delta$-nucleus interactions and strive for a better understanding of quasi-free $\Delta$ production in nuclei. Calculation of spin observables will also be pursued.

\section{Studies of Nucleon-Nucleon Potentials Derived From Effective Chiral Lagrangians}

Recently Weinberg ${ }^{7}$ and collaborators ${ }^{8}$ have developed a chiral invariant effective Lagrangian for low rnomentum nucleons and pions. A static potential for interacting nucleons has been obtained in which the one-pion exchange potential (OPEP) is explicit. In addition to OPEP, spin-spin, spin-orbit, and tensor terms are also obtained. The potential contains a number of parameters representing higher mesonic degrees of freedom which have been integrated out of the effective Lagrangian. The parameters of the model will be fitted to low energy NN scattering phase shifts thereby yielding an effective, low energy $N \mathrm{~N} \pi$ Lagrangian, and a NN potential model for low energy in which the off-shell behavior is predicted by chiral symmetry. Use of this model in nuclear structure problems for light nuclei and in NN pion production will be considered. Inclusion of 1-loop pion diagrams and other meson exchanges can be added later.

\section{References}

[1] L. Ray, Phys. Rev. C 41, 2816 (1990).

[2] H. F. Arellano, F. A. Brieva and W. G. Love, Phys. Rev. C 41, 2188 (1990).

[3] Ch. Elster et al., Phys. Rev. C 41, 81'́1 (1990).

[4] G. Takeda and K. M. Watson, Phys. Rev. 97, 1336 (1955).

[5] A. Picklesimer and R. M. Thaler, Phys. Rev. C 23, 42 (1981). 
[6] L. Ray, Phys. Rev. C 35, 1072 (1987).

[7] S. Weinberg, Phys. Lett. B 251, 288 (1990); S. Weinberg, Univ. of Texas at Austin preprint, UTTG-03-91 (1991).

[8] C. Ordóñez and U. Van Kolck, private communication. 


\section{Publications (1990 - 1991)}

\subsection{Journal and Conference Proceedings in Print}

1. W. R. Coker and L. Ray, "The Nuclear Tensor Term in the Relativistic Impulse Approximation for Proton-Nucleus Elastic Scattering," Phys. Rev. C 42, 2242 (1990).

2. S. Shim, B. C. Clark, E. D. Cooper, S. Hama, R. L. Mercer, L. Ray, J. Raynal, and H. S. Sherif, "Comparison of Relativistic and Nonrelativistic Approaches to the Collective Model Treatment of $p+{ }^{40} \mathrm{Ca}$ Inelastic Scattering," Phys. Rev. C 42, 1592 (1990).

3. V. K. Mishra, S. Hama, B. C. Clark, R. E. Kozack, R. L. Mercer, and L. Ray, "Implications of Various Spin-One Relativistic Wave Equations for Intermediate Energy Deuteron-Nucleus Scattering," Phys. Rev. C 43, 801 (1991).

4. G. W. Hoffmann, M. L. Barlett, W. Kielhorn, G. Pauletta, M. Purcell, L. Ray, J. F. Amann, J. J. Jarmer, K. W. Jones, S. Penttilä, N. Tanaka, G. Burleson, J. Faucett, M. Gilani, G. Kyle, L. Stevens, A. M. Mack, D. Mihailidis, T. Averett, J. Comfort, J. Görgen, J. Tinsley, B. C. Clark, S. Hama, and R. L. Mercer, "Polarized Proton Elastic Scattering from Polarized ${ }^{13} \mathrm{C}$," Phys. Rev. Lett. 65, 3096 (1990).

5. M. L. Barlett, R. W. Fergerson, G. W. Hoffmann, J. A. Marshall, L. Ray, J. F. Amann, B. E. Bonner, and J. B. McClelland, "Inclusive Quasielastic Spin Observables for $\vec{p}+{ }^{2} \mathrm{H},{ }^{12} \mathrm{C}$ at $500 \mathrm{MeV}, "$ Phys. Lett. B 264, 21 (1991).

6. J. J. Görgen, J. R. Comfort, T. Averett, J. DeKorse, B. Franklin, B. G. Ritchie, J. Tinsley, G. Kyle, B. Berman, G. Burleson, K. Cranston, A. Klein, J. A. Faucett, J. J. Jarmer, J. N. Knudson, S. Penttilä, N. Tanaka, B. Brinkmöller, D. Dehnhard, Y. F. Yen, S. Hoibraten, H. Breuer, B. S. Flanders, M. A. Khandaker, D. L. Naples, D. Zhang, M. L. Barlett, G. W. Hoffmann, and M. Purcell, "Analyzing Powers for the Reaction $\pi^{-} \vec{p} \rightarrow \pi^{0} n$ at $T_{\pi^{-}}=161 \mathrm{MeV}$," Phys. Rev. D 42, 2374 (1990).

7. S. Penttilä, J. J. Jarmer, N. Tanaka, M. L. Barlett, K. Johnson, G. W. Hoffmann, W. Kielhorn, M. Purcell, G. Burleson, A. Klein, G. Kyle, B. Brinkmöller, D. Dehnhard, Yi-Fen Yen, J. R. Comfort, and J. J. Görgen, "LAMPF Polarized ${ }^{13} \mathrm{C}$ Targets," 9th International Symposium on High Energy Spin Physics, September 10-15, 1990, Physics, Institute, Bonn University, Bonn, Federal Republic of Germany, (Springer-Verlag, Berlin, Heidelberg, 1991), Vol. 2: Workshops, pp. 315-319. 
8. J. J. Görgen, J. R. Comfort, J. R. Tinsley, T. Averett, J. DeKorse, B. Franklin, B. G. Ritchie, G. Kyle, A. Klein, B. Berman, G. Burleson, K. Cranston, J. A. Faucett, J. J. Jarmer, J. N. Knudson, S. Penttilä, N. Tanaka, B. Brinkmöller, D. Dehnhard, Y. F. Yen, S. Hoibraten, H. Breuer, B. S. Flanders, M. A. Khandaker, D. L. Naples, D. Zhang, M. L. Barlett, G. W. Hoffmann, and M. Purcell, "Analyzing Powers for Pion Charge Exchange on Polarized ${ }^{13}$ C," Phys. Rev. Lett. 66, 2193 (1991).

9. D. M. Alde, H. W. Baer, T. A. Carey, G. T. Garvey, A. Klein, C. Lee, M. J. Leitch, J. Lillberg, P. L. McGaughey, C. S. Mishra, J. M. Moss, J. C. Peng, C. N. Brown, W. E. Cooper, Y. B. Hsiung, M. R. Adams, R. Guo, D. M. Kaplan, R. L McCarthy, G. Danner, M. Wang, M. Barlett, and G. Hoffmann, "A Dependence of $J / \psi$ and $\psi^{\prime}$ Production at $800 \mathrm{GeV} / \mathrm{c}$," Phys. Rev. Lett. 66, 133 (1991).

10. Yi-Fen Yen, B. Brinkmöller, D. Dehnhard, S. M. Sterbenz, Yi-Ju Yu, B. Berman, G. R. Burleson, K. Cranston, A. Klein, G. S. Kyle, R. Alarcon, T. Averett, J. R. Comfort, J. Görgen, B. Ritchie, J. R. Tinsley, M. Barlett, G. W. Hoffmann, K. Johnson, C. F. Moore, M. Purcell, H. Ward, A. Williams, J. A. Faucett, S. J. Greene, J. Jarmer, J. A. McGill, C. L. Morris, S. Penttilä, N. Tanaka, H. T. Fortune, E. Insko, R. Ivie, J. M. O'Donnell, D. Smith, M. A. Khandaker, and S. Chakravarti, "Asymmetry Measurement of Pion Elastic Scattering from Polarized ${ }^{13} \mathrm{C}$ in the Energy Region of the $P_{33}$ Resonance," Phys. Rev. Lett. 66, 1959 (1991).

11. G. W. Hoffmann, " $\vec{p}+{ }^{13} \overrightarrow{\mathrm{C}}$ Elastic cattering at $500 \mathrm{MeV}$, in Physics with Polarized Beams on Polarized Targets, edited by James Sowinski and Steven E. Vigdor, (World Scientific, Singapore, 1990), p. 139.

12. D. M. Alde, H. W. Baer, T. A. Carey, G. T. Garvey, A. Klein, C. Lee, M. J. Leitch, J. W. Lillberg, P. L. McGaughey, C. S. Mishra, J. M. Moss, J. C. Peng, C. N. Brown, W. E. Cooper, Y. B. Hsiung, M. R. Adams, R. Guo, D. M. Kaplan, R. L. McCarthy, G. Danner, M. J. Wang, M. L. Barlett, and G. W. Hoffmann, "Nuclear Dependence of the Production of $\Upsilon$ Resonances at $800 \mathrm{GeV}$, Phys. Rev. Lett. 66, 2285 (1991). 


\subsection{Journal Articles Submitted for Publication}

1. L. Ray, "Spin Dependent Effective Interaction for Medium Energy NucleonNucleus Scattering," in the Proceedings of the International Conference on Spin and Isospin in Nuclear Interactions, Telluride, CO, edited by S. Wissink, (Plenum, New York, 1991).

2. L. Ray, "Theoretical Interpretation of Proton Scattering Results from Polarized Nuclear Targets," in the proceedings of the 4th Conference on the Intersections Between Particle and Nuclear Physics, Tucson, AZ, (American Institute of Physics, New York, 1991).

3. L. Ray, "Nuclear Structure and Medium Effects in $\vec{p}+{ }^{13} \overrightarrow{\mathrm{C}}$ Elastic Scattering," submitted to Phys. Rev. C (1991).

4. L. Ray, G. W. Hoffmann and W. R. Coker, "Relativistic and Nonrelativistic Descriptions of Proton-Nucleus Scattering," Physics Reports (1990), in press:

\subsection{EXP791/871 KL-Memos (Internal Publications)}

1. J. McDonough and M. Diwan, "Results of Beam-Plug Analysis," KL-297 (1990).

2. G. Hoffmann, J. McDonough, B. Ware and S. Worm, "Measurements of Neutron and Photon Flux from the Beam Plug," KL-337 (1991).

3. B. Ware, G. Hoffmann, J. McDonough, and S. Worm, "Bonner Spheres/BUNKI and ${ }^{3} \mathrm{He}$ Counter Measurements," KL-338 (1991).

4. S. D. Worm, G. W. Hoffmann, J. McDonough, and C. B. Ware, "Analysis of the Liquid Scintillator Data Taken During EXP871," KL-339 (1991).

5. J. McDonough, G. Hoffmann, B. Ware, and S. Worm, "Measurements of Photons from the Beam Plug," KL-340 (1991).

6. J. McDonough, G. Hoffmann, B. Ware, and S. Worm, "Measurements of Charged Particle Flux from the Beam Plug," KL-341 (1991).

7. G. Hoffmann, J. McDonough, B. Ware, and S. Worm, "Estimates of EXP871 Drift Chamber Rates from Texas Detector Measurements," KL-345 (1991). 


\subsection{Abstracts}

1. L. Ray, G. W. Hoffmann and W. R. Coker, "Nonrelativistic and Relativistic Descriptions of Proton-Nucleus Scattering," Texas Section Meeting of the American Physical Society, Denton, Texas, Nov. 1-2, 1991, Bull. Am. Phys. Soc. (1991).

2. D. Read, A. Green, G. Hoffmann, L. Ray, S. Worm, and J. Amann, "A High Precision Charged Particle Beam Profile Monitor," Texas Section Meeting of the American Physical Society, Denton, Texas, Nov. 1-2, 1991, Bull. Am. Phys. Soc. (1991).

3. J. McDonough, G. Hoffmann, K. Lang, P. Riley, J. Ritchie, B. Ware, and S. Worm, "Beam-Plug Design for BNL EXP871," Texas Section Meeting of the American Physical Society, Denton, Texas, Nov. 1-2, 1991, Bull. Am. Phys. Soc. (1991).

4. B. Ware, G. Hoffmann, K. Lang, J. McDonough, P. Riley, J. Ritchie, and S. Worm, "Measurement of Neutrons from a Neutron Beam Stop," Texas Section Meeting of the American Physical Society, Denton, Texas, Nov. 1-2, 1991, Bull. Am. Phys. Soc. (1991).

5. S. Worm, G. Hoffmann, K. Lang, J. McDonough, P. Riley, J. Ritchie, and B. Ware, "Measurement of Photons and Charged Particles from a Neutral Beam Stop," Texas Section Meeting of the American Physical Society, Denton, Texas, Nov. 1-2, 1991, Bull. Am. Phys. Soc. (1991). 

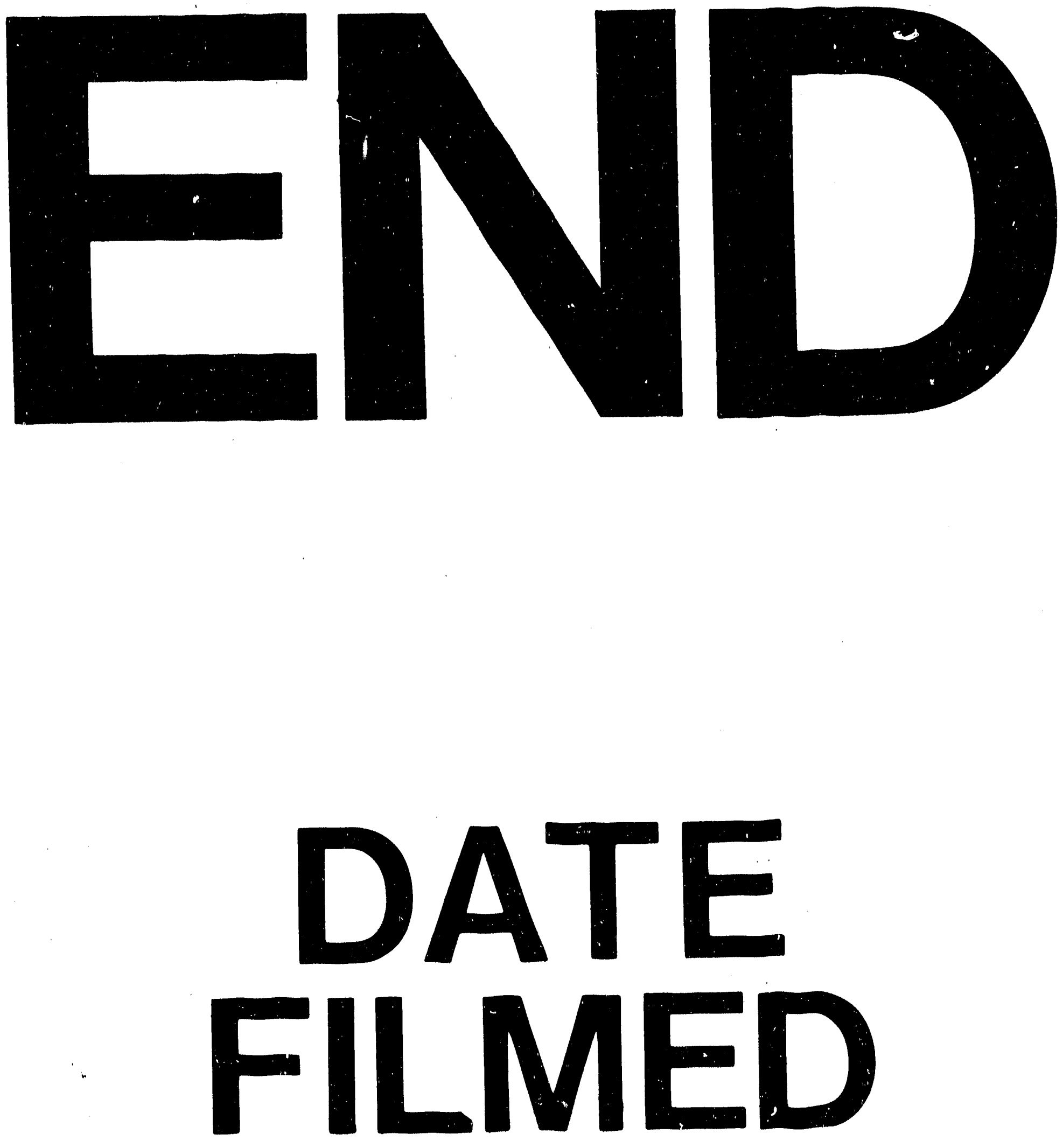

1

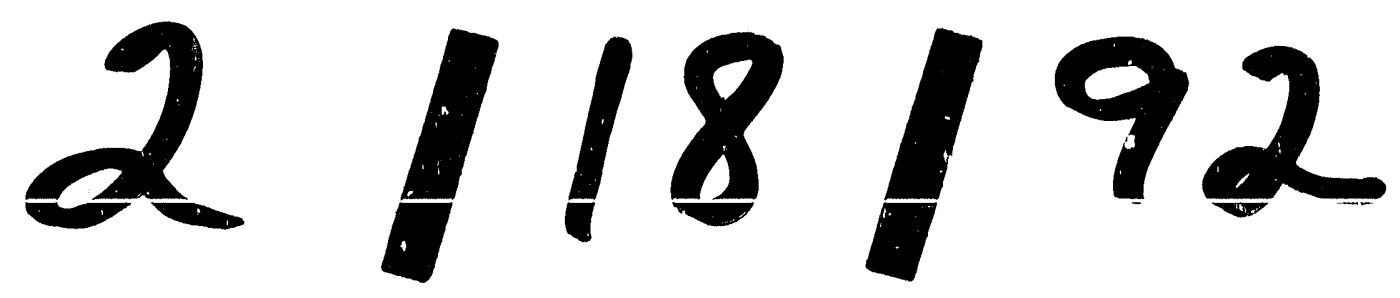

